Aus der Abteilung Präventive Zahnmedizin, Parodontologie und Kariologie

(komm. Direktor: Prof. Dr. med. dent. M. Hülsmann) im Zentrum Zahn-, Mund- und Kieferheilkunde der Medizinischen Fakultät der Universität Göttingen

\title{
Überlebens- und Erfolgsrate von Keramik-Veneers nach 3 Jahren Tragezeit
}

\author{
INAUGURAL-DISSERTATION \\ zur Erlangung des Doktorgrades \\ für Zahnheilkunde \\ der Medizinischen Fakultät \\ der Georg-August-Universität zu Göttingen
}

vorgelegt von

Julian Spellmann

aus

Göttingen

Göttingen 2011 
Dekan: Prof. Dr. med. C. Frömmel

I. Berichterstatter: Prof. Dr. med. dent. R. F. Mausberg II. Berichterstatter: Prof. Dr. med. dent. M. Hülsmann III. Berichterstatter/in:

Tag der mündlichen Prüfung: 12.12.2011 


\section{Gliederung}

1 Einleitung 1

2 Literaturübersicht 4

2.1 Ästhetische Korrekturen der Zähne 4

2.1.1 Allgemeines 4

2.1.2 Materialien 5

2.1.2.1 Keramik 5

2.1.2.2 Dentalkeramik 6

2.1.2.2.1 Abgrenzung der Keramik von Porzellan 6

2.1.2.2.2 Zusammensetzung und Materialeigenschaften $\quad 7$

2.1.3 Herstellung eines Verbunds zwischen Zahn und Keramik (SÄT) 10

2.1.3.1 Konditionierung der Keramikoberfläche 11

2.1.3.2 Konditionierung der Zahnoberfläche 11

2.1.3.3 Befestigungsmöglichkeiten 13

2.1.3.4 Adhäsionskomplex Zahn-Zement-Keramik 13

$\begin{array}{ll}2.1 .4 \text { Versorgungsarten } & 13\end{array}$

$\begin{array}{ll}\text { 2.1.4.1 Veneer } & 14\end{array}$

2.1.4.1.1 Geschichte der Veneer-Technik $\quad 15$

2.1.4.1.2 Indikationen und Kontraindikationen 16

2.1.4.1.2.1 Indikationen 16

2.1.4.1.2.2 Kontraindikationen 18

2.1.4.1.3 Vorteile und Nachteile $\quad 19$

$\begin{array}{ll}\text { 2.1.4.1.3.1 Vorteile } & 19\end{array}$

2.1.4.1.3.2 Nachteile 20

2.1.4.1.4 Präparationsformen $\quad 21$

2.1.4.1.5 Klassifizierung der Keramik-Systeme für Veneers 24

2.1.4.1.6 Erfolgskriterien und -aussichten 26

2.1.4.1.6.1 Anforderungen 26

2.1.4.1.6.2 Überlebens-und Erfolgsrate 28

3 Material und Methoden $\quad 30$

3.1 Administrative Vorbereitungen 30

$\begin{array}{ll}3.2 \text { Material } & 30\end{array}$

$\begin{array}{ll}3.2 .1 \text { Probanden } & 30\end{array}$

3.2.1.1 Probandenverwaltung und -dokumentation 30

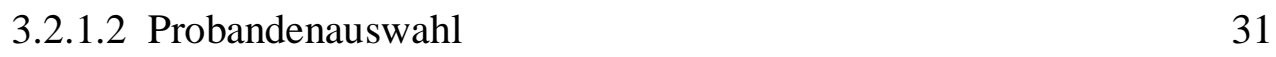

3.2.1.2.1 Einschlusskriterien $\quad 31$

3.2.1.2.2 Ausschlusskriterien $\quad 32$

$\begin{array}{ll}3.2 .2 \text { Veneers } & 32\end{array}$

3.2.2.1 Präparation $\quad 32$

3.2.2.2 Keramik 33

3.2.2.3 Adhäsivsystem und Befestigungszement 34

3.2.2.3.1 Adhäsivsystem 34

$\begin{array}{ll}\text { 3.2.2.3.2 Befestigungszement } & 34\end{array}$ 
3.2.2.3.2.1 Calibra $\quad 35$

3.2.2.3.2.2 Variolink 35

3.2.2.3.3 Indikationen und Kontraindikationen 36

3.2.2.3.4 Arbeitsschritte 36

$\begin{array}{ll}3.3 \text { Methoden } & 37\end{array}$

3.3.1 Klinische Untersuchungen 37

3.3.2 Statistische Auswertung der Ergebnisse 38

4 Ergebnisse $\quad \mathbf{4 0}$

4.1 Probanden 40

4.2 Veneers 43

4.3 Verlust- und Erfolgsrate $\quad 45$

$\begin{array}{ll}\text { 4.3.1 Verlustrate } & 45\end{array}$

4.3.1.1 Faktor Zement $\quad 48$

4.3.1.2 Faktor Region $\quad 49$

4.3.1.3 Schätzung der Überlebensrate $\quad 50$

$\begin{array}{ll}\text { 4.3.2 Erfolgsrate } & 51\end{array}$

4.3.2.1 Faktor Zement $\quad 53$

4.3.2.2 Faktor Region $\quad 55$

4.3.2.3 Schätzung der Erfolgsrate $\quad 56$

4.3.3 Endergebnisse $\quad 57$

5 Diskussion $\quad \mathbf{5 9}$

5.1 Material $\quad 59$

5.1.1 Probandenauswahl 59

5.1.2 Bewertung der Präparation, des Adhäsivsystems und der Zemente 60

5.1.3 Bewertung der verwendeten Presskeramik 61

5.2 Methodik 62

5.2.1 Problematik von in-vivo-Studien 62

5.2.2 Beurteilung der Untersuchungsmethodik 63

5.3 Ergebnisse $\quad 64$

5.4 Schlussfolgerung $\quad 68$

6 Zusammenfassung $\quad 69$

7 Literaturverzeichnis $\quad 70$

8 Anhang $\quad 84$

$\begin{array}{lll}\text { 8.1 USPHS-Kriterien } & 84\end{array}$

8.2 Genehmigung des Ethikantrags 85

$\begin{array}{lll}8.3 & \text { Untersuchungsbogen } & 86\end{array}$ 


\section{Einleitung}

Ästhetische Zahnmedizin gewinnt zunehmend an Bedeutung.

Es gibt eine Vielzahl von Möglichkeiten, um den hohen Ansprüchen gerecht zu werden. Hierzu zählen Inlays, Teilkronen, Kronen, die kieferorthopädische Behandlung, das Bleichen und die Versorgung mittels eines Veneers. Seit über 20 Jahren haben die adhäsiv befestigten Veneers im Frontzahnbereich einen großen Anteil an der ästhetischen Zahnheilkunde (SWIFT und FRIEDMAN 2006). In mehreren Studien haben sie sich trotz minimal invasiver Maßnahmen als stabile, resistente und haltbare Restaurationen erwiesen (KREULEN et al. 1998, PEUMANS et al. 2000, DELLA BONA und KELLY 2008). In retrospektiven Langzeitstudien von bis zu 15 Jahren erzielten Veneers eine Überlebensrate von 93\% (FRIEDMAN 1998). Die Misserfolgsrate lag laut einer Literaturübersicht aus dem Jahr 2000 zwischen null und maximal fünf Prozent bei Beobachtungszeiträumen von einem bis fünf Jahre (PEUMANS et al. 2000). Als Einflussfaktoren für die Überlebensrate wurden Variablen wie statische und dynamische Okklusion, Präparationsdesign/-typ, Vorhandensein von Kompositfüllungen, Dentinfreilegung und das verwendete Adhäsivsystem beschrieben (EDELHOFF und ÖZCAN 2007). In der Mehrzahl der Studien werden die untersuchten Veneers aus Feldspatkeramik hergestellt (CHEN et al. 2005, LAYTON und WALTON 2007, PEUMANS et al. 2004, SHAINI et al. 1997, FRIEDMAN 1998, DUMFAHRT und SCHAFFER 2000, BURKE und LUCAROTTI 2009). Darüber hinaus liegen mehrere Untersuchungen zu Veneers aus Presskeramik vor, die sich im Vergleich zu Feldspatkeramiken durch bessere mechanische Eigenschaften auszeichnet (GUESS und STAPPERT 2008, ARISTIDIS und DIMITRA 2002, FRADEANI 1998, AYKOR und OZEL 2009). Alle Studien beschreiben eine Überlebensrate zwischen 96,5\% und 98,8\% nach viereinhalb bis sechs Jahren Beobachtung (ARISTIDIS und DIMITRA 2002, FRADEANI 1998).

In den letzten Jahren hat sich das Einsatzspektrum von keramischen Materialien stetig erweitert, weil sich die mechanischen Eigenschaften der Keramik und der Klebeverbund der Befestigungskomponenten immer stärker verbessert haben. Heutzutage können Veneers nicht nur zur Versorgung verfärbter oder deformierter/missgebildeter Zähne im Frontzahngebiet eingesetzt werden, sondern aufgrund weiterentwickelter Präparationstechniken auch zunehmend bei schwereren Defekten 
morphologischer oder struktureller Art. Dies belegt zum Beispiel die 2008 erschienene Studie von GUESS und STAPPERT, bei der nach fünf Jahren die Überlebensrate von Presskeramik-Veneers 97,5\% betrug. Auch hier wurden großflächig extendierte Overlap-Veneers untersucht, was deren gute Verwendungsmöglichkeiten auch bei großen Defekten belegt. Aufgrund der Ergebnisse vieler klinischer Studien, in denen Siliziumoxidkeramiken untersucht wurden, sollten Veneers adhäsiv mit Komposit zementiert werden (KREULEN et al. 1998, PEUMANS et al. 2000, DELLA BONA und KELLY 2008, EDELHOFF und ÖZCAN 2007).

Jedoch fehlen bis heute Untersuchungen, die den Einfluss von verschiedenen Kompositzementen auf die Erfolgs- und Überlebensrate von Keramik-Veneers in Langzeitstudien untersuchen. Obwohl sich die Technik zur Versorgung der Zähne mit Veneers nicht für Ober- und Unterkiefer unterscheidet, ist die Anzahl der Studien, in denen Unterkieferveneers untersucht wurden, begrenzt. In den meisten vorliegenden Studien war bisher nicht zwischen Ober- und Unterkiefer unterschieden und nur die Summe an Veneers betrachtet worden, da häufig nur sehr wenig Veneers im Unterkiefer positioniert waren. Somit wurde bisher der Einfluss der Kieferposition des Veneers auf die Erfolgs- und Überlebensrate vernachlässigt, wodurch keine Differenzierung der Überlebensraten in Abhängigkeit von der Zeit möglich waren (MURPHY et al. 2005, GUESS und STAPPERT 2008, BURKE und LUCAROTTI 2009, LAYTON und WALTON 2007, PEUMANS et al. 2000, PEUMANS et al. 2004).

Aus klinischer Sicht ist die erfolgreiche Versorgung von Frontzähnen im Unterkiefer mit Keramik-Veneers eine der anspruchsvollsten Restaurationsarten in der ästhetischen Zahnheilkunde. Faktoren, die zu Misserfolgen im Unterkiefer führen können, sind die kleine klinische Krone, eine geringe Schmelzdicke, die für eine adhäsive Befestigung essentiell ist (v.a. zervikal), Befestigungsmaterialien, Belastungskräfte, die auf den Zahn-Veneer-Komplex einwirken und die funktionelle und parafunktionelle Okklusion (WALTER und RAIGRODSKI 2008). Aus diesen Gründen kann bisher keine klare Aussage über die klinische Überlebensrate von Unterkiefer-Veneers im Vergleich zu Oberkiefer-Versorgungen gemacht werden. Klinische Studien mit einer großen Anzahl von Unterkiefer-Restaurationen mit Keramik-Veneers, die nach den heutigen Standards adhäsiv befestigt wurden, sind geeignet, klinische Entscheidungshilfen zu entwickeln (WALTER und RAIGRODSKI 2008).

Vor diesem Hintergrund ist es das Ziel der vorliegenden Studie, die Überlebens- und Erfolgsrate von Veneers mit Overlap-Präparation im Ober- und Unterkiefer zu unter- 
suchen, die aus Presskeramik hergestellt wurden. Die retrospektive Bewertung der klinischen Beständigkeit bezieht sich auf eine Tragezeit von 36 Monaten.

Die Arbeitshypothese lautet: Es gibt keinen Einfluss der Kieferposition des Veneers und des Befestigungszements auf ihre Erfolgs- und Überlebensrate. 


\section{Literaturübersicht}

\section{1 Ästhetische Korrekturen der Zähne}

\subsubsection{Allgemeines}

Ästhetik spielt im heutigen Leben vieler Menschen eine große und wichtige Rolle. Sie wird durch die Medien in den Mittelpunkt des gesellschaftlichen Empfindens gestellt. Für DIEDRICH (1995) ist sie sogar entscheidend für die Lebensqualität jedes Einzelnen. Eine besondere Bedeutung kommt dabei dem Gesichtsausdruck und der Mundpartie zu. „Ein harmonisch balanciertes Lächeln entsteht im Zusammenspiel von dentaler und gingivaler Ästhetik. Ästhetische Defizite der Gingiva können auch mit dem perfektesten Zahnersatz nicht kompensiert werden - dasselbe gilt auch umgekehrt“ (MAGNE und BELSER 2004, S. 58).

BELSER (1982) stellte eine Bewertungsliste auf, in der Kriterien in Bezug auf die Ästhetik der harten und weichen Gewebestrukturen beschrieben werden. Sie wurde von MAGNE und BELSER (2004) noch etwas abgeändert und enthält folgende Elemente:

Objektive Kriterien:

1. Zustand der Gingiva

2. Geschlossene Interdentalräume

3. Zahnachse

4. Höchster Punkt des Gingivasaums

5. Balancierter Verlauf der Gingiva

6. Höhe des interdentalen Kontaktpunkts

7. Dentale Größenverhältnisse

8. Hauptmerkmale der Zahnform

9. Charakterisierung der Zähne

10. Oberflächenstruktur

11. Farbe

12. Schneidekante

13. Linienverlauf der Unterlippe

14. Symmetrie des Lächelns. 
Subjektive Kriterien (ästhetische Integration):

1. Unregelmäßige Zahnformen

2. Anordnung und Stellung der Zähne

3. Relative Kronenlängen

4. Schwarzer Raum der Mundhöhle.

Für den Erfolg von Zahnkorrekturen ist eine Harmonie zwischen den objektiven und den subjektiven Kriterien notwendig, um die Ästhetik aus zahnmedizinischer Sicht mit der für jeden Patienten individuellen und charakteristischen Ästhetik in Einklang zu bringen (MAGNE et al. 1993). Laut MAGNE et al. (1996) ist die Gesamtharmonie subjektiv und davon abhängig, wie gut diese Parameter in das Lächeln, die Gesichtsform, das Alter und den Charakter des Patienten integriert werden.

\subsubsection{Materialien}

Wie schon zum Teil oben erwähnt, können Inlays, Teilkronen und Kronen aus Komposit, Metall, Metall-Keramik oder Keramik bestehen. Da die in dieser Studie untersuchten Veneers jedoch ausschließlich aus Keramik hergestellt wurden, soll im weiteren Verlauf lediglich auf dieses Material eingegangen werden.

\subsubsection{Keramik}

Die Entwicklung der Vollkeramik wurde vor allem durch den stark ansteigenden Stellenwert der Ästhetik und durch die Sensibilisierung für allergische Reaktionen auf metallischen Zahnersatz vorangetrieben (KAPPERT et al. 1991). Die Indikationsbreite ist hinsichtlich der mechanischen Festigkeit herkömmlicher Dentalkeramiken eingeschränkt, da diese den hohen Kaukräften nicht standhält. Dieser Aspekt wird durch die grazile Gestaltung aus funktionellen und ästhetischen Gründen noch verstärkt. Dies treibt die Forschung vor allem im Bezug auf die Festigkeitssteigerung der vollkeramischen Werkstoffe an (EVANS und O`BRIEN 1999).

Auf die Dentalkeramik wird im weiteren Verlauf noch ausführlich eingegangen. 


\subsubsection{Dentalkeramik}

\subsection{Abgrenzung der Keramik von Porzellan}

Das Porzellan und die Dentalkeramik haben als gemeinsame Bestandteile Feldspat, Kaolin und Quarz (ROSENBLUM und SCHULMAN 1997). Lediglich die Anteile dieser Komponenten sind aufgrund der verschiedenen Anforderung und Aufgaben an diese beiden Werkstoffe unterschiedlich (EICHNER 1985, POß 1983).

Betrachtet man die Dentalkeramik, so erkennt man die Erhöhung des Gewichtsanteils des Feldspats, die eine Reduktion des Kaolinanteils zufolge hat. Das Ausmaß dieser Abweichungen ist aus der unterschiedlichen Lage von Keramik und Porzellan im Dreistoffsystem Kaolin-Feldspat-Quarz erkennbar (s. Abb.1) (BARREIRO et al. 1989, CLAUS 1980, SALMANG und SCHOLZE 1983).

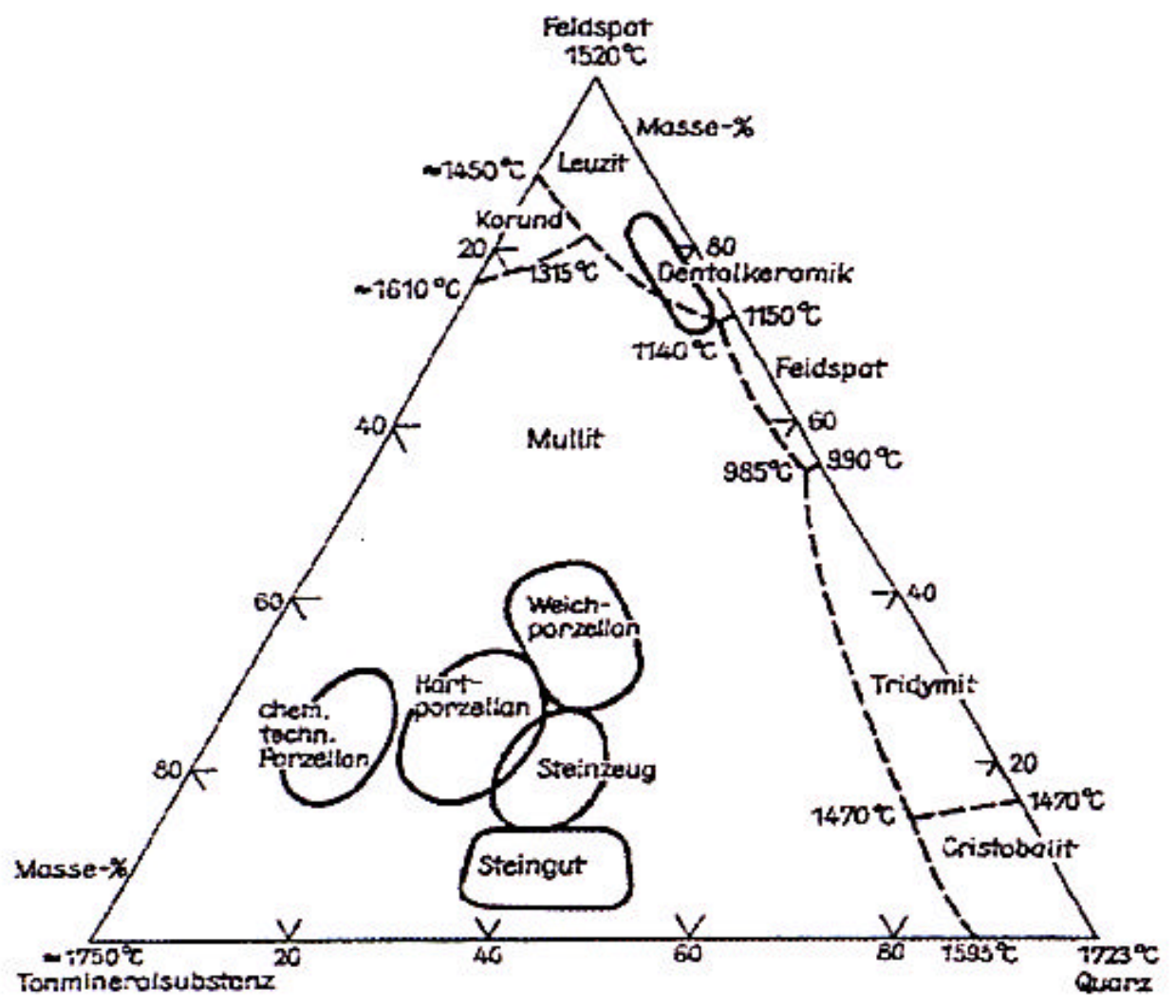

Abb.1: Phasendiagramm für Feldspat-Quarz-Kaolin (CLAUS 1980, S. 1748) 
Dieses Verhältnis bei der Dentalkeramik, viel Feldspat und wenig Kaolin, bewirkt den glasähnlichen Charakter, wodurch sie sich vom Porzellan unterscheidet, was die Tabelle 1 verdeutlicht.

Des Weiteren wird das Ausscheidungsprodukt als Unterscheidungsmerkmal gesehen. Bei der Dentalkeramik sind dies Leuzitkristalle $\left(\mathrm{K}_{2} \mathrm{O} \mathrm{Al} \mathrm{O}_{3} 4 \mathrm{SiO}_{2}\right)(\mathrm{MORENA}$ et al. 1986, POß 1983), beim Porzellan sind es Mullitkristalle $\left(3 \mathrm{Al}_{2} \mathrm{O}_{3} 2 \mathrm{SiO}_{2}\right)(\mathrm{BARREI}-$ RO et al. 1989, CLAUS 1980, FREISBERG 1985, GREMMINGER 1997, SALMANG und SCHOLZE 1983).

Tab.1: Gegenüberstellung der abweichenden Zusammensetzung von Porzellan und Dentalkeramik CLAUS (1980)

\begin{tabular}{|c|c|c|c|}
\hline & Feldspat & Quarz & Kaolin \\
\hline Dentalkeramik & $70-80 \%$ & $10-30 \%$ & $0-3 \%$ \\
\hline Porzellan & $10-30 \%$ & $15-35 \%$ & $40-70 \%$ \\
\hline
\end{tabular}

\subsection{Zusammensetzung und Materialeigenschaften}

Als Keramik bezeichnete man lange Zeit die Materialien, die aus tonhaltigen Rohstoffen gewonnen wurden. Heute ist die Definition eine andere. Keramik ist als anorganisch-nichtmetallischer, weitgehend kristalliner bzw. ganzkristalliner Körper zu bezeichnen, der durch den Vorgang des Brennens (Sintern) vom pulvrigen in den festen Zustand überführt wird (BREUSTEDT und LENZ 1978, MARX 1993). Diese kristalline Struktur macht den Unterschied zu amorphen (strukturlosen) Gläsern aus (BREUSTEDT und LENZ 1978, TIETZ 1994, SCHNAPP und KÖHLER 1990).

Keramische Werkstoffe bestehen zumeist aus unterschiedlichen Kristallen und sind somit heterogene Strukturen. Diese sind von einer Glasphase umgeben. Ionen- und Atombindungen sorgen für die chemische Bindung. Die Eigenschaften der Keramikmasse sind abhängig von der Struktur. Die Atome lagern sich in einer Kristallgitterstruktur an, in der die Atome sich zu Gruppen geordnet haben. Amorphes Glas entsteht bei der schnellen Abkühlung der Siliziumdioxidschmelze, was eine geordnete Anordnung der Atomgruppen verhindert. Kühlt die Masse langsam ab, so entsteht wieder das oben beschrieben Gitter der $\mathrm{SiO}_{2}$-Moleküle. Es bilden sich Kristalle. Ver- 
antwortlich für die große Härte und den hohen Schmelzpunkt der Keramik sind die atomaren Bindungen. Da die Atome im Gitter sehr dicht aneinander liegen, ist die Festigkeit sehr hoch im Gegensatz zu amorphem Glas, das bruchgefährdeter ist (BOWEN 1986, CAMPBELL 1989, CLAUS 1990, SCHNAPP und KÖHLER 1990).

Wie schon oben beschrieben, stehen die Entwicklung des Porzellans und die der Dentalkeramik in einem engen Zusammenhang.

Die Zusammensetzung dieser beiden Werkstoffe unterscheidet sich vor allem darin, dass in der heutigen Dentalkeramik so gut wie kein Kaolin mehr vorhanden ist, im Porzellan hingegen bis zu 70\%. Aus diesem Grund liegt bei der Dentalkeramik auch kein Multikristallgefüge vor.

Dentalkeramiken stehen in Bezug auf ihre Zusammensetzung zwischen Hartporzellan und Gläsern (BREUSTEDT und LENZ 1978, TIETZ 1994).

Feldspat ist ein Mineral, chemisch gesehen ein Silikat. Als Silikate bezeichnet man die Verbindungen von Kieselsäure (Quarz) mit bestimmten Basenbildnern wie Kalium, Natrium, Eisen und Aluminiumoxid (EICHNER 1985, GREMMINGER 1997). Dabei spielt die Anzahl der Basen keine Rolle.

Feldspat stellt die Glasphase dar, in die Quarz eingelagert ist (EICHNER 1985).

Man teilt die Feldspate in vier Gruppen ein (BROSTOW 1984):

- Kalifeldspat (Orthoklas genannt) mit der Formel $\left(\mathrm{K}_{2} \mathrm{O} \mathrm{Al}_{2} \mathrm{O}_{3} 6 \mathrm{SiO}_{2}\right)$

- Natronfeldspat (Albit genannt) mit der Formel $\left(\mathrm{Na}_{2} \mathrm{O} \mathrm{Al}_{2} \mathrm{O}_{3} 6 \mathrm{SiO}_{2}\right)$

- Kalkfeldspat (Anorthit genannt) mit der Formel $\left(\mathrm{CaO} \mathrm{Al}_{2} \mathrm{O}_{3} 6 \mathrm{SiO}_{2}\right)$

- Lithiumfeldspat (Spodumen genannt) mit der Formel $\left(\mathrm{Li}_{2} \mathrm{O} \mathrm{Al} \mathrm{Al}_{3} \mathrm{O}_{3} 6 \mathrm{SiO}_{2}\right)$.

Von größter Bedeutung für die Dentalkeramik ist der Kalifeldspat (FREISBERG 1985). Aufgrund der Tatsache, dass Orthoklas inkongruent schmilzt, zerfällt er beim Schmelzvorgang in Schmelze und Leuzit (CLAUS 1980, KON et al. 2001, LINDEMANN 2000, SCHMID et al. 1992).

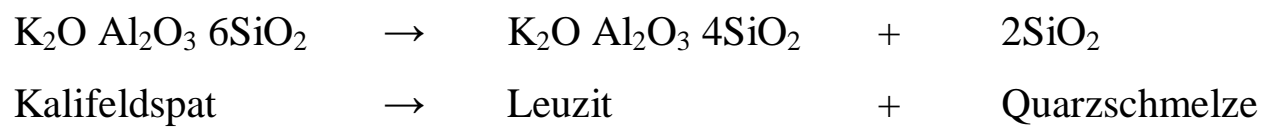


Leuzit bleibt von nun an metastabil im Glas erhalten, da bei der Abkühlung keine Rekristallisation in Kalifeldspat erfolgt. Der Gehalt an Leuzit hängt somit von dem Kalifeldspatgehalt, der Temperatur und der Dauer der Wärmebehandlung ab.

Der Leuzitgehalt ist vor allem für den Wärmeausdehnungskoeffizienten und die mechanische Festigkeit von großer Bedeutung (CLAUS 1980, KELLY et al. 1996, KON et al. 2001, LINDEMANN 2000, MACKERT und WILLIAMS 1996).

Wie oben schon gesagt, kommt es während der Abkühlung der Leuzitkristalle zur Schrumpfung. Daraus folgt wiederum eine Festigkeitssteigerung. Diese Kontraktion bewirkt eine Druckspannung in der Glasmatrix, aus der wiederum eine Risswegverlängerung resultiert. D. h., dass Risse von den Leuzitkristallen aufgefangen oder reflektiert werden (HÖLSCH und KAPPERT 1992).

Neben diesen wichtigen Effekten der Leuzitkristalle gibt es noch andere wichtige Eigenschaften. Sie steigern die Opazität der Glasphase, die teils angestrebt ist und teils unerwünscht ist (im Bezug auf Ästhetik) (GREMMINGER 1997, LUDWIG 1991). Des Weiteren spielt die Steigerung der Kantenfestigkeit der Restauration, Viskosität und Standfestigkeit der Schmelze im Bezug auf die Formstabilität eine große Rolle (CLAUS 1980, SCHULTHEISS 1992).

Feldspat besitzt ein niedriges Schmelzintervall. Dieses schwankt je nach Typ zwischen $1150^{\circ} \mathrm{C}$ und $1500^{\circ} \mathrm{C}$. Bei großer Hitze lösen sich große Mengen an Quarz und Kaolin. Aufgrund dieser Tatsache dient Feldspat als Flussmittel. Feldspat, der bereits schon einmal geschmolzen wurde und wieder abgekühlt ist, schmilzt beim nächsten Mal bei einer niedrigeren Temperatur. Daher schmilzt eine vorgebrannte und feldspatreiche Dentalkeramik schneller als eine unbehandelte Keramik (FREISBERG 1985).

Quarz (Kieselsäure) ist ein Siliziumdioxid $\left(\mathrm{SiO}_{2}\right)$, das in zahlreichen Modifikationen vorliegen kann. Er ist das auf der Erde am meisten vorkommende Material. Die $\mathrm{SiO}_{2}$-Moleküle bewirken die Ausbildung eines dreidimensionalen Netzwerks, wobei ein Siliziumatom tetraedisch von vier Sauerstoffatomen umgeben ist (LINDEMANN 2000).

Gesteine, in denen Quarz vorkommt, sind unter anderem Granit, Gneis und Quarzporphyr. Für die Keramik sind vor allem von Bedeutung der Stückquarz, der Rosenquarz, der Quarzsand und der Quarzit. Diese müssen eine hohe Reinheit aufweisen, 
da ansonsten die Keramik Verfärbungen aufweist (BROSTOW 1984, EICHNER 1985, LINDEMANN 2000).

Quarz hat bei der Dentalkeramik die Aufgabe der Festigkeitssteigerung und der Transparenzerhöhung. Metalloxide können die Farbe der Keramik variieren und werden daher je nach gewünschter Helligkeit beigemischt. Der Begriff „Magerungsmittel“, wie Quarz in der Fachsprache auch genannt wird, kommt daher, dass Quarz die Schwindung der Keramik durch das thermovolumetrische Verhalten verringert. Der Schmelzpunkt liegt zwischen $1400^{\circ} \mathrm{C}$ und $1600^{\circ} \mathrm{C}$ (GREMMINGER 1997, SCHULTHEISS 1992).

Kaolin (Tonerde) wird auch Porzellanton oder Chinaclay genannt.

Wie schon erwähnt, spielt dieser Bestandteil in der Dentalkeramik eine geringe Rolle, da er nur in Spuren vorhanden ist. Kaolin entsteht bei der Verwitterung der primären Erstarrungsgesteine, v.a. aus Granit, Quarzporphyr und Gneis, und wird somit als Gestein sekundärer Bildung bezeichnet.

Aus chemischer Sicht entspricht Kaolin einem Alumosilikat $\left(\begin{array}{llll}\mathrm{Al}_{2} \mathrm{O}_{3} & 2 \mathrm{SiO}_{2} & \mathrm{H}_{2} \mathrm{O}_{2}\end{array}\right)$ (EICHNER 1985, FREISBERG 1985, LINDEMANN 2000).

Das Wasser ist in Form von Hydroxylgruppen gebunden. Die Schmelztemperatur liegt bei $1780^{\circ} \mathrm{C}$, wobei die Sinterung schon bei $1600^{\circ} \mathrm{C}$ eintritt.

Auch Kaolin hat einen großen Einfluss auf die Formbeständigkeit der Keramik beim Brand. Dies kommt daher, dass bei Zugabe von Wasser zu Kaolin eine plastische Masse entsteht, die geformt werden kann (SCHULTHEISS 1992).

Durch Tonerde wird auch die Opazität beeinflusst (FREISBERG 1985).

\subsubsection{Herstellung eines Verbunds zwischen Zahn und Keramik (SÄT)}

Ein wichtiger Faktor für die Langlebigkeit eines Veneers ist der Adhäsionskomplex. Dieser wird gebildet vom Veneer, dem Bonding und dem Zahn. Je stärker er ist, desto höher ist die Wahrscheinlichkeit, dass das Veneer lange im Mund verbleibt. 


\subsubsection{Konditionierung der Keramikoberfläche}

Laut JARDEL et al. (1999) ist für den Klebeverbund seitens der Keramik eine mikromechanische Verankerung mittels einer Fluorwasserstoffsäure (Flusssäure) sowie eine Silanisierung als chemischer Verbund nötig.

Die Ätzung mit Flusssäure auf der Veneerinnenseite bewirkt ein retentives amorphes Ätzmuster, welches sich durch zahlreiche Porositäten auszeichnet (PEUMANS et al. 1999). In diese aufgeraute Oberfläche dringt das Bonding ein und verzahnt sich mit den Mikroretentionen bei der Aushärtung. Es gibt diverse Faktoren, die das Ätzmuster und somit den Haftverbund zwischen Restauration und Zahn beeinflussen. Diese sind die Ätzdauer, die Säurekonzentration, die Keramikherstellungsweise (SIMONSEN und CALAMIA 1983, STANGEL et al. 1987) und die Keramikbeschaffenheit (CALAMIA 1985, ROULET et al. 1995). Nach der Ätzung sollte die Facette mit 95\%igem Alkohol, Aceton oder destilliertem Wasser im Ultraschallbad gereinigt werden, um Säurereste und Keramikpartikel, die durch das Ätzen herausgelöst wurden, zu entfernen (PEUMANS et al. 2000).

Nach der Ätzung wird die Keramikoberfläche silanisiert, um so einen chemischen Verbund zum Bonding zu ermöglichen. Dies geschieht mit einem bifunktionellen Kopplungsmittel welches zum einen aus einer Silangruppe, die sich mit dem hydrolysierten Silikondioxid auf der Keramikoberfläche chemisch verbindet, besteht. Zum anderen polymerisiert sich die Methacrylatgruppe an die Adhäsivschicht (PEUMANS et al. 2000). Negativen Einfluss auf die Haftkraft haben die Kontamination der Oberflächen mit Schleifresten (SWIFT et al. 1995), sowie Latexhandschuhe (HOLTAN et al. 1995), Speichel (NICHOLLS 1988), silikonbasierte Fit-checkerPaste (SHETH et al. 1988) und die Anprobepaste (try-in-paste) (DELLA BONA und NORTHEAST 1994).

\subsubsection{Konditionierung der Zahnoberfläche}

Die Konditionierung der Oberfläche hängt von der klinischen Situation ab.

Liegt die gesamte Präparation im Schmelz, muss anders vorgegangen werden, als wenn man das Dentin mit eröffnen würde. 


\section{Schmelzbegrenzte Präparation:}

Der Schmelz wird, wie oben beschrieben, reduziert, um den Haftverbund zu verstärken (STACEY 1993), indem die aprismatische Schmelzoberfläche entfernt wird, welche eine geringere Retention bietet. Durch das Beschleifen des Zahns verbleibt ein so genannter organischer „smear layer“ bestehend aus Hydroxylapatit, Zellpartikeln und Zellflüssigkeit auf der Zahnoberfläche. Dieser wird mittels einer 3040\%igen Orthophosphorsäure entfernt. So wird die glatte Schmelzoberfläche in ein raues, durch die Schmelzprismen gebildetes retentives Ätzmuster verwandelt. Diese Mikroretentionen verzahnen sich mit dem Bonding. Die nach der Polymerisation des Bondings entstehenden Tags in den Mikroporositäten verstärken diesen Effekt der Adhäsion (LOPES et al. 2002).

Präparation mit Dentineröffnung:

Bei Extension der Präparation bis in das Dentin muss eine Dentinkonditionierung erfolgen. Der beim Schleifen entstehende „smear layer“ verstopft die Dentintubuli, sodass die Tubuliflüssigkeit nicht entweichen kann. Dieser verhindert eine mikroretentive und chemische Haftung zwischen Dentin und Bonding und muss daher entfernt werden, um eine Adhäsion zu bewirken. Dies geschieht durch die drei Schritte des Ätzens (bzw. selbstkonditionierender Primer), des Auftragens von Adhäsiv und letztendlich des Bondings.

Das Ätzen entfernt den Smear layer und demineralisiert das Dentin, wodurch die Tubuli geöffnet werden (GWINNETT 1993). Der Primer beeinflusst/penetriert die Kollagenfasern und erlaubt so das Einfließen des Bondings in die Tubuli. Auch das Bonding hat Einfluss auf das Kollagengeflecht und bildet eine Mischzone aus demineralisiertem Dentin und ungefülltem Komposit (GORDAN et al. 1998). Diese Zone wird "Interdiffusionszone" genannt und ist ein wichtiger Faktor für die Haftkraft. Durch Zusammenfügen einiger dieser einzelnen Substanzen zu einer einzigen (self-etch-Technik) wird die Anzahl an Schritten verringert (WATANABE et al. 1994). Das selbstkonditionierende Primer-Adhäsiv-System kombiniert alle drei Schritte in einem (one-bottle-System) (YOSHIYAMA et al. 1998). 


\subsubsection{Befestigungsmöglichkeiten}

Für die Zementierung sind dualhärtende Materialien das Mittel der Wahl. Die lichthärtende Komponente erlaubt eine etwas längere Verarbeitungszeit und Applikationsdauer des Veneers als bei chemisch härtenden Zementen. Nachteil der dualhärtenden Substanzen ist die Farbunbeständigkeit laut PEUMANN et al (2000), die bei lichthärtenden besser ist. Die Lichtdurchlässigkeit der Facette sollte immer gegeben sein, um der lichthärtenden Komponente die Möglichkeit zur Polymerisation zu geben und somit eine ausreichende Härte zu erreichen (LINDEN et al. 1991). Dies ist zum Beispiel bei sehr dicken oder sehr opaken Veneers aufgrund von starken Zahnverfärbungen nicht der Fall. Hierfür ist wieder der dualhärtende Zement das Mittel der Wahl.

\subsubsection{Adhäsionskomplex Zahn-Zement-Keramik}

Wird die Keramikoberfläche konditioniert, so erhält man den stärksten Verbundkomplex (STACEY 1993). Verzichtet man auf die Kombination der drei Komponenten des Komplexes, so halbiert sich die Haftkraft: Zahn-Zement-Keramik-Komplex (63 MPa), separater Zement-Schmelz-Komplex (31 MPa), separater ZementKeramik-Komplex (33 MPa). ANDREASEN et al. (1991) sowie STOKES und HOOD (1993) stellten fest, dass extrahierte Frontzähne nach einer VeneerVersorgung die Ursprungsstabilität erreichten. MAGNE und DOUGLAS (1999C) zeigen, dass Veneers das mechanische Verhalten und die Mikrostruktur von in-vitroZähnen aufweisen, wenn sie an einer konditionierten Dentinoberfläche befestigt sind.

\subsubsection{Versorgungsarten}

Um die dentalen Kriterien der oben aufgeführten Bewertungsliste realisieren zu können, gibt es je nach Indikation diverse Möglichkeiten. Da in dieser Arbeit die Ästhetik im Bezug auf die Frontzähne betrachtet wird, soll ausschließlich das Veneer genauer beschrieben werden. Darüber hinaus stehen Versorgungen wie Kronen, Inlays, Teilkronen, die kieferorthopädische Behandlung und das Bleichen zur Verfügung. 


\subsubsection{Veneer}

Mit so genannten Veneers können leichte Zahnfehlstellungen, Zahnlücken, unbefriedigende Zahnfarben, lokale Verfärbungen sowie Schmelzhypoplasien einfach und zahnhartsubstanzschonend versorgt bzw. korrigiert werden (BOYER und CHARKLEY 1982, SCHMALZ et al. 2007). Ein Veneer ist eine hauchdünne, lichtdurchlässige Keramikschale für Zähne (überwiegend Frontzähne), die adhäsiv auf die Zahnoberfläche aufgebracht wird. Mittels Veneers erhalten die sichtbaren Oberflächen der Zähne durch Zahnfarb- und Zahnformkorrektur ein optimiertes Aussehen. Der Einsatz von Keramik-Veneers stellt generell keine gesundheitliche Gefährdung dar, weil das Keramikmaterial eine sehr hohe Bioverträglichkeit besitzt.

Die verschiedenen konservierenden/prothetischen Versorgungsmöglichkeiten setzen einen Verbund der Restaurationsmaterialien mit der Zahnhartsubstanz voraus. Bei den heutigen Verfahren werden zum einen mikromechanische Verankerungen (Retention) und zum anderen Bindungen über chemische oder physikalisch-chemische Kräfte (Adhäsion) angestrebt. Bei der Eingliederung neuzeitlicher Füllungsmaterialien, wie Komposit- und/oder Keramikrestaurationen, erfolgt eine adhäsive Befestigung an der Zahnoberfläche mittels so genannter Säureätztechnik und Verwendung von Dentinhaftvermittlern (BUONOCORE 1955, MASUHARA 1969). Keramische Restaurationen können dabei sowohl über mikromechanische Retentionen als auch über chemische Bindungen verankert werden (SIMONSEN und CALAMIA 1983). Bei auspolymerisierten Kunststoffschalen und Keramikveneers ist die Retentionskraft jedoch stark reduziert. Sie beträgt nur etwa zehn Prozent der Belastbarkeit eines plastisch aufgetragenen Materials. Die Retention muss deshalb auf dem Wege der mikromechanischen Verankerung durch Aufrauung der Keramikoberflächen und Zwischenlagerung eines haftvermittelnden niedrigviskösen Komposits, der einen adhäsiven Verbund zur Zahnoberfläche besitzt, geschaffen werden (CALAMIA 1989, CVAR und RYGE 1971, SIMONSEN und CALAMIA 1983).

Vorliegende klinische Langzeitstudien belegen, dass innerhalb von sechs Jahren nur 2\% der Keramik-Veneers verloren gehen (SCHMALZ et al. 2007, LAMPERT 1976). Wenn die Restaurationen mit zunehmender Liegezeit Gebrauchsspuren aufzeigen, so sind diese Veränderungen nach fünfjähriger Liegezeit noch dezent und eher klinisch unbedeutend (LAMPERT 1976). Aus diesem Grund gelten Veneerrestaurationen 
heute bei gezielter Indikationsstellung als geeignete Versorgungsmöglichkeiten für Ober- und Unterkieferfrontzähne.

\subsection{Geschichte der Veneer-Technik}

Der erste, der Veneers als Behandlung von Frontzähnen beschrieben hat, war CHARLES PINCUS im Jahre 1938. Er verhalf mit seiner neu entwickelten Therapie den Schauspielern in Amerika zum bis heute noch typischen „Hollywood-Smile“. Befestigt wurden die Facetten für den Auftritt vor der Kamera für einige Stunden mit einem Haftpulver.

Das Verlangen nach beständigem Halt führte durch BUONOCORE (1955) zur Entwicklung der Säureätztechnik und zusammen mit BOWEN et al. (1978) zur Verwendung von Dentinhaftvermittlern. Dabei kommt es zum Haftverbund zwischen dem konditionierten Zahn und der Kunststoffoberfläche, was die Herstellung von geschichteten Veneers aus Kunststoff möglich machte. Aufgrund der kurzen Aushärtungszeit war es für den Zahnarzt schwierig, die ästhetischen Ansprüche zu erfüllen, bevor es zur chemischen Aushärtung kam. Die später entwickelten lichthärtenden Komposite verbesserten dies jedoch, was es dem Behandelnden leichter machte, den Patientenvorstellungen gerecht zu werden.

1975 war ROCHETTE der erste, der Keramikrestaurationen im Frontzahngebiet einsetzte. Er ließ das einzusetzende Teil im Labor herstellen und behandelte so frakturierte Zähne ohne operativen Eingriff.

Im Jahre 1976 wurde von FAUNCE und MYERS (1976) das indirekte vorfabrizierte Veneer als Alternative zu den aufgeschichteten Kunststoffveneers beschrieben. Sie haben die Keramik mit einem Primer vorbehandelt, einen Kunststoffkleber aufgetragen und somit einen Klebeverbund zwischen dem Veneer und der abgeätzten Zahnoberfläche geschaffen.

SIMONSEN und CALAMIA führten 1983 die Ätzung der Keramik und die Oberflächenkonditionierung durch Silane ein und sorgten somit für einen entscheidenden Faktor zur Verbesserung des Verbundes zwischen Zahn und Veneer (CALAMIA und SIMONSEN 1984).

Bis heute hat sich an diesem Grundprinzip nichts geändert und die lediglich verbesserten Methoden sowohl der Zahnmedizin als auch der Zahntechnik werden noch heute durchgeführt. Dies ist auch TOUATI et al. (1985), TOUATI und BERSAY 
(1987), TOUATI und PLISSART-VANACKERE (1990) zu verdanken, die dieses Prinzip in Europa verbreiteten.

Als günstig ist die Kombination von Komposit mit Keramik zu bewerten, da man so die Vorteile der beiden Materialien kombiniert, die Eigenschaften somit verbessert und die Nachteile minimiert. Dies wird durch eine größere Masse an Keramik im Verhältnis zum Komposit sichergestellt (MAGNE und BELSER 2004).

In Deutschland lässt sich ein stetiger Anstieg der Verwendung von Veneers vermerken, der jedoch noch nicht so groß ist wie in den Vereinigten Staaten von Amerika (PAPE und KERSCHBAUM 1992).

\subsection{Indikationen und Kontraindikationen}

\subsection{Indikationen}

Die drei Hauptindikationen sind nach BELSER et al. (1997) die Zahnverfärbungen (Typ I), morphologische Defizite (Тур II) und umfangreiche Schädigungen (Тур III).

Die in der Tabelle 2 aufgeführten Indikationen sind nach und nach hinzugekommen. So kann man sagen, dass Typ I und zum Teil Typ II schon lange mit Keramikrestaurationen versorgt wurden (HORN 1983A, CALAMIA 1983, CALAMIA 1985, CALAMIA 1989, CALAMIA 1993), der weitere Teil des Typs II und Typ III hingegen erst seit kurzem (ANDREASEN et al. 1991, ANDREASEN et al. 1992, BELSER et al. 1993, BELSER et al. 1997, MAGNE und DOUGLAS 1999A, MAGNE und DOUGLAS 1999B, MAGNE und DOUGLAS 1999C, MAGNE und HOLZ 1994, MAGNE et al. 2000, WALLS 1995A, WALLS 1995B). 
Tab.2: Indikationen für Keramikverblendungen

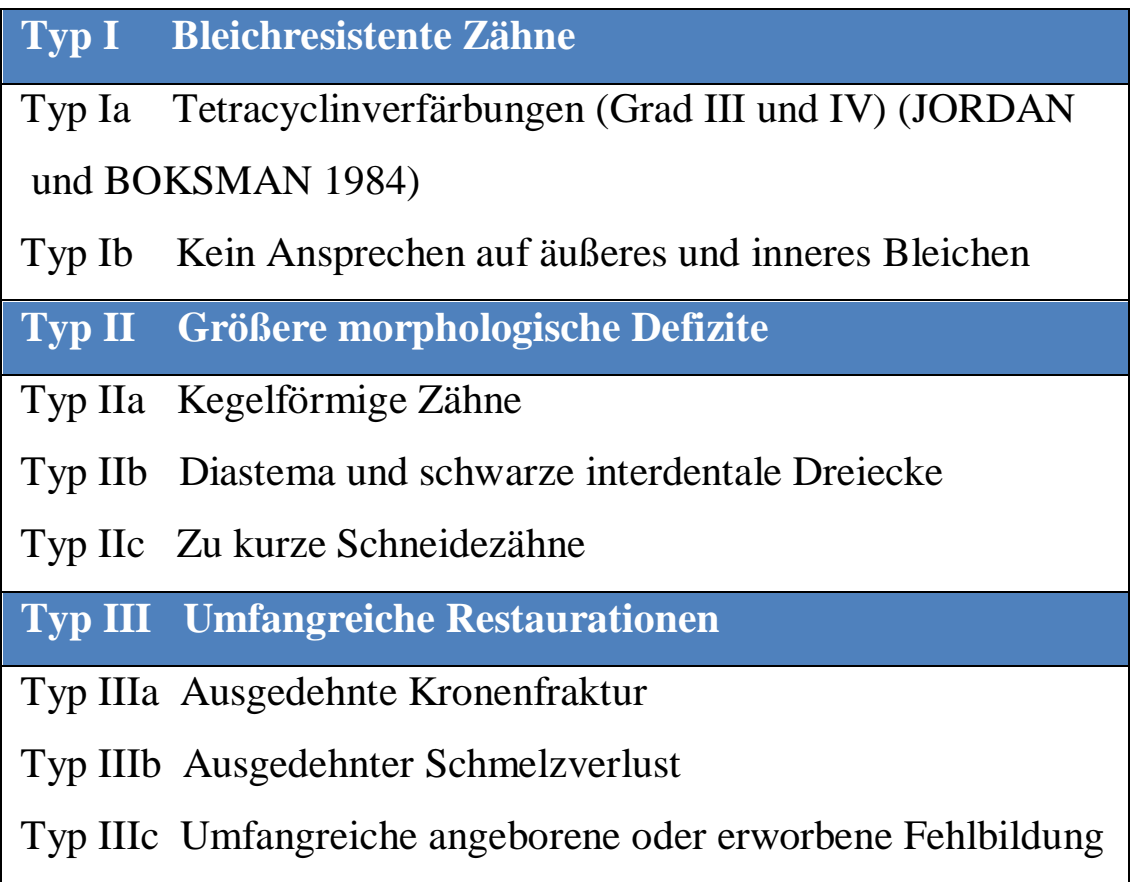

Klinisch haben sich nach JÄGER und WIRZ (1992) für die Laminierungen folgende Indikationsgebiete herausgestellt:

- $\quad$ Form- bzw. Stellungsverbesserungen im Frontzahngebiet, nicht so langwierig wie eine kieferorthopädische Behandlung

- Abrasionen/exzentrische Schlifffacetten auf den Inzisalkanten

- $\quad$ Frakturen des Schmelzes/Dentins ohne Pulpabeteiligung

- $\quad$ Reparaturen an Verblendbrücken.

HORN (1983B) fügt noch die Anfertigung und Befestigung individuell hergestellter Brackets für die Kieferorthopädie sowie die Formänderung von Pfeilerzähnen für die Aufnahme von Halteelementen für herausnehmbare Modellgussprothesen hinzu.

Die Veränderung der sagittalen Frontzahnführung für gnathologisch prothetische Rekonstruktionen wird als weiterer Punkt von LEVIN (1988) ergänzt.

Als weitere Indikation ist trotz des limitierenden Faktors der großen Kräfte, der größeren funktionellen Belastung im Vergleich zum Oberkiefer und der okklusalen Interferenzen von MOURADIAN et al. (1976) sowie MOURADIAN et al. (1978) der Einsatz von Veneers in der Unterkieferfront beschrieben worden. 
CALAMIA (1988) erwähnt ebenso die Amelogenesis imperfecta und die intrinsischen Verfärbungen und BERTOLOTTI (1989) die Neu-Rekonstruierung, das Verlängern und das Umformen des Zahnes.

Oft gibt es nicht nur eine Indikation, sondern bei genauer Betrachtung der Zähne mehrere. So kann z. B. bei einem Gebiss mit einem devitalen Zahn, einem Diastema und abradierten Zähnen mit der Facettentechnik ein befriedigendes Ergebnis für all diese Faktoren erzielt werden (MAGNE und BELSER 2004).

Wichtig für die Planung der Therapie ist ebenfalls der biologische Aspekt. Man muss sich immer vor Augen halten, dass für die Behandlung mit einer Vollkrone wesentlich mehr Zahnhartsubstanz, die auch noch gesund ist, geopfert werden muss. Dies hat ebenfalls Auswirkungen auf die Pulpa, das Zahnfleisch und die Biomechanik der Krone (MAGNE und BELSER 2004).

CALAMIA (1989) hat die Verträglichkeit von adhäsiv befestigten Keramikrestaurationen im Hinblick auf die parodontale Verträglichkeit positiv bewertet. Auch KOURKOUTA et al. (1994) zeigten den positiven Effekt im Bezug auf den Plaqueindex.

\subsection{Kontraindikationen}

An Kontraindikationen werden von PAPE und KERSCHBAUM (1992) und JÄGER und WIRZ (1992) folgende aufgelistet:

- $\quad$ Bruxismus (SHEETS und TANIGUCHI 1990, FRADEANI und BARDUCCI 1996)

- $\quad$ Kopfbiss (SHEETS und TANIGUCHI 1990, FRADEANI und

BARDUCCI 1996)

- $\quad$ Große keilförmige Defekte

- Geringe Restzahnhartsubstanz, viele Füllungen

- $\quad$ Habits (Knirschen, Pressen, Bleistiftkauen).

CASTELNUOVO et al. (2000) beschreiben als limitierende Faktoren einen großen Zahnhartsubstanzverlust, großflächige Karies, große bzw. viele Füllungen. Diese Schäden sollten eher mit einer Vollkrone behandelt werden, da die benötigte Retentionsfläche für ein Veneer bzw. die Konditionierungsfläche nicht ausreicht, um eine Langlebigkeit zu gewährleisten. Des Weiteren werden eine schlechte Mundhygiene, 
die zu Parodontopathien führt, sowie eine schlechte Compliance als Faktoren aufgelistet, die zur Ablehnung der Behandlung mit Veneers führen sollten.

\subsection{Vorteile und Nachteile}

\subsection{Vorteile}

Gegenüber den direkten und indirekten Kompositverfahren weisen Keramikveneers eine erheblich bessere Ästhetik und Haltbarkeit auf, sie sind dauerhafter als direkte Kompositrestaurationen im Hinblick auf die Ästhetik (NIXON 1990).

Nicht zu vernachlässigen ist das Kriterium der Substanzschonung, das bei der Präparation des Zahnes für ein Veneer ein großer Vorteil im Vergleich zu einer Vollkrone ist (SHEETS und TANIGUCHI 1990, PERMANN et al. 1989, GARBER 1989, NIXON 1990, PAPE und KERSCHBAUM 1992, JÄGER und WIRZ 1992, GOLDSTEIN 1990).

Auch ausgedehnte Korrekturen sind mit Hilfe von Keramikfacetten leichter möglich (McLAUGHLIN 1988).

Form und Farbe können durch die Eingliederung von Porzellanfacetten verändert bzw. verbessert werden (BOYER und CHALKLEY 1982). Die Keramikfacetten haben gegenüber der Kompositlaminierung folgende Vorteile (SHEETS und TANIGUCHI 1990, PERMANN et al. 1989, GARBER 1989, NIXON 1990, PAPE und KERSCHBAUM 1992, JÄGER und WIRZ 1992, GOLDSTEIN 1990):

- $\quad$ Hervorragende Ästhetik

- $\quad$ bessere Farbgebung,

- $\quad$ größere Stabilität in der Mundhöhle,

- $\quad$ uneingeschränkte Biokompatibilität,

- $\quad$ große Abrasionsfestigkeit

- $\quad$ keine Flüssigkeitsaufnahme

- Verlagerung von komplizierten Arbeitsgängen vom Behandlungsstuhl in ein Labor, wo schwierige Modellierarbeiten ohne Zeitdruck vorgenommen werden können

- Vitalität bleibt erhalten. 
HORN (1983B) ergänzt die gute Biokompatibilität, die guten Haftwerte durch mikromechanische Oberflächen nach dem Ätzen sowie die Resistenz gegen alkoholische Lösungen, Medikamente, Farbstoffe sowie Kosmetika.

Keramiken bieten sich an, da sie über eine hervorragende Ästhetik, eine zahnschmelzähnliche Oberfläche, gute Randstabilität, geringe Zahnfleischreizung verfügen (GOLDSTEIN 1990).

Geringe Plaqueaffinität ist ebenfalls als Vorteil zu erwähnen (CHAN und WEBER 1986).

\subsection{Nachteile}

LAMPERT und VAN GOGSWAARDT (1995) listen als Nachteile das Ausbleiben von Reparaturmöglichkeiten, die Bruchgefahr, die hohen Kosten und die Notwendigkeit von mehreren Sitzungen auf.

Der Nachteil der Sprödigkeit ist wichtig hinsichtlich der Planung, da Spannungen, die den Werkstoff belasten, nicht durch Verformungen kompensiert werden (VAN DER ZEL 1988).

Nicht unbedingt als Nachteile, jedoch als Schwierigkeiten beim Behandlungsablauf, die zu einer größeren Anzahl Sitzungen führen, sind laut VAN THOOR (1998) folgende Punkte zu erwähnen:

- $\quad$ Ungenügende Befestigung

- Inadäquate Befestigungsmaterialien

- Zeitaufwendiges und schwieriges Behandlungskonzept

- $\quad$ Unrealistische Langzeiterfolgserwartungen der Patienten (SHEETS und TANIGUCHI 1990, PERMANN et al. 1989, GARBER 1989, GARBER et al. 1989, VAN GOGSWAARDT 1989, NIXON 1990, JÄGER und WIRZ 1992)

- $\quad$ Ergebnis vom zahntechnischen Labor abhängig

- $\quad$ Farbe des Veneers ist kaum zu verändern

- $\quad$ Aufwendige Befestigung

- Hohe zahntechnische und zahnärztliche Kosten. 


\subsection{Präparationsformen}

Um eine Langlebigkeit des Veneers zu ermöglichen, ist als einer der wichtigsten Faktoren die exakte Präparation zu nennen.

Die Ziele dabei sind:

- $\quad$ ausreichender Substanzabtrag, um eine Überkonturierung durch den Zahntechniker zu vermeiden

- $\quad$ gleichzeitig so viel Zahnhartsubstanz erhalten und schonen, wie möglich

- $\quad$ Kanten abrunden, da diese Spannungen bewirken, die später zu Frakturen führen können

- $\quad$ die Präparation so weit extendieren, dass die Schmelz-Keramik-Grenze im nicht sichtbaren Bereich liegt

- $\quad$ spannungsfreie Insertion ermöglichen.

Aus diesen Punkten ergeben sich vier wesentliche Aspekte, die die optimale Präparation beschreiben und systematisch abgearbeitet werden sollten.

\section{Zahnhartsubstanzabtrag/Tiefe der Präparation}

In den letzten Jahren hat sich die Meinung zum Thema Substanzabtrag stark geändert. War man früher noch der Meinung, den Schmelz nur geringfügig abzutragen, also nur anzurauen (CALAMIA 1983, HORN 1983A, GARBER et al. 1989), so ist man heute der Auffassung, dass ein Minimum an Präparation notwendig ist. Ein Grund ist der, dass dem Zahntechniker ein gewisser Spielraum für die ästhetische Gestaltung gegeben werden sollte. Hierdurch wird eine Überkonturierung vermieden und so das Parodontium geschont (WALLS et al. 2002). Des Weiteren geht man davon aus, dass die fehlende Präparation, zur Schonung der Zahnhartsubstanz, zu einer höheren Frakturrate führt, als bei einem vorherigen Abtrag (SHAINI et al. 1997). Andere Kriterien, die für eine Präparation sprechen, sind die erleichterte Insertion beim Zementieren durch genaue Vorgabe der Position (SCHNEIDER et al. 1981, GILMOUR und STONE 1993), ausreichend Platz für den Opaker bei stark verfärbten Zähnen sowie für die adhäsiven Befestigungsmaterialien, die für die Haftkraft zwischen Zahn und Keramik verantwortlich sind. Für Letzteres ist es wichtig, die oberflächliche aprimatische Schmelzschicht zu entfernen, die nicht im gewünschten Ausmaß auf die Ätzung anspricht (SCHNEIDER et al. 1981). 
Die Grenze der Präparation sollte sich jedoch immer im Bereich des Schmelzes befinden. Von TROEDSON und DERAND (1998), HAHN et al. (2000), PEUMANS et al. (2000) wird eine Reduktion des Schmelzes um 0,5 mm beschrieben. Dies ist einerseits ausreichend für das Adhäsiv und andererseits schonend genug für den Schmelz. Außerdem wird so eine durch den Zahntechniker dünn ausgearbeitete Keramikfacette ermöglicht, wodurch die Transluzenz und somit die Ästhetik erhöht wird. Laut CHRISTENSEN GJ und CHRISTENSEN RP (1991) liegt das Optimum beim Abtrag von 0,75 mm. Im Gegensatz dazu beschreiben FERRARI et al. (1992), dass auf Gingivaniveau ein Abtrag von maximal 0,5 mm bei Frontzähnen ohne Erreichen des Dentins aufgrund der geringen Schmelzdicke in diesem Bereich nicht möglich ist. Ein Substanzabtrag von 0,5 mm oder weniger ist NATTRESS et al. (1995) zu Folge bei einer freihändigen Präparation im approximal-zervikalen Bereich in der Mehrzahl der Fälle nicht möglich. Dies bedeutet eine ungewollte Eröffnung des Dentins.

\section{Abtrag der Inzisalkante}

WALLS et al. (2002) beschreiben vier Möglichkeiten der inzisalen Beschleifung des mit einem Veneer zu versorgenden Zahns:

- $\quad$ Gefenstert (Window), wobei die Inzisalkante des natürlichen Zahns erhalten bleibt. Vorteil dieser Präparation ist das Belassen der Schmelzschicht in diesem Bereich. Hierbei ist es jedoch schwierig, die Schmelz-Keramik-Grenze im unsichtbaren Bereich zu gestalten.

- $\quad$ Gefiedert (Feather), wobei das Veneer das Niveau der Inzisalkante erreicht, jedoch diese nicht reduziert wird. So behält der Zahn zwar die anteriore Führung, ist jedoch im Bezug auf Scher-/Reibekräfte bei der Protrusion sehr labil.

- $\quad$ Angeschrägt (Bevel), wobei in bukko-palatinaler Richtung die Inzisalkante angeschrägt wird. So ist eine bessere ästhetische Anpassung möglich und die korrekte Eingliederung beim Zementieren erleichtert. Des Weiteren ist der beim Feather-Veneer erwähnte Risikofaktor der Abplatzungen durch Scherkräfte reduziert. Nachteil ist der stärkere Substanzabtrag im Gegensatz zu den ersten beiden Techniken.

- $\quad$ Überlappend (Overlap), wobei die Inzisalkante am stärksten reduziert und die Präparation bis in den palatinalen Bereich extendiert wird. Die Positionierung 
während der Befestigung ist hier am deutlichsten vorgegeben. Das Veneer muss von bukko-inzisal eingesetzt und nicht wie die anderen nur von bukkal aufgeklebt werden.

HIGHTON et al. (1987) beschreiben, dass die inzisale Schmelzreduktion den Stress, dem das Veneer ausgesetzt ist, minimiert und somit die Haltbarkeit/Langlebigkeit verbessert.

Dagegen sprechen die von verschiedenen Autoren beschriebenen Stabilitätswerte der unterschiedlichen Präparationen. Laut HUI et al. (1991) liegen diese für die WindowVeneers bei 1190 N, für die Feather-Veneers bei 788 N und das Overlap-Veneer bei $688 \mathrm{~N}$.

In der Studie von CASTELNUOVO et al. (2000) frakturiert ein unrestaurierter Oberkieferfrontzahn bei 310 N, das Bevel-Veneer bei 274 N, das Feather-Veneer bei $237 \mathrm{~N}$, das Vollveneer bei $192 \mathrm{~N}$ und das Overlap-Veneer bei $164 \mathrm{~N}$.

HAHN et al. (2000) geben Werte von 653 N für unbehandelte Unterkieferfrontzähne, $693 \mathrm{~N}$ für die Feather-Veneers und $466 \mathrm{~N}$ für die Overlap-Veneers an.

WALL et al. (1992) und MEIJERING et al. (1997), sehen keinen nennenswerten Unterschied bei den vier Techniken im Bezug auf die Frakturrate.

\section{Approximale Extension}

Die Approximalkontakte sollten immer erhalten bleiben. Ist die Indikation für ein Veneer das Verschließen eines Diastemas oder eine Korrektur der Zahnform, so sollte die Interproximale Kontaktfläche präpariert werden (BELSER et al 1997). Hierdurch ist es für den Zahntechniker leichter, das allgemeine Zahnprofil des Patienten zu übernehmen. Ebenfalls das Bonden und Zementieren ist durch die von allen Seiten zu erreichenden Restaurationsränder erleichtert (BELSER et al. 1997, MAGNE und BELSER 2002). Die Stabilität im Bereich der Approximalräume ist bis heute nicht untersucht worden. 


\section{Präparationsgrenze}

Die Präparationsgrenze sollte eine optimale Eingliederung der Restauration ermöglichen.

Laut TROEDSON und DERAND (1999) ist eine Anschrägung im approximalen und zervikalen Bereich kontraindiziert. Es sollte eine Stufe bzw. Hohlkehle ohne scharfe Kanten angestrebt werden.

MAGNE und DOUGLAS (1999C) erwähnen eine kleine palatinale Stufe, die vorteilhaft ist, um die Belastung auf dünn auslaufende Ränder zu minimieren.

\subsection{Klassifizierung der Keramik-Systeme für Veneers}

Im Hinblick auf die Herstellung der Keramik-Veneers können die im Folgenden beschriebenen Systeme verwendet werden.

Bei der Prozedur der gesinterten Keramik (z. B. Feldspat-Keramik und Optec ${ }^{\circledR}$ ) wird einem Keramikpulver Wasser beigemengt, um eine wässrige Masse zu bekommen. Die Keramik wird auf einer Platinfolie oder einer feuerfesten Form aufgeschichtet. Das Pulver ist in verschiedenen Farbtönen und Transluzenzen sowie beigemengten charakteristischen Flecken und Glasuren erhältlich.

Die infiltrierten Keramiken (z. B. In-Ceram Spinel ${ }^{\circledR}$ ) bestehen aus zehn Komponenten. Zum einen aus dem Pulver, das porös ist und somit eine Infiltration mit Glas, die zweite Komponente, bei hohen Temperaturen zulässt. Im Anschluss wird das Produkt noch mit einer Feldspatkeramik verblendet.

Bei der Technik der gegossenen Keramik (z. B. Dicor ${ }^{\circledR}$ und Cera-Pearl ${ }^{\circledR}$ ) werden Keramikblöcke, die zur Herstellung von schon konturierten Restaurationen dienen, verwendet. Hierbei wird das „lost-wax-Verfahren“ und die Zentrifugalkraft ausgenutzt.

Beim Verfahren zur Herstellung gepresster Keramik (z. B. IPS Empress ${ }^{\circledR}$ ) werden die Keramikblöcke bei hohen Temperaturen geschmolzen und dann in eine Gussform gepresst, wobei wiederum das „lost-wax-Verfahren“ zum Tragen kommt.

Aus Rationalisierungsgründen hat man mittlerweile Maschinen entwickelt, um aus einem Keramikblock die Restauration computergesteuert (CAD/CAM-Systeme, Computer Aided Design / Computer Aided Manufakturing, wie z.B. Cerec ${ }^{\circledR}$, Proce- 
$\mathrm{ra}^{\circledR}$-AllCeram-System) oder mechanisch mit Hilfe von sog. Kopierschleifern (z.B. Celay ${ }^{\circledR}$-System) herauszufräsen bzw. herauszuschleifen. Somit ist es möglich, den Patienten in einer Sitzung zu behandeln.

Das Kopierschleifen gehört zu den sog. Machinable Ceramic Systems (MCS). Nicht über einen optischen Abdruck sondern über einen manuell durchgeführten Abtastvorgang wird die Information erfasst. Dies unterscheidet dieses Verfahren von den im Anschluss beschriebenen computergestützten Systemen. Ein großer Vorteil besteht hierbei darin, dass keine Abformung und Modellherstellung notwendig sind und somit mögliche Fehlerquellen entfallen.

Aus zwei wesentlichen Gründen ist das direkte Sintern auf Stümpfen und die Verwendung von den oben genannten Press- und Gusstechniken nicht möglich. Zum einen ist die Verarbeitungstemperatur von Zirkoniumoxidkeramiken zu hoch und zum anderen ist Zirkoniumoxid sehr hart, wodurch die „einfachere Verarbeitungsform“ verhindert wird. Für diese Fälle ist die CAD/CAM-Technologie das Mittel der Wahl (LUTHARDT et al. 1998).

Die Herstellung von vollkeramischen Restaurationen durch CAD / CAM Systeme erfolgt in drei Arbeitsstufen:

1. Scannen: Mit einem mechanischen oder optischen Scanner werden die digitalen Daten der zu rekonstruierenden Region gesammelt („Punktewolke“).

2. CAD: Auf dem in digitaler Form vorliegenden Modell wird mittels einer Software ein dreidimensionaler virtueller Zahnersatz entwickelt.

3. CAM: Mit einer CAM-Maschine wird aus einem industriell hergestellten Keramikblock das entstandene virtuelle Gerüst heraus gefräst.

Die große Zeitersparnis kommt dadurch zustande, dass auf die Abformung der präparierten Zähne, das Herstellen eines Meistermodells sowie das Aufwachsen, Einbetten und Gießen der Restauration verzichtet werden kann, weil die Abtastung der Kavität im Mund des Patienten erfolgt. Danach wird die vollkeramische Restauration aus dem vorfabrizierten Keramikblock direkt heraus geschliffen. 


\subsection{Erfolgskriterien und -aussichten}

\subsection{Anforderungen}

Der Erfolg der Restauration mittels Veneers ist laut „Counsil on Dental Materials and Devices“ von 1984 abhängig von den folgenden Kriterien:

1. Die Zahnform sollte erhalten bleiben, um keine gingivalen Reizungen hervorzurufen.

2. Die Präparationsgrenze sollte bis zur Gingiva oder etwas subgingival gelegt werden.

3. Alle Ränder des Veneers sollten glatt und hochglanzpoliert sein.

4. Die Kompositbefestigungsfuge sollte polierbar sein.

5. Das Veneer sollte Verfärbungen von Zähnen maskieren können, ohne die Restauration überzukonturieren.

6. Die Veneer-Technik sollte es ermöglichen, die Basisfarbe und die inzisale Transparenz zu variieren, um hohen ästhetischen Ansprüchen gerecht zu werden.

7. Durch das Befestigen und Polieren sollten keine Schäden an den angrenzenden Zahnstrukturen entstehen.

8. Die Artikulation und Okklusion sollte so möglich sein, dass keine großen Kräfte auf das Veneer übertragen werden.

9. Das Kunststoffveneer sollte im normalen Funktionsablauf nicht frakturieren (bzw. es sollte einfach zu reparieren sein).

10. Das Kunststoffveneer sollte harmonisch und natürlich aussehen.

11. Das Kunststoffveneer sollte resistent sein gegen Zahnputzmittel und leicht zu reinigen.

12. Die Zahnhartsubstanz sollte so wenig wie möglich reduziert werden.

13. Es muss Zahnschmelz vorhanden sein, um einen guten Verbund zum Komposit-Befestigungsmaterial über die Säure-Ätz-Technik zu erzielen.

14. Freiliegendes Dentin sollte geschützt werden, um postoperative Schmerzen und Nervenreizungen zu vermeiden. 
Speziell für Keramikveneers stellten JÄGER und WIRZ (1992) ergänzende Kriterien auf:

1. Kritische Indikationsstellung, Erkennen von Kontraindikationen

2. Beurteilung des Schmelzangebots

3. Präparationstechnik und Wahl der Präparationsgrenze

4. Abformung, Modellherstellung, Zahntechnik

5. Konditionierung der Werkstücke

6. Optimales Arbeitsfeld mit Konditionierung der Schmelzpartie

7. Passgenauigkeit, Verbundfestigkeit, Befestigung und Ausarbeitung

8. Zeitaufwand, Kostenvergleiche

Der Grund für die verschiedenen Mineralisationsverhältnisse und somit zwei unterschiedlich harte Substanzen Schmelz und Dentin ist der Widerstand, den sie gegenüber Kau- und thermischen Belastungen aufbringen müssen. Die beiden Strukturen, der sehr harte Schmelz und das etwas „weichere“ Dentin, stehen in einer strukturellen und physikalischen Beziehung.

Schon bei der kleinsten strukturellen Veränderung, also auch das Beschleifen für ein Veneer, wird dieser Zustand negativ beeinflusst und muss einkalkuliert werden.

MAGNE und DOUGLAS (1999C) demonstrierten, dass die Biegefestigkeit wiederhergestellt wurde, wenn die beschliffenen Zähne mit FeldspatkeramikRestaurationen bzw. Veneers versorgt wurden. Im Frakturtest wiesen die mit Veneers versorgten Zähne eine erhöhte Steife auf (MAGNE und DOUGLAS 1999D). Dies bedeutet jedoch nicht, dass ein Veneer die Stabilität eines Zahnes aufweist. Die Anfälligkeit von Veneers gegenüber Frakturen hängt von vielen Faktoren ab. Die Stabilität der Facette nimmt mit der Anzahl und dem Grad an inneren Bruchspalten ab, die vor, während und nach dem Zementieren auftreten können. Diese liegen in fast allen Fällen unabdingbar vor. Verursacht werden sie durch Kondensation, Schmelzen und dem Sinterungsprozess aufgrund von unterschiedlichen Wärmeausdehnungsgraden im Kern und am Rand der Restauration. Aber auch das Beschleifen, Ausarbeiten und Polieren des Veneers sorgt für innere Spannungen (HONDRUM 1992). Als weitere Ursachen beschreiben MAGNE et al. (1999) das Einsetzen unter Spannung, die Polymerisationsschrumpfung und das Thermocycling. Beim Einsetzen kann es zum Verkanten und zu Zugkräften kommen, die wiederum Spannungen und kleine Defekte verursachen (HONDRUM 1992). Diese Mikrorisse wachsen langsam 
bei bestehendem Stress bis zu einem kritischen Punkt, in dem es zum schnellen Entladen der Spannung und so zu einem schnellen Frakturprozess kommt (RITTER 1995).

Auch das Aushärten beim Klebeverbund sorgt für Zugspannungen auf die Keramikoberfläche durch den Schrumpfungsprozess des Bondings und des Zements. Somit ist das Einprobieren und Zementieren ein sehr sensibler Schritt in der ganzen Veneerprozedur. Das Verhältnis der einzelnen Schichten beim Bonden und beim Zementieren scheint einen erheblichen Einfluss auf die Spannungsverteilung im Keramikveneer zu haben. Liegt eine sehr dünne Keramikschicht mit einer dicken Zementschicht vor, so ist die Wahrscheinlichkeit der Fraktur sehr groß (MAGNE et al. 1999).

Die thermische Belastung auf die Keramik ist nicht zu vernachlässigen. Im Mund variieren die Temperaturen zwischen $1^{\circ} \mathrm{C}$ (anteriore Maxilla) und $58,5^{\circ} \mathrm{C}$ (posteriore Mandibula) (PALMER et al. 1992). Beim Thermocycling von Dentalmaterialien werden Werte von $0^{\circ} \mathrm{C}$ bis $\mathrm{zu} 67^{\circ} \mathrm{C}$ erreicht. Aufgrund der verschiedenen Wärmeausdehnungskoeffizienten der Keramik und des Zahnes entstehen Stressmomente, die sich auch durch Risse bemerkbar machen können.

Zwischen der Keramikoberfläche, die durch die Risse freigelegt wird, und Wasserdampf kann es zu chemischen Reaktionen kommen, die wiederum zu Spannungen und somit Risswachstum führen (HONDRUM 1992).

Mit der Beißkraft eines Menschen korrelieren diverse Parameter wie Geschlecht, Alter, Bezahnung, Muskelkraft, Trainingszustand und Bruxismus. Die Beißkräfte liegen je nach Zahn zwischen 137 N (Frontzahn) und 147 N (Eckzahn). Maximal wurden bei Männern in der Fronzahnregion $176 \mathrm{~N}$ und bei Frauen $108 \mathrm{~N}$ gemessen (HELKIMO et al. 1977). Laut SONNENBURG et al. (1978) liegen die Werte der maximalen Beißkraft bei Männern zwischen 215 N und $360 \mathrm{~N}$ und bei Frauen zwischen $115 \mathrm{~N}$ und $268 \mathrm{~N}$.

\subsection{2 Überlebens-und Erfolgsrate}

Um eine bestimmte Technik oder ein neues Produkt weiterempfehlen zu können, ist es wichtig, Langzeitergebnisse zu haben. So kann objektiv beurteilt werden, welchen Erfolg man erzielt hat und wie lange dieser voraussichtlich anhält, ehe die Restauration verloren geht. Laut STRUB (1992) sind Fünf-Jahres-Ergebnisse aus der klini- 
schen Arbeit vor einer Weiterempfehlung für die Praxis nötig. PRÖBSTER (1996) beschreibt eine Restauration als erfolgreich, wenn es eine Überlebensrate von 95\% nach fünf Jahren und 85\% nach zehn Jahren aufweist.

Im Bezug auf Veneers ist die Überlebensrate abhängig von den Faktoren Fraktur, Lösen der Facette, Abplatzungen, Abschilferungen, Gingivaadaptation, Sekundärkaries, Verfärbungen und Überkonturierungen. In den vielen Studien wird von einer geringen Fehlerquote von null bis fünf Prozent berichtet, ausgelöst durch Fraktur und Haftkraftverlust (CLYDE und GILMOUR 1988, STRASSLER und NATHANSON 1989, RUCKER et al. 1990, NORDBO et al. 1994, KIHN und BARNES 1998, PEUMANS et al. 1998, DUMFAHRT und SCHAFFER 2000).

Die Verlustrate liegt auch in anderen Studien bei null bis drei Prozent mit der Fraktur als Hauptursache (MAGNE und DOUGLAS 2000, PEUMANS et al. 2004, DUMFAHRT und SCHAFFER 2000, GUESS und STAPPERT 2008, MURPHY et al. 2005, FRIEDMAN 1998, LAYTON und WALTON 2007).

CHRISTENSEN G und CHRISTENSEN R (1991) berichten von einer Misserfolgsrate von 13\% nach drei Jahren und STRASSLER und WEINER (1995) von 7\% nach sieben bis zehn Jahren.

Nach viereinhalb bis sechs Jahren Beobachtung wurde in anderen Untersuchungen eine Überlebenswahrscheinlichkeit von 96,5-98,8\% festgestellt (FRADEANI 1998, ARISTIDIS und DIMITRA 2002, GUESS und STAPPERT 2008).

Diverse in-vivo-Studien, die die Gingivaverhältnisse und das Parodontium beurteilen, beschreiben gute bis sehr gute Ergebnisse (65-98\%) (STRASSLER und NATHANSON 1989, RUCKER et al. 1990, CHRISTENSEN G und CHRISTENSEN R 1991, STRASSLER und WEINER 1995, KIHN und BARNES 1998, MEIJERING et al. 1998, DUMFAHRT und SCHAFFER 2000).

JÄGER et al. (1995) und PEUMANS et al. (1998) hingegen haben hier schlechtere Ergebnisse mit leichten Gingivaveränderungen am Veneerrand festgestellt.

GUESS und STAPPERT (2008) berichten von einer Erfolgsrate von 72\%-85\% nach fünf Jahren Beobachtungszeit. Hauptursache für den Misserfolg waren notwendige Rezementierungen. Hier spricht man von einer Rezementierungsrate von bis zu 11\% (MURPHY et al. 2005, DUNNE und MILLAR 1993, FRIEDMAN 1998). Die Rate an devitalisierten bzw. Wurzelkanal zu behandelnden Zähnen ist als gering zu bezeichnen (DUMFAHRT und SCHAFFER 2000, MAGNE und DOUGLAS 2000, GUESS und STAPPERT 2008, PIPPIN et al. 1995). 


\section{Material und Methoden}

\subsection{Administrative Vorbereitungen}

Das Studiendesign und der Antrag für das Forschungsvorhaben wurden mit dem ehemaligen Direktor der Abteilung Medizinische Statistik der Universitätsmedizin Göttingen, Prof. Dr. rer. nat. Brunner, vor Studienbeginn besprochen.

Die Erlaubnis zur Durchführung der Studie wurde bei der Ethik-Kommission der Georg-August-Universität Göttingen beantragt und durch den Vorsitzenden unter der Antragsnummer 16/1/09 genehmigt (s. Anhang).

Die Freiwilligkeit der teilnehmenden Probanden war Voraussetzung für diese Untersuchung. Die Probanden wurden angeschrieben und um Teilnahme an der Nachuntersuchung gebeten. In einem Eingangsgespräch wurden die Patienten über die Studie aufgeklärt und gebeten, die Einverständniserklärung zu unterschreiben. Durch die Einwilligungserklärung wurde die freiwillige Teilnahme der Probanden erfasst.

\subsection{Material}

\subsubsection{Probanden}

\subsubsection{Probandenverwaltung und -dokumentation}

Die personenbezogenen Daten unterlagen dem Datenschutz und wurden vom Leiter der Prüfung nicht weitergegeben. Sie wurden pseudoanonymisiert behandelt, nur zu Untersuchungszwecken verwendet und nach der Auswertung vernichtet. Nach erfolgtem Widerruf wurden die personenbezogenen Daten unverzüglich gelöscht. Zur statistischen Auswertung wurden die anonymisierten Daten in eine Exceltabelle übertragen. Auf die Daten hatten nur der Leiter der Prüfung und die Prüfärzte Zugriff; die Daten waren durch ein Passwort gesichert. Die Prüfbögen (Erfassungsbögen CRF`s) wurden in einem Prüfordner gesammelt und beim Leiter der Prüfung für 15 Jahre aufbewahrt. Zum Ausschluss von Probanden mit Risikoerkrankungen erfolgten eine Allgemeinanamnese sowie die Inspektion der Mundhöhle. Wenn nach diesen Schritten bereits eine Teilnahme an der Studie ausgeschlossen wurde, wurde die Er- 
fassungsuntersuchung nicht fortgesetzt. Die Dokumentation der erhobenen Befunde erfolgte gemäß Prüfplan mittels CRF-Prüfbögen.

Alle Patienten, deren Daten analysiert wurden, wurden durchnummeriert und die Datenblätter (CRFs) pseudoanonymisiert. Jeder Proband konnte jederzeit ohne Angabe von Gründen von der Teilnahme an der Studie zurücktreten.

\subsubsection{Probandenauswahl}

Alle Patienten die an der Studie teilnahmen, hatten folgende Voraussetzungen zu erfüllen:

- Patienten der Zahnarztpraxis Dr. med. dent. S. Rinke \& S. Schmidt

- im Zeitraum vom 01.01.1998 bis zum 31.12.2005 mit Veneers aus Presskeramik (Cergo DeguDent, Hanau/Deutschland) in der Ober- und Unterkieferfront (13-23 und 33 - 43) versorgt

- freiwillige Teilnahme

- positive Vitalität der zu versorgenden Zähne

- funktionsdiagnostisch unauffällig

- maximal über zwei Flächen verlaufende Kompositfüllung.

\subsection{Einschlusskriterien}

Es wurden nur die Zähne in die Untersuchung mit aufgenommen, die maximal zweiflächige Kompositfüllungen aufwiesen. Außerdem durfte nur eine der folgenden Indikationen Anlass für die Behandlung sein:

- Diastema

- lokale Zahnverfärbung

- unbefriedigende Zahnfarbe

- hypoplastische Zähne

- Zahnfehlstellung. 


\subsection{Ausschlusskriterien}

Ausgeschlossen wurden Probanden, bei denen einer der folgenden Punkte zutraf:

- Funktionsstörungen

- große keilförmige Defekte

- Probanden unter 18 Jahren

- immunsupprimierte Patienten

- organtransplantierte Patienten

- Patienten, bei denen eine Endokarditisprophylaxe erforderlich gewesen wäre

- Vorliegen von Hepatitis A, B, C, TBC, HIV

- Patienten mit Niereninsuffizienz

- Patienten mit Anfalls- oder Nervenleiden

- suchtkranke Patienten

- bekannte Unverträglichkeit/Überempfindlichkeit

- vorliegende Schwangerschaft.

\subsubsection{Veneers}

\subsubsection{Präparation}

Alle hier untersuchten Veneers wurden vom selben Behandler präpariert und eingesetzt.

Als Präparationsset wurde das ERGO 4395 von Komet benutzt.

Vestibulo-zervikal wurde mit einer Tiefe von 0,3-0,5 mm in Form einer Hohlkehle präpariert. Die vestibuläre Fläche wurde um 0,5-0,7 mm abgetragen. Die Inzisalkante wurde in Form einer palatinalen Overlap-Präparation um 1-1,5 mm reduziert, wobei möglichst der Kontakt zum Antagonisten bei maximaler Interkuspidation aufgelöst wurde. War dies nicht möglich, so wurde die präparierte Fläche um $1 \mathrm{~mm}$ nach palatinal extendiert. Die durch die Präparation entstandenen scharfen Kanten wurden gebrochen, sodass zwischen der labialen Fläche und der Inzisalkante ein Winkel von 110-130 entstand (s. Abb.2). Vorhandene Approximalkontakte wurden entfernt und mit der Keramik wieder aufgebaut. 


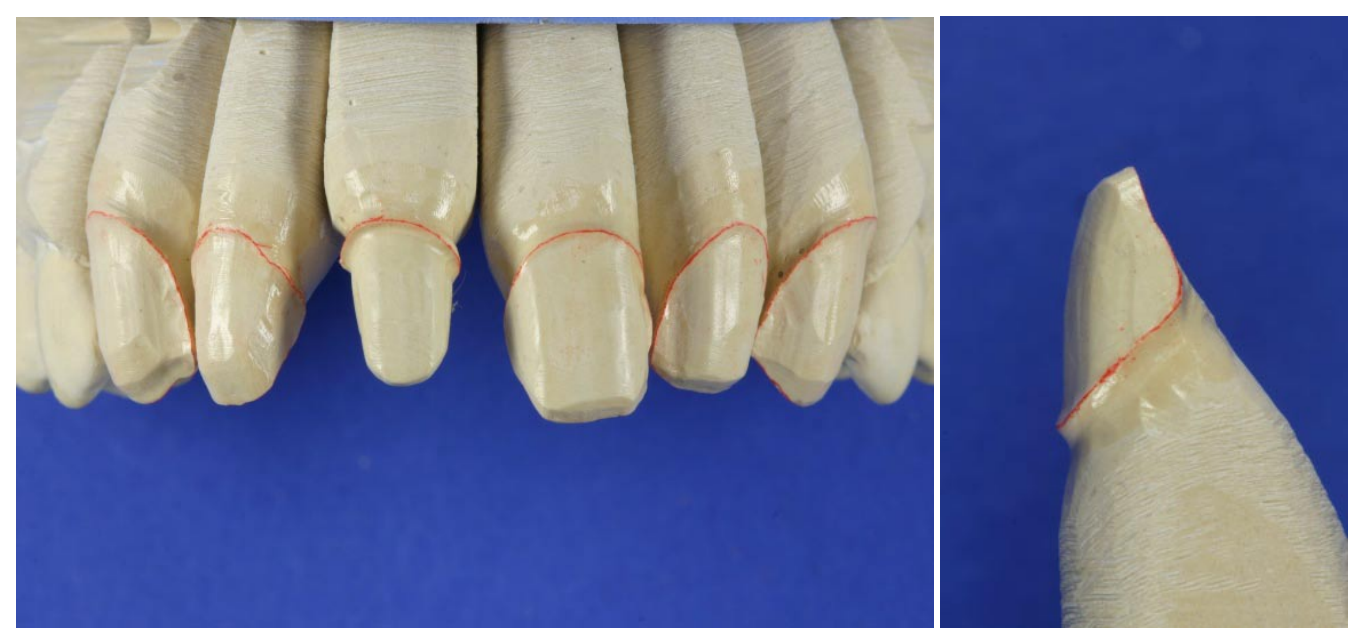

Abb.2: Präparationen von Dr. med. dent. Rinke für Overlap-Veneers

\subsubsection{Keramik}

Die 130 Veneerrestaurationen wurden alle aus Presskeramik (Cergo, DeguDent GmbH, Hanau/Deutschland) hergestellt. Diese lässt sich wie folgt beschreiben:

Cergo ist eine Press-Vollkeramik für ästhetische Restaurationen wie auch zum Beispiel Veneers. Sie hat laut Herstellerangaben neben den guten ästhetischen Eigenschaften die Vorteile der naturnahen Oberflächenhärte mit einem zahnähnlichen Abrasionsverhalten, einer guten Biokompatibilität sowie einer hohen Temperaturwechselbeständigkeit.

Indikationen:

- Inlays, Onlays, Veneers und Kronen

- kann optional mit der Verblendkeramik Duceragold oder Duceragold Kiss verblendet werden.

Kontraindikation:

- Herstellung von Brücken

- tiefe subgingivale Präparationen

- Bruxismus.

Technische Daten:

- chemische Zusammensetzung: $\mathrm{K}_{2} \mathrm{O} \mathrm{Al} \mathrm{Al}_{2} \mathrm{O}_{3} \mathrm{SiO}_{2} \mathrm{Na}_{2} \mathrm{O}$

- Bruchfestigkeit: 100 -120 MPa

- Hydrolysebeständigkeit: Gewichtsverlust $<100 \mu \mathrm{g} / \mathrm{cm}^{2}$

- Presstemperatur: $980^{\circ} \mathrm{C}$

- Vickershärte: 470 - 530 HV02. 


\section{Befestigung:}

Cergo muss adhäsiv befestigt werden. Neben einer besseren Haftung wird so auch eine bessere ästhetische Wirkung erzielt.

Sämtliche Zementierungsflächen der Restaurationen sind für 4 min mit 5\%iger Fluss-Säure zu ätzen.

Alle mit Flusssäure behandelten Flächen sind anschließend zu silanisieren.

Im weiteren Verlauf sind das Adhäsiv und der Zement aufzutragen.

\subsubsection{Adhäsivsystem und Befestigungszement}

\subsection{Adhäsivsystem}

Alle Veneers wurden unter Kofferdam adhäsiv befestigt. Als Adhäsiv wurde Optibond FL (Kerr Hawe, Karlsruhe/Deutschland) ausgewählt.

OptiBond FL ist ein aus zwei Komponenten/zwei Flaschen bestehendes lichthärtendes Primer-Adhäsivsystem. Es gehört zur Gruppe der „total-etch-Systeme“. Bestandteile sind zu 48\% 0,6- $\mu \mathrm{m}$-Barium-Glas-Partikel. Des Weiteren sind die Fluoridabgabe sowie die Röntgenopazität zu erwähnen. Da es ein gefülltes Adhäsivsystem ist, bietet es laut Herstellerangaben gegenüber nicht gefüllten Systemen den Vorteil des Schutzes gegenüber der Mikrospaltbildung bei gleichzeitig guter Adhäsion an den verschiedenen Oberflächen. Das Lösungsmittel für die einzelnen Substanzen ist Ethanol.

\subsection{Befestigungszement}

Die Auswahl der Zemente wurde nach Zufall auf die Patienten verteilt.

Bei Versorgungen mit hochästhetischen, relativ transparenten Materialien, wie auch hier bei Veneers, ist die Farbannahme der Restauration von benachbarten Zähnen möglich (Chamäleoneffekt). Um diese Farbwirkung zu ermöglichen, ist ein relativ transparenter Zement Voraussetzung. Diesen Anforderungen entsprechen die in den folgenden Kapiteln beschriebenen Zemente. 


\subsection{Calibra}

Calibra ${ }^{\circledR}$ (Dentsply DeTrey, Konstanz/Deutschland) ist ein lichthärtendes, dualhärtendes oder selbsthärtendes Harzkomposit, das den Vorteil der Aushärtung auch bei starker Opazität bietet.

Weiterer Vorteil ist das Vorhandensein zweier verschiedener Katalysatorpasten. Diese unterscheiden sich in ihrer Viskosität. Bei Verwendung der visköseren der beiden wird das Fließen der Masse in ungewünschte Bereiche vermieden wobei die niedrigviskösere Paste gut unter Kronen und Brücken verteilt werden kann, um so einen gleichmäßigen Zementfilm zu schaffen. Des Weiteren ist die sehr kleine Körnung von 12-18 $\mu \mathrm{m}$ zu erwähnen, welche laut Herstellerangaben eine der Grundvoraussetzungen für einen geringen Randspalt ist.

\section{Zusammensetzung:}

Grundlage: Dimethacrylat Harze, Kampferquinone, Photoinitiator, Stabilisatoren, Glasfüller, Fumed Silika, Titanium Dioxide, Pigmente.

Katalysator: Dimethacrylat Harze, Stabilisatoren; Glasfüller; Fumed Silika.

Bindemittel: Aceton, Ethylalkohol, Organo Silane

\subsection{Variolink}

Bei Variolink (Ivoclar Vivadent, Schaan/Liechtenstein) handelt es sich um ein dualhärtendes (licht- und selbsthärtend) Befestigungskomposit-System zur adhäsiven Befestigung von Keramik- und Kompositrestaurationen. Zur Verwendung bei Veneers kann es ebenfalls als nur lichthärtendes Material eingesetzt werden, wobei nur die Base eingesetzt wird. Laut Herstellerangaben ist die spezielle Füllerzusammensetzung für gute physikalische Eigenschaften, hohe Abrasionsresistenz, hohe Röntgenopazität, gute optische Eigenschaften sowie eine kontinuierliche Fluoridfreisetzung verantwortlich.

Bei einem Mischverhältnis Basispaste zu Katalysator von 1:1 liegt die Verarbeitungszeit laut Hersteller bei $37^{\circ} \mathrm{C}$ in 3-4 Minuten. 
Zusammensetzung:

Variolink besteht zum einen aus einer Monomatrix mit Bis-GMA, Urethandimethacrylat und Triethylenglycoldimethacrylat. Ein weiterer Bestandteil sind die anorganischen Füllstoffe aus Bariumglas, Ytterbiumtrifluorid, Ba-Al-Fluorsilikatglas und sphäroidem Mischoxid.

Des Weiteren sind Katalysatoren, Stabilisatoren und Pigmente enthalten. Die Partikelgröße liegt zwischen 0.04-3.0 $\mu \mathrm{m}$ (mittlere Partikelgröße 0,7 $\mu \mathrm{m}$ ).

\subsection{Indikationen und Kontraindikationen}

Indikationen:

- adhäsive Zementierung von Keramik-und Komposit-Restaurationen

- adhäsive Zementierung von Metall-Restaurationen

- adhäsive Zementierung von VMK- (Vita-Metall-Keramik), -kronen, -brücken.

- adhäsive Zementierung von Stiften.

Kontraindikationen:

- Allergie gegen Bestandteile des Befestigungszementes

- keine Trockenlegung möglich

- zuvor verwendete phenolische Substanzen (z.B. Eugenol), da sie die Polymerisation inhibieren.

\subsection{Arbeitsschritte}

Folgende Schritte werden bei der Applikation durchgeführt:

1. Präparierten Bereich isolieren und säubern (Bimsstein)

2. Überprüfen der Passform und der Ästhetik der Restauration (Try-in Paste)

3. Trockenlegung

4. Schmelz- und Dentinkonditionierung (35\%ige Phosphorsäure für 15-20 Sekunden)

5. geätzte Bereiche für 30 Sekunden mit Wasserspray absprühen und trocknen

6. OptiBond FL Primer auftragen und verblasen

7. OptiBond FL-Adhäsiv einmassieren und lichthärten

8. Behandlung der Keramikrestauration 
9. Ätzen der Keramikinnenfläche mit 5\%iger Flusssäure (Vita Keramikätzgel, Vita Zahnfabrik, Bad Säckingen/Deutschland) für 60-90 Sekunden

10. Säubern der Flächen

11. Silansierung der Veneerinnenfläche mit Monobond S (Ivoclar Vivadent, Schaan/Liechtenstein oder Calibra Silane)

12. Komposit auftragen (Calibra oder Variolink)

13. Platzieren des Veneers

14. Entfernen des überschüssigen Materials

15. Lichthärten (120 Sekunden)

16. Okklusion überprüfen (Front-Eckzahnführung)

17. Oberflächenbehandlung und Politur.

\subsection{Methoden}

\subsubsection{Klinische Untersuchungen}

Die klinische Nachuntersuchung der Patienten mit Veneerrestaurationen erfolgte zwischen 2008 und 2009 nach Überprüfung der Ein- und Ausschlusskriterien. Der Untersucher hatte keine Kenntnis über den verwendeten Befestigungszement bei den einzelnen Veneers („Verblindung“ des Behandlers). Die Reihenfolge der Patienten war willkürlich und lediglich von der Terminabsprache abhängig. Der Untersuchungszeitpunkt war etwa 36 Monate nach Insertion der Restaurationen. Es wurden eine zahnärztliche Untersuchung der Veneers mit Sonde, Spiegel und Zahnseide durchgeführt. Die Vitalität wurde mit einem $\mathrm{CO}_{2}$ - Kältetest überprüft. Anschließend wurden intraorale Fotoaufnahmen (s. Abb. 4) angefertigt. Alle Veneers wurden hinsichtlich unterschiedlicher Kriterien wie Verlust, Frakturen, Karies, Lockerung, Randschluss und marginaler Verfärbungen untersucht. Dabei wurden die Veneers in situ auf Randdichtigkeit, Randverfärbung, Sekundärkaries, Farbunterschied zu den Nachbarzähnen und anatomische Form überprüft.

Für die Untersuchung eines Probanden war ein Zeitbedarf von 10-15 Minuten eingeplant. Es bestanden bei der Untersuchung keine Risiken und die Probanden erhielten keine finanzielle Entschädigung für die Teilnahme an der Untersuchung. 
Zudem erfolgte eine Auswertung der Akten hinsichtlich der persönlichen Daten, wie Geschlecht und Alter der Patienten. Außerdem wurde anhand der Dokumentationsbögen in den Patientenkarteien festgestellt, ob im Zeitraum bis zur Nachuntersuchung negative Ereignisse, wie Rezementierung, endodontische Behandlung, biologische Komplikationen oder Verlust aufgetreten sind, und gegebenenfalls in die Studie mit aufgenommen.

Ein Beispiel für eine in dieser Studie untersuchte Veneerversorgung der gesamten Oberkieferfront ist in Abbildung $3 \mathrm{zu}$ sehen. Bei diesen Restaurationen ist der perfekte Randschluss zu erkennen. Außerdem liegen keine marginalen Verfärbungen, Anzeichen von Frakturen oder Sekundärkaries vor.

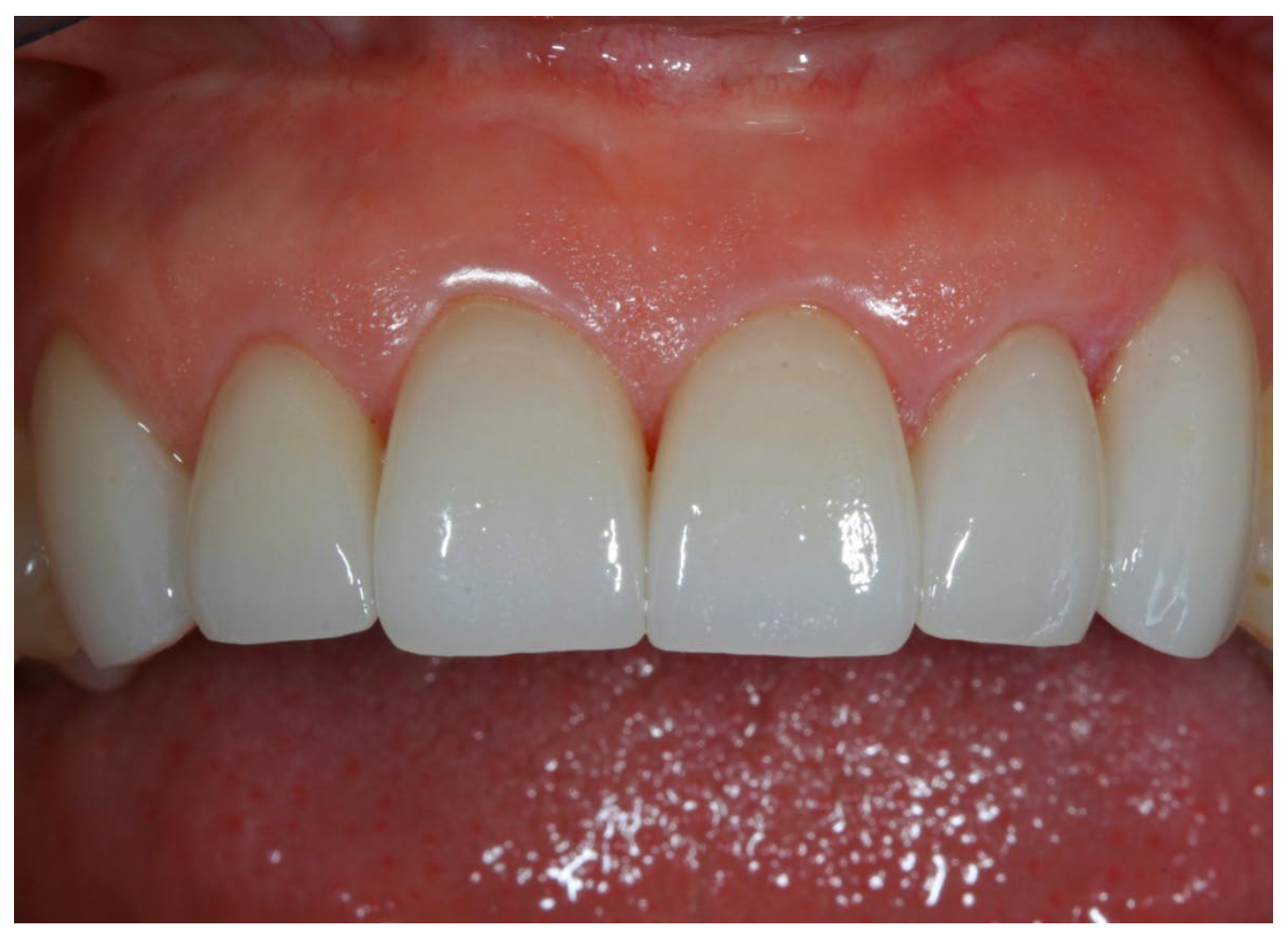

Abb. 3: Beispiel einer in der Studie untersuchten Veneerversorgung

(Randschluss optimal, Gingiva entzündungsfrei, approximaler Kontakt vorhanden, Farbe harmonisch)

\subsubsection{Statistische Auswertung der Ergebnisse}

Die statistische Auswertung erfolgte zusammen mit der Abteilung Medizinische Statistik der Universitätsmedizin Göttingen (Direktor: Prof. Dr. rer. nat. Brunner).

Untersucht wird die Haltbarkeit der Veneers. 
Als Maßstab für die Haltbarkeit wird hierzu verwendet:

1. die Verlustrate - d. h. wie viel Prozent der Veneers waren noch vorhanden, also in situ. Dabei spielt es keine Rolle, ob Komplikationen wie Wurzelkanalbehandlung, Rezementierung etc. aufgetreten waren.

Die Zeit war hierbei definiert, als Zeitraum zwischen der Zementierung und dem letzten Kontrolltermin, bzw. bei Verlust bis zum vorherigen Kontrolltermin, wie er in der Patientenakte dokumentiert war.

2. die Erfolgsrate - d.h. wie viel Prozent der Veneers waren noch vorhanden und wiesen gleichzeitig keine Komplikationen auf (Verluste + ggf. erforderliche Nachbehandlungen).

Als mögliche Kovariablen, d. h. Einflussgrößen, wurden der verwendete Zement sowie die Kieferposition des Veneers (Oberkiefer oder Unterkiefer) mit einbezogen.

Die verschiedenen Beobachtungen am gleichen Patienten (mehrere Veneers pro Patient) sind voneinander abhängig. Dieser Abhängigkeit wurde durch eine adjustierte Varianzschätzung im Cox Regressionsmodell (time-to-event-Analyse) Rechnung getragen. Zudem wurden Ereigniszeitanalysen durchgeführt. Dabei wurde die Zeit bis zum Eintreten eines bestimmten Ereignisses ("time to event") zwischen zwei oder mehr Gruppen verglichen, um die Wirkung von prognostischen Faktoren, medizinischer Behandlung oder schädlichen Einflüssen zu schätzen. Das Ereignis konnte dabei der Verlust sein, jedoch auch andere Ereignisse, wie eine Rezementierung, Wurzelkanalbehandlung, Sekundärkaries oder das Eintreten einer biologischen Komplikation.

Zur Analyse der Daten wurde somit ein marginales Modell verwendet (GERDS et al. 2009). Dabei wurde für jeden Einflussfaktor eine univariate Cox-Regression durchgeführt.

Ein Signifikanzniveau < 5\% wurde zur Bestimmung des statistisch signifikanten Einflusses akzeptiert.

Die statistische Analyse wurde mit der freien Software R (SAS 9.2, SAS Institute Inc., Cary, NC, USA, Version 2.8, www.r-project.org) durchgeführt. 


\section{Ergebnisse}

\subsection{Probanden}

Der Untersuchungszeitpunkt der Veneerrestaurationen lag im Durchschnitt bei 38,6 Monaten (min: 6,2 Monate; max: 84,7 Monate).

Von 41 in Frage kommenden Patienten konnten 37 (90,2\%) in diese Studie einbezogen werden. Hierbei handelte es sich um funktionsdiagnostisch unauffällige Patienten mit kariesfreiem, suffizient versorgtem Gebiss.

Die orale Situation zweier Patienten vor der Therapie mittels Keramikveneers bzw. ca. 36 Monate nach einer erfolgreichen Insertion zeigen beispielhaft die Fotoaufnahmen in den Abbildungen 4 und 5.

a)

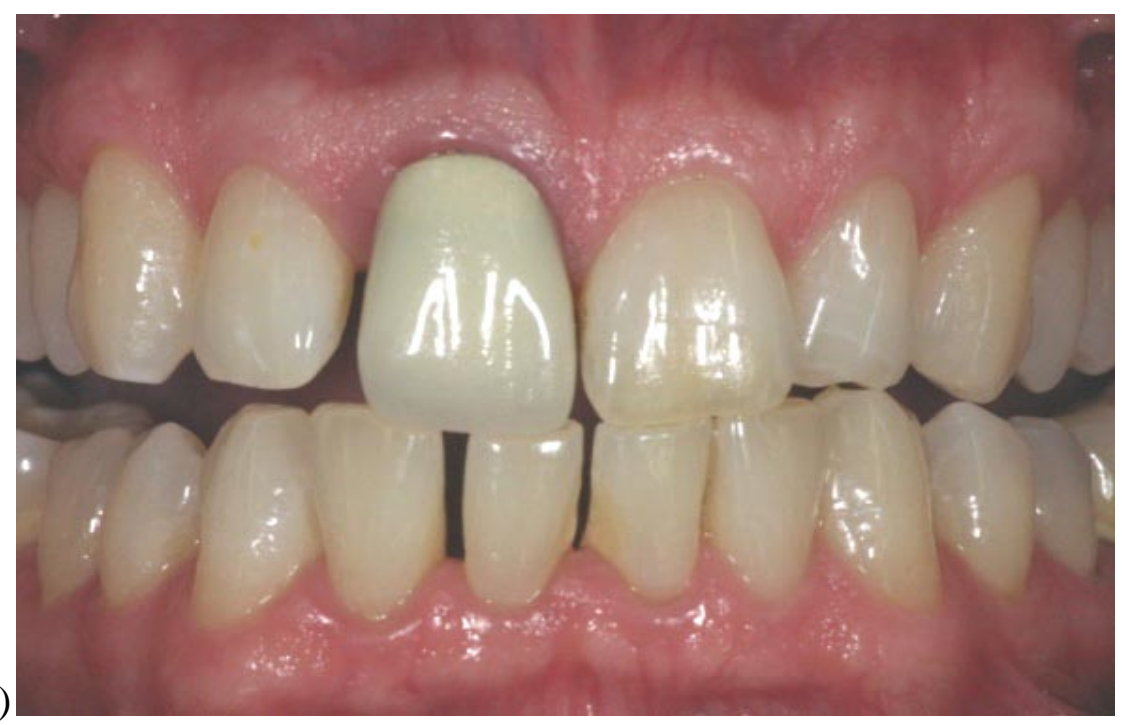

b)

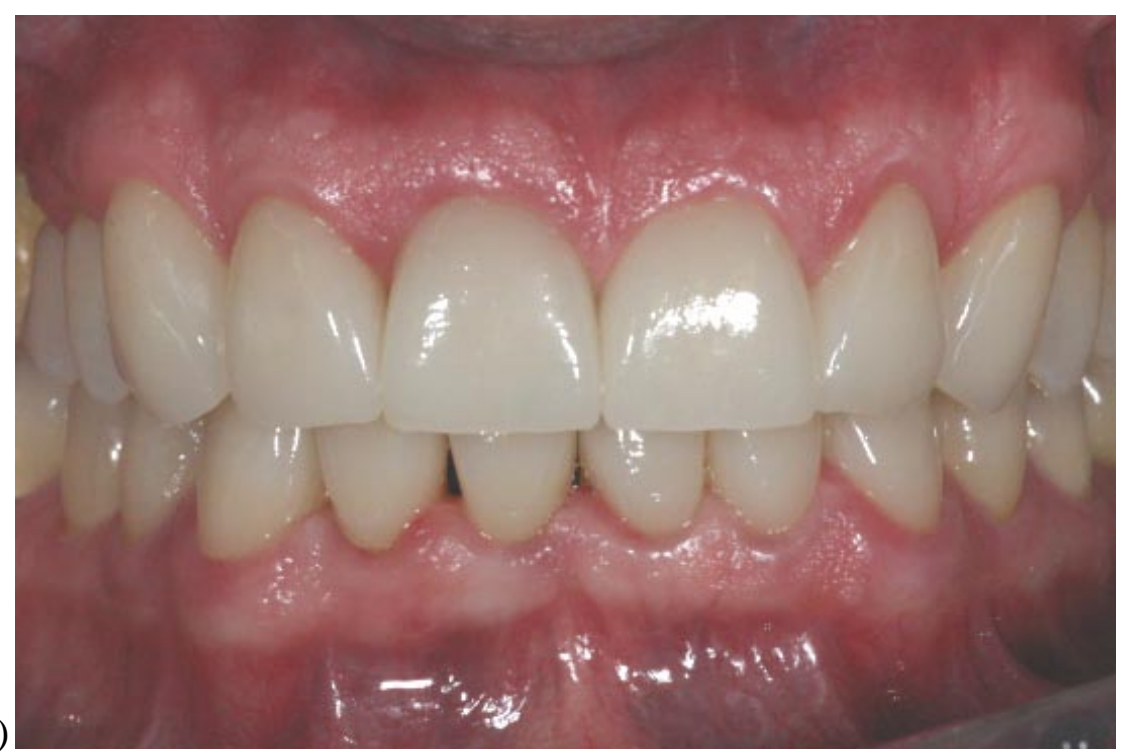

Abb.4: Patientenfall I: vorher (a), nachher (b) 
a)

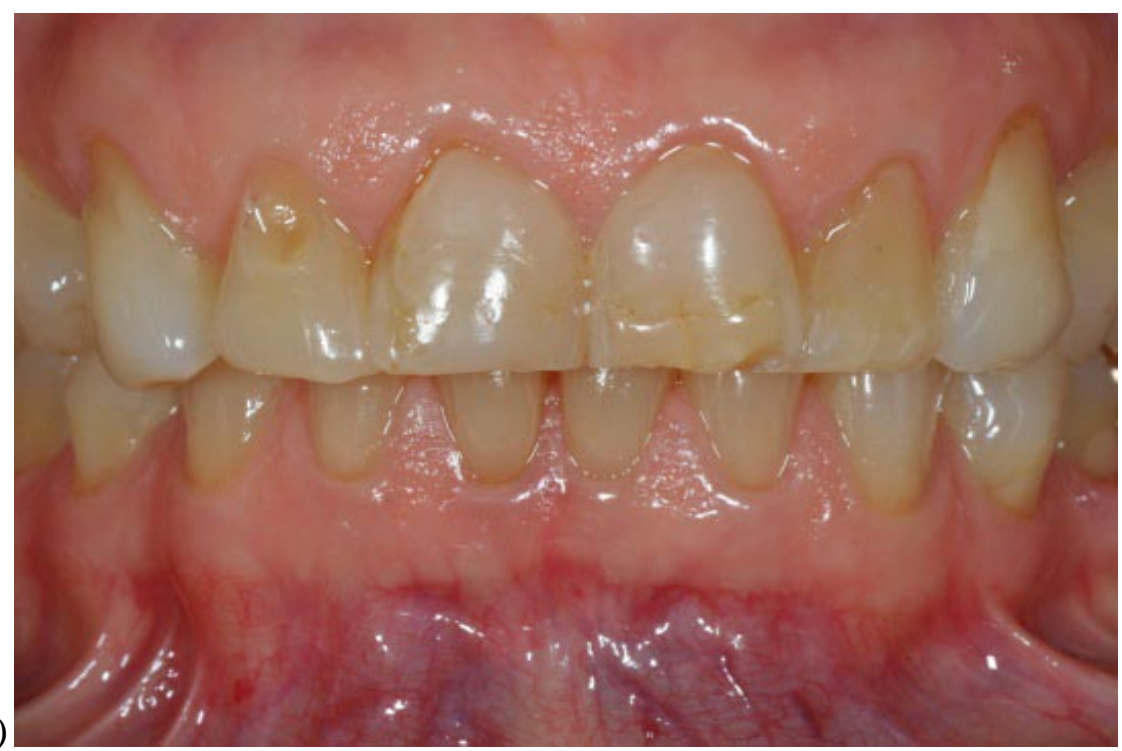

b)

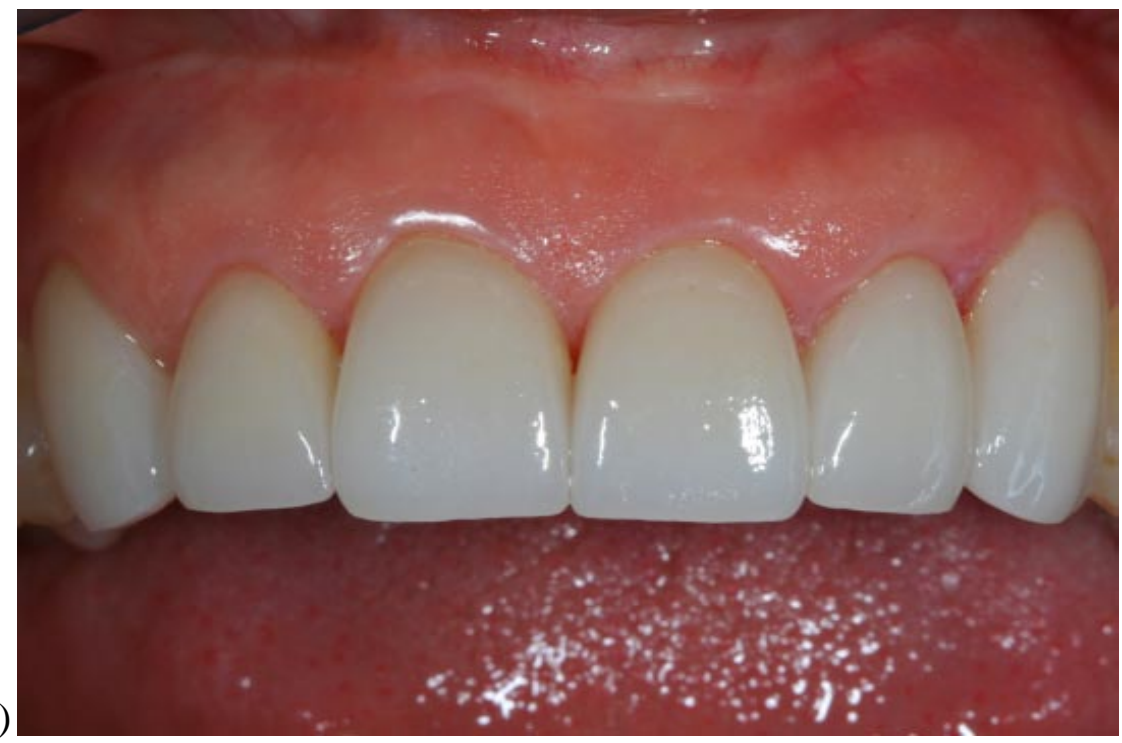

Abb.5: Patientenfall II: vorher (a), nachher (b)

Die Keramikversorgungen verteilten sich auf 21 weibliche und 16 männliche Patienten. Dies entspricht einem Anteil von 56,76\% weiblichen und 44,24\% männlichen Personen (s. Abb.6). 


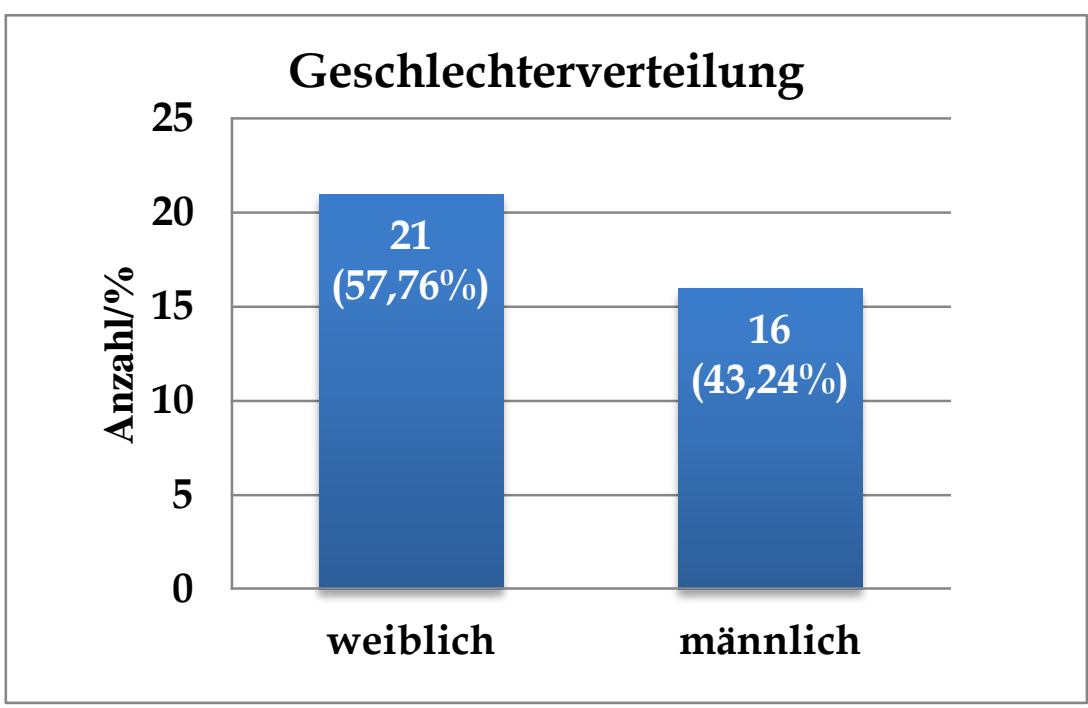

Abb.6: Absolute und relative Verteilung der Patienten auf die Geschlechter

Das Alter der Patienten reichte von 20 bis 73 Jahre. Der Durchschnitt lag bei 49,82 Jahren. Die Standardabweichung im Bezug auf das Alter beträgt 11,85 Jahre.

Die 37 Patienten wurden ihrem Alter entsprechend 10 Jahre umfassenden Gruppen zugeordnet, um die Verteilung der Veneers auf einzelnen Altersstufen zu strukturieren (s. Abb.7). Danach lag der Schwerpunkt der Altersverteilung mit 13 Probanden in der Altersstufe 40-49 Jahre. 50 bzw. über 50 waren 15 Patienten.

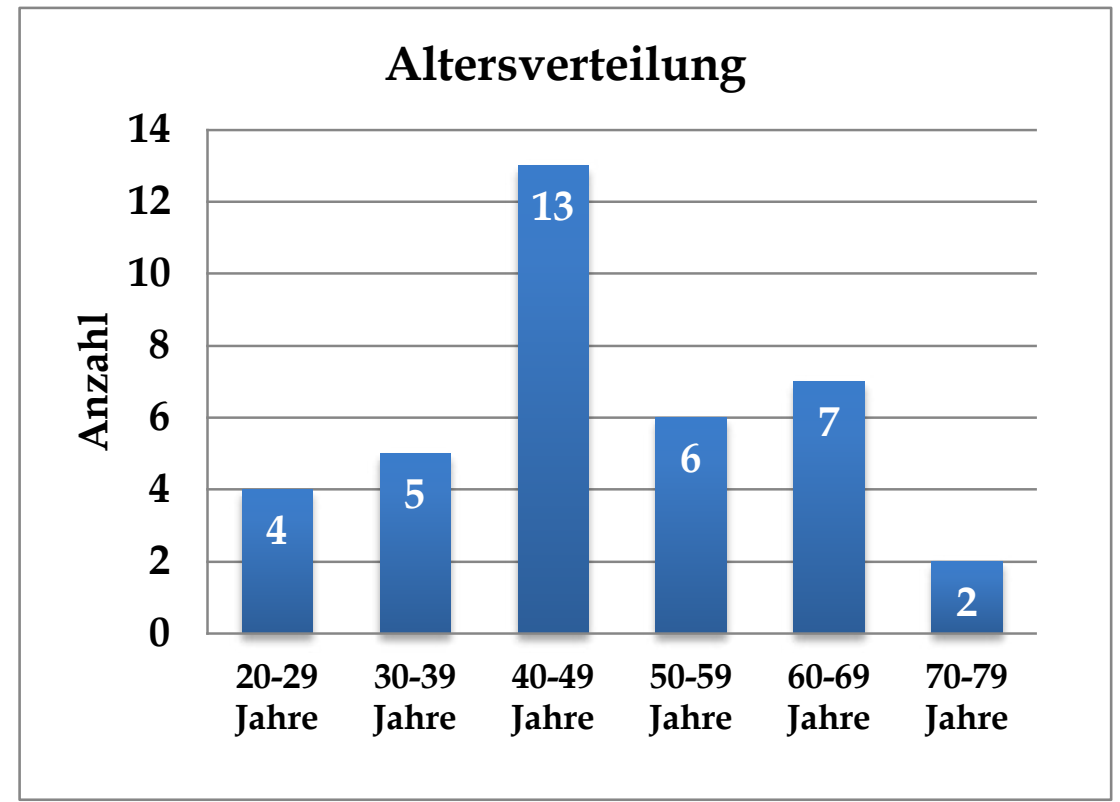

Abb.7: Altersverteilung der Patienten 


\subsection{Veneers}

Insgesamt wurden 130 Zähne vom selben Behandler in der Zahnarztpraxis Dr. med. dent. S. Rinke \& S. Schmidt versorgt, die sich auf 76 Zähne im Oberkiefer und 54 im Unterkiefer verteilten. Aufgrund der zahlreichen Ein- und Ausschlusskriterien war ein leichtes Missverhältnis der Veneers zwischen Ober- und Unterkiefer nicht zu vermeiden (s. Tab.3).

Die Verteilung auf die einzelnen Frontzähne ist Tabelle 3 und Abbildung 8 zu entnehmen.

Tab.3: Veneerverteilung auf die einzelnen Frontzähne (Ober- und Unterkiefer)

\begin{tabular}{|c|c|c|c|c|c|c|}
\hline Zahn & 13 & 12 & 11 & 21 & 22 & 23 \\
\hline Anzahl & 10 & 13 & 16 & 17 & 14 & 6 \\
\hline Anzahl & 9 & 10 & 9 & 9 & 9 & 8 \\
\hline Zahn & $\mathbf{4 3}$ & $\mathbf{4 2}$ & $\mathbf{4 1}$ & 31 & 32 & 33 \\
\hline
\end{tabular}

Oberkiefer: $n=76 \quad$ Unterkiefer: $n=54$ gesamt: $N=130$

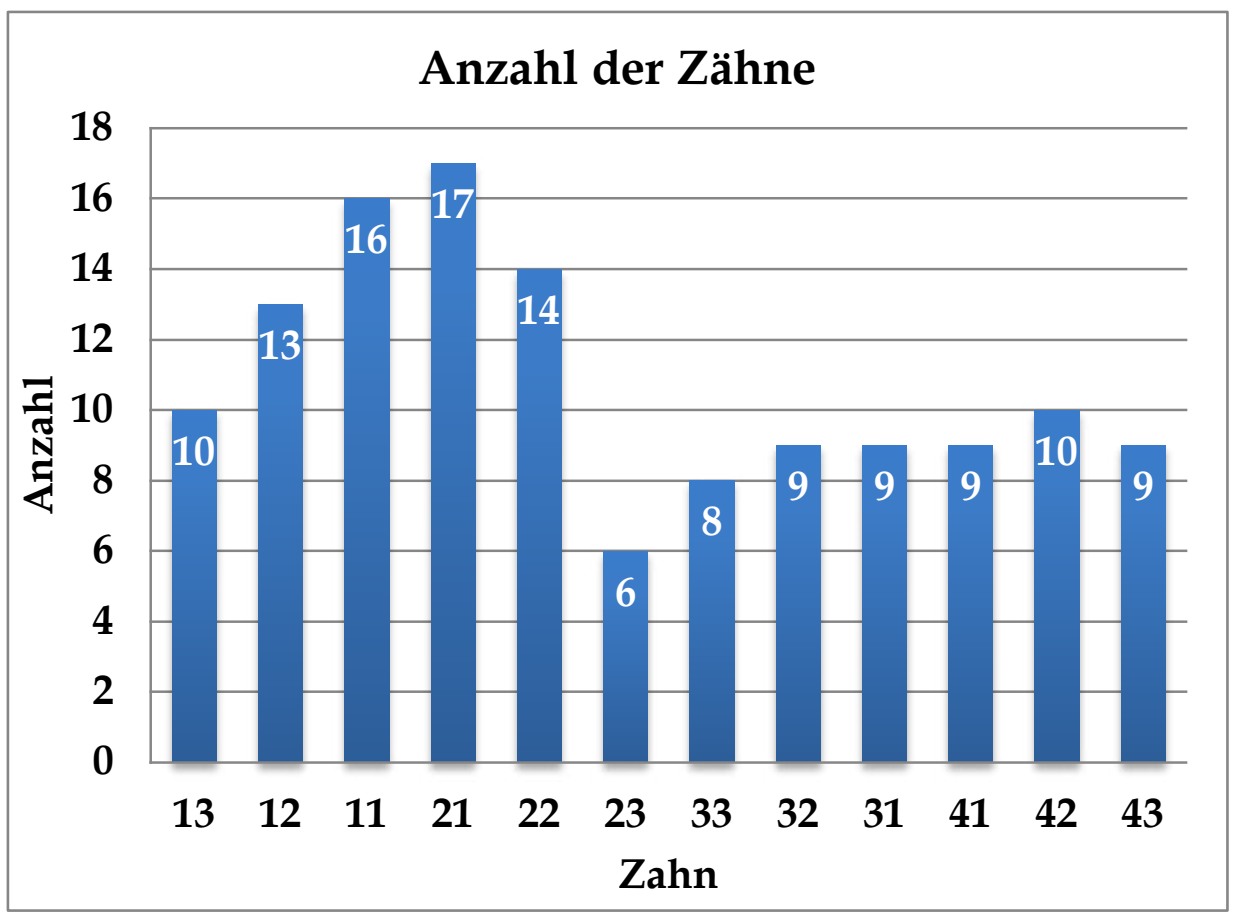

Abb.8: Veneerverteilung auf die einzelnen Frontzähne (Ober- und Unterkiefer) 
Bei Betrachtung der Befestigungszemente ist zu erkennen, dass bei 59 Veneers Variolink (Ivoclar Vivadent, Schaan/Liechtenstein) (45\%) und bei 71 Veneers Calibra (Dentsply, Konstanz/Deutschland) (55\%) verwendet wurde.

Hieraus folgt die nicht gleichmäßige Verteilung der Veneers, wie sie auch in Abbildung 9 abzulesen ist.

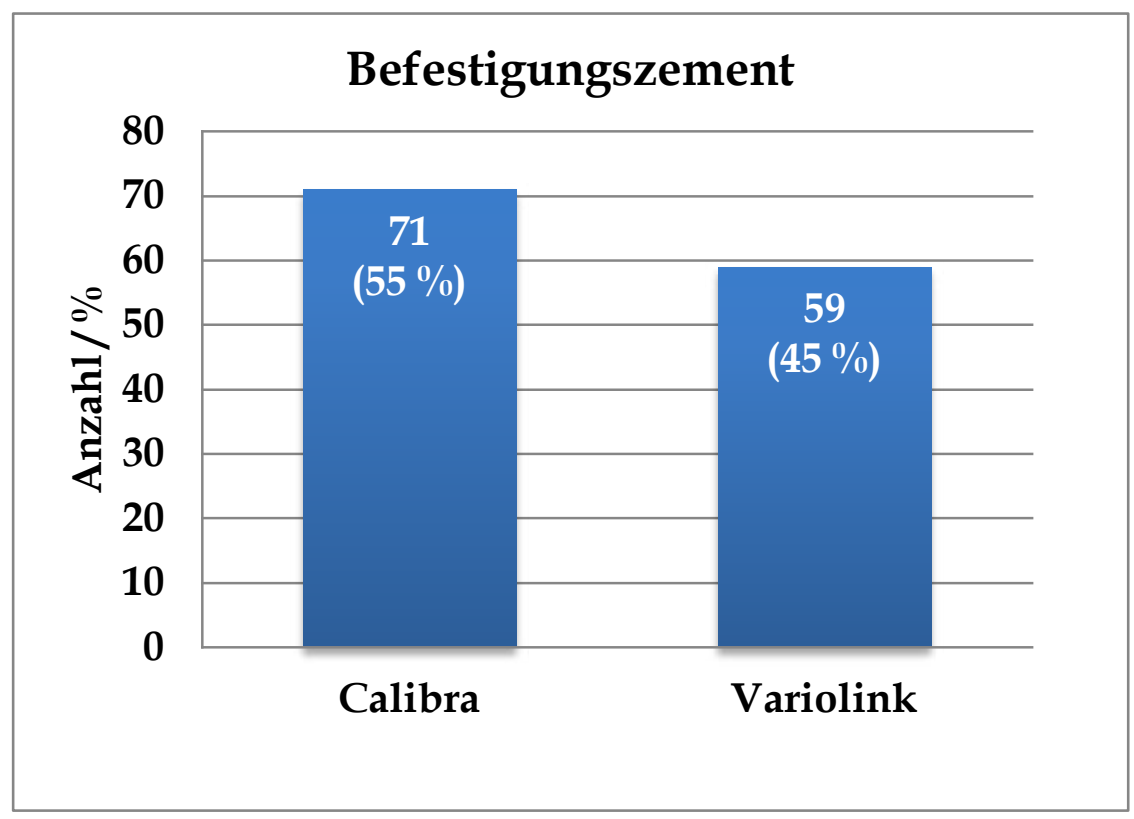

Abb.9: Verteilung der Zemente auf die Veneers

Die Verteilung der einzelnen Befestigungszemente auf die einzelnen Kiefer ist in Tabelle 4 dargestellt. Auch hier besteht aufgrund der Ein- und Ausschlusskriterien ein Missverhältnis im Bezug auf die Veneerverteilung. Im Oberkiefer überwog der Einsatz des Befestigungszements Variolink, im Unterkiefer der von Calibra.

Tab. 4: Anzahl (n) der Veneerverteilung im Zusammenhang der Region zum Befestigungszement

\begin{tabular}{|c|c|c|}
\hline & Oberkiefer & Unterkiefer \\
\hline Calibra & $\mathrm{n}=34$ & $\mathrm{n}=37$ \\
\hline Variolink & $\mathrm{n}=42$ & $\mathrm{n}=17$ \\
\hline
\end{tabular}




\subsection{Verlust- und Erfolgsrate}

Mit den Untersuchungsergebnissen wurden die Überlebens- bzw. Erfolgsraten der Veneerrestaurationen geschätzt. Hierzu wurde der Kaplan-Meier-Schätzer verwandt, mit dem sich die Wahrscheinlichkeit schätzen lässt, dass bei einem Versuchsobjekt, in diesem Fall das Veneer, ein bestimmtes Ereignis innerhalb eines Zeitintervalls, hier Monate, nicht eintritt. Die Ergebnisse der biometrischen Auswertungen nach 36 Monaten werden im Folgenden tabellarisch und graphisch vorgestellt. Dabei sind in den Tabellen die mittleren Überlebenszeiten in Monaten und die Überlebenswahrscheinlichkeiten in Prozent angegeben. In den Abbildungen ist auf der Ordinate (yAchse) der überlebende bzw. ereignislose Anteil der beobachteten Veneers, auf der Abszisse (x-Achse) die Zeit in Monaten in einem linearen Maßstab aufgetragen.

\subsubsection{Verlustrate}

Die Analyse ergab, dass zwei Zeiträume vorliegen, in denen die Verlustrate im Verhältnis zur übrigen Zeit hoch ist. Bei der Untersuchung nach 36 Monaten waren insgesamt fünf Verluste zu verzeichnen. In den ersten 21 Monaten waren vier Verluste aufgetreten, ein weiterer wurde nach etwa 48 Monaten festgestellt. Demnach lassen sich folgende Abfälle der Kurve (s. Abb.10) beschreiben:

Nach 13,31 Monaten fällt die Erfolgsrate von 100\% auf 98,89\%, nach 15,48 Monaten auf 97,7\%, nach 19,51 Monaten auf 96,46\%, nach 20,69 Monaten auf 95,12\%, um nach 47,93 Monaten auf 90,99\% zu sinken (s. Tab.5).

Vier der genannten Verluste sind auf Keramikfrakturen (s. Abb.11) zurückzuführen und ein Verlust hatte den totalen Zahnverlust im Sinne einer Extraktion als Ursache.

Tab.5: Überlebensanalyse, Überlebenszeit: T(m), Überlebenswahrscheinlichkeit: \%, Art der Verluste

\begin{tabular}{|c|c|c|}
\hline Überlebenszeit & $\begin{array}{c}\text { Überlebens- } \\
\text { wahrscheinlichkeit } \%\end{array}$ & Art der Verluste \\
\hline 13,31 & 98,89 & Fraktur \\
\hline 15,48 & 97,7 & Extraktion \\
\hline 19,51 & 96,46 & Fraktur \\
\hline 20,69 & 95,12 & Fraktur \\
\hline 47,93 & 90,99 & Fraktur \\
\hline
\end{tabular}




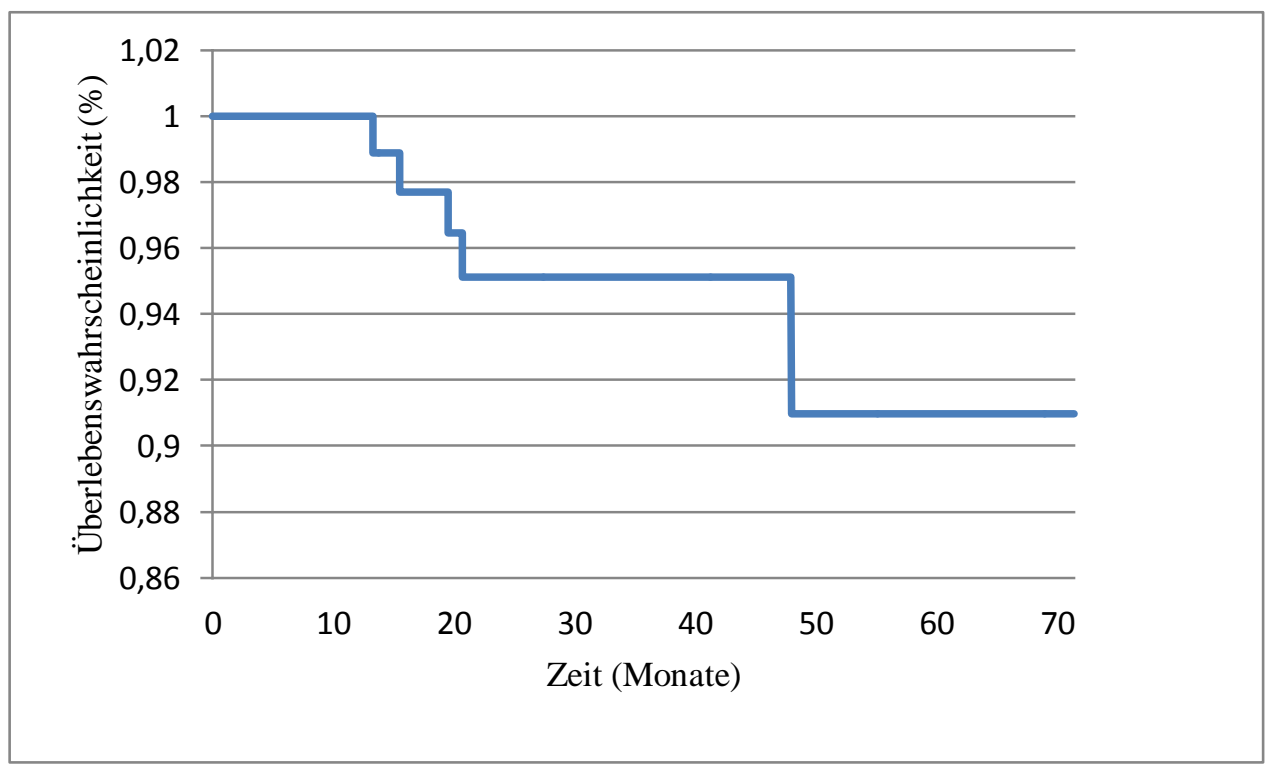

Abb.10: Überlebensanalyse in Abhängigkeit von der Zeit

Drei der vier Frakturen sind in Abbildung 11 fotographisch festgehalten. Im oberen Foto (Abb. 11a) ist ein frakturiertes Oberkiefer-Keramikveneer am Zahn 22 zu sehen. Die Fraktur verläuft längs der Zahnachse. Die Präparation wurde bis in das Dentin ausgedehnt. Im mittleren Foto (Abb. 11 b) ist eine inzisale Längsfraktur des Veneers am Zahn 42 zu erkennen. Das untere Foto in der Abbildung 11 c zeigt eine zervikale Keramikabplatzung am Zahn 12 sowie einen Längsriss in der Keramik. In diesen Fällen konnte die Restauration nicht erhalten werden. 
a)

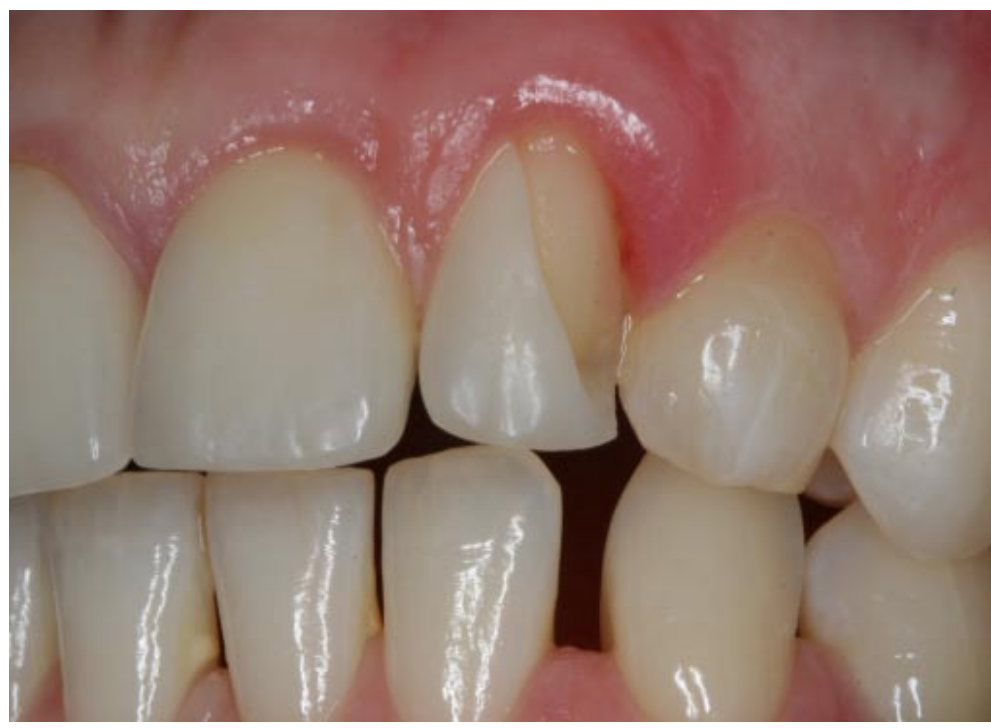

b)

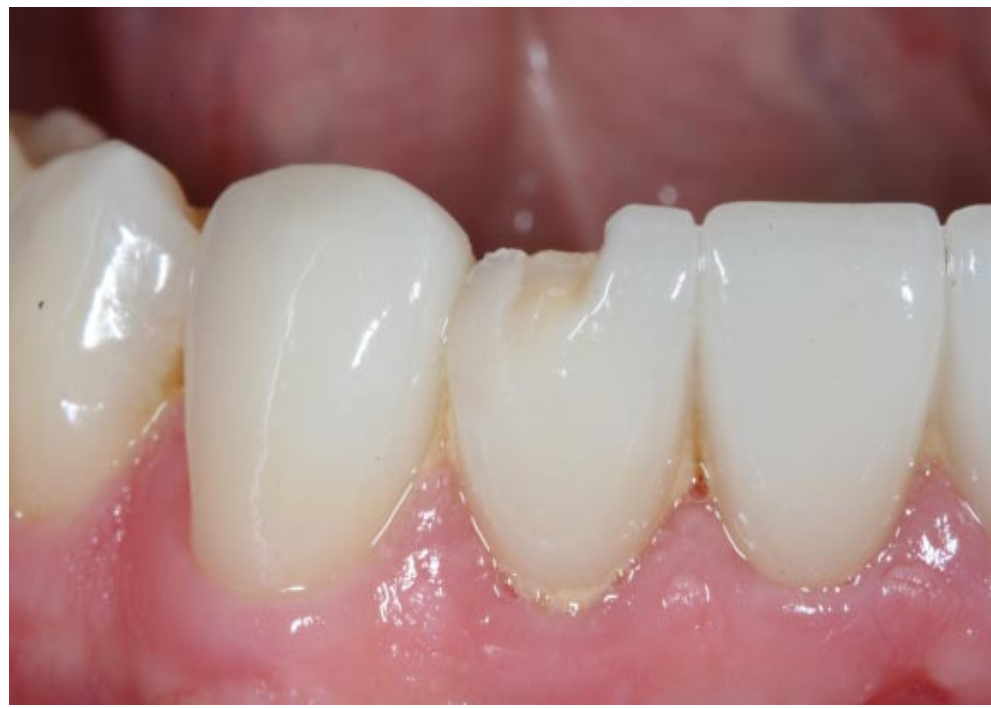

c)

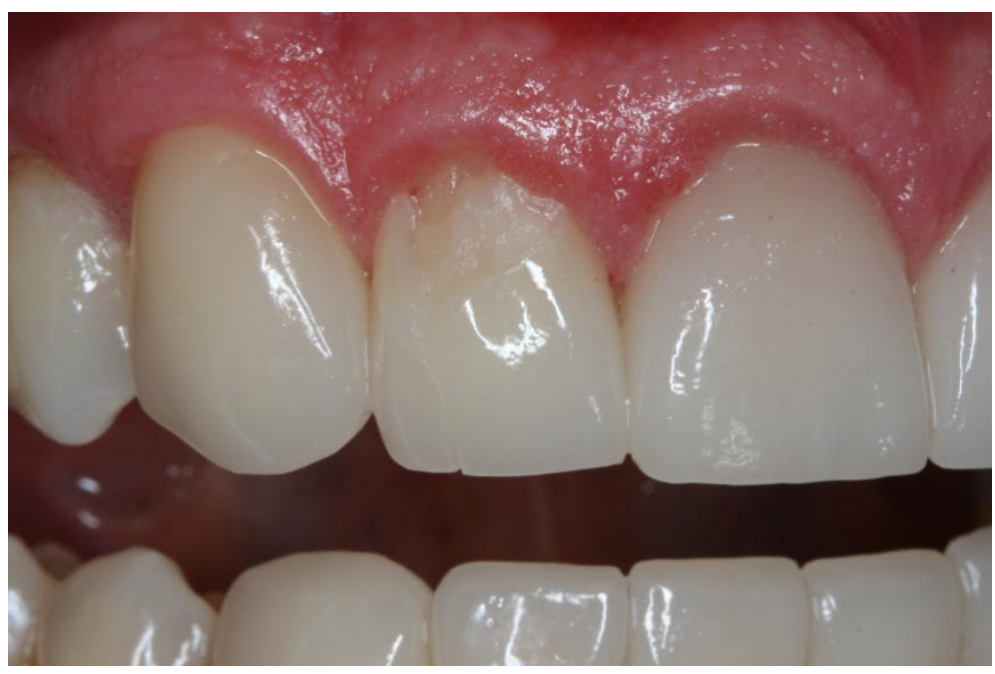

Abb.11: Fallbeispiele für Keramikfrakturen:
a) Oberkieferveneer (Zahn 22)
b) Unterkieferveneer (Zahn 42)
c) Oberkieferveneer (Zahn 12) 


\subsubsection{Faktor Zement}

Der Unterschied im Bezug auf das geschätzte Risiko beim Einsatz der Befestigungszemente Variolink und Calibra wurde mit dem Wert des Hazard-Ratio geschätzt. Die Wahrscheinlichkeit ein mit Variolink befestigtes Veneer zu verlieren, ist nach 36 Monaten um den Faktor 2,13 höher als im Vergleich zu Calibra, was im Verlauf der beiden Kurven in Abbildung 12 deutlich zu erkennen ist. Dieser Risikofaktor $(=2,13)$ ist nicht signifikant von 1 verschieden, da $\mathrm{p}=0,2$ ist. Signifikant sind erst Unterschiede, bei denen p kleiner 0,05 (5\%) ist.

Beim Zement Variolink kommt es zu vier Verlusten. Nach 13,31 Monaten sinkt der Prozentsatz von 100\% auf 97,96\% und nach 15,48 Monaten von 97,96\% auf 95,92\%. Der größte Unterschied zwischen den Verlustraten der Veneers beginnt nach 20,69 Monaten und steigt nach etwa 47,93 Monaten noch weiter an.

Nach 20,69 Monaten sinkt der Wert um weitere ca. 2\% auf 93,88\% und nach ca. 48 Monaten auf 89,61\%.

Die Anzahl der mit Calibra befestigten Veneers bleibt hingegen relativ stabil. Hier ist lediglich ein Verlust nach 19,51 Monaten zu erkennen, der einen Abfall von 100\% auf 96,88\% zufolge hat. Danach bleibt die Anzahl der mit Calibra befestigten Veneers konstant (Tab.6).

Tab.6: Überlebensanalyse bezogen auf die Befestigungszemente, Überlebenszeit: T(m),

Überlebenswahrscheinlichkeit: \%

\begin{tabular}{|c|c|c|}
\hline \multirow{2}{*}{ Überlebenszeit } & \multicolumn{2}{|c|}{$\begin{array}{c}\text { Überlebens- } \\
\text { wahrscheinlichkeit \% }\end{array}$} \\
\cline { 2 - 3 } & Calibra & Variolink \\
\hline 13,31 & & 97,96 \\
\hline 15,48 & & 95,92 \\
\hline 19,51 & 96,88 & \\
\hline 20,69 & & 93,88 \\
\hline 47,93 & & 89,61 \\
\hline
\end{tabular}




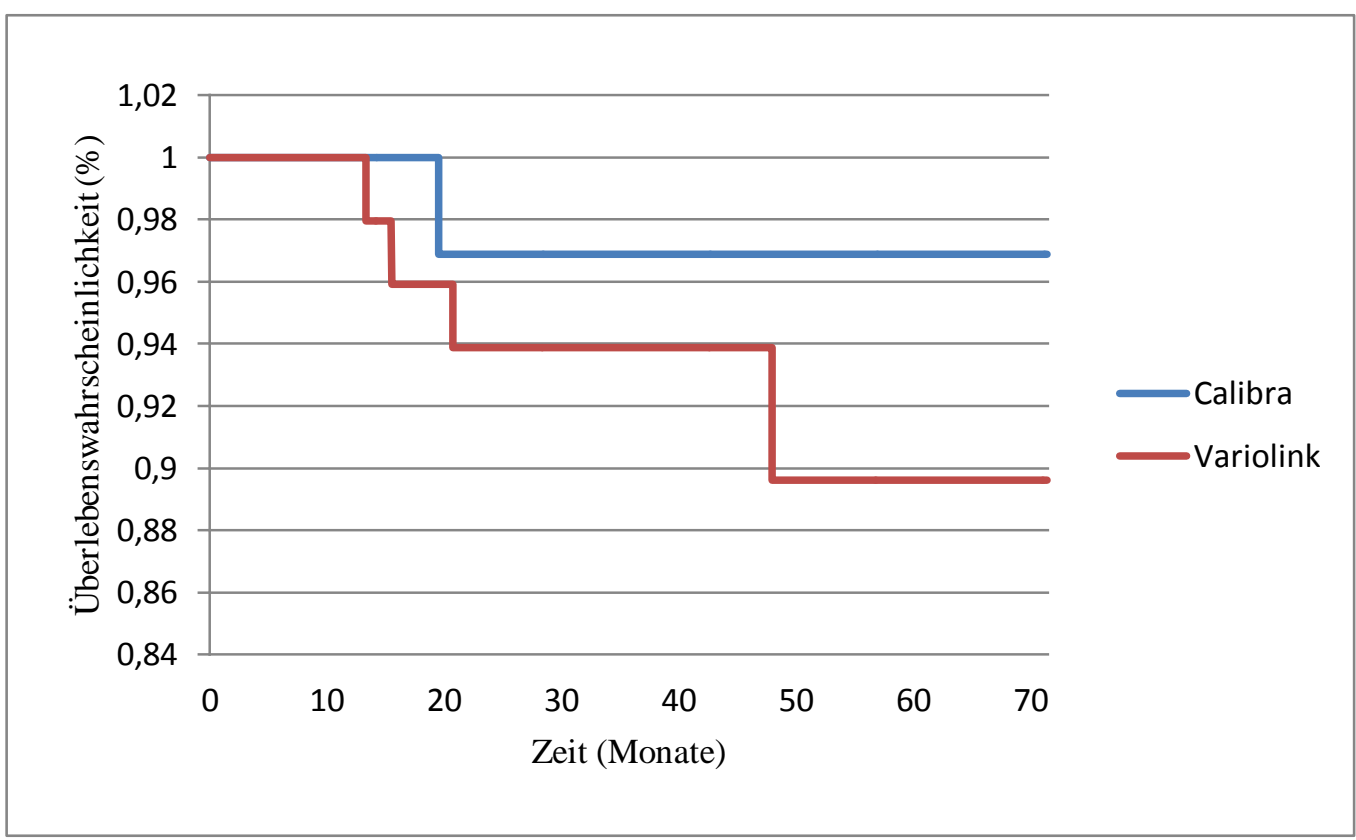

Abb.12: Überlebensanalyse bezogen auf die Befestigungszemente in Abhängigkeit von der Zeit

\subsubsection{Faktor Region}

Im Bezug auf die Region, d.h. Ober- und Unterkiefer, lässt sich feststellen, dass das Risiko des Verlustes für den Oberkiefer nach 36 Monaten um den Faktor 1,82 (1/0,548, d.h. Kehrwert des Unterkiefer-Hazard-Ratio-Wertes) höher liegt bzw. der Unterkiefer weist ein um den Faktor 0,548 stabileres Veneer auf. Auch hier besteht keine Signifikanz, da $\mathrm{p}=0,3$ ist.

Im Unterkiefer kam es im Beobachtungszeitraum von 36 Monaten nach 19,51 Monaten zu einem Verlust eines Veneers. Hier sinkt die Kurve von 100\% auf 96,77\%.

Im Oberkiefer sind vier Verluste aufgetreten, die in der zeitlichen Abfolge denen der mit Variolink befestigten Veneers entsprechen. Demzufolge kam es nach 13,31 Monaten zum ersten Verlust, welcher zu einem Sinken der Kurve von 100\% auf 98,11\% führt, nach 15,48 Monaten fällt die Kurve ein weiteres Mal auf 96,23\%. Der dritte Verlust trat nach 20,69 Monaten auf, wobei die Kurve auf 94,22\% abfällt. Nach ca. 48 Monaten kam es zum vierten Verlust, sodass die Kurve nochmals um ca. 4\% auf 90,13\% absinkt (s. Tab.7 und Abb.13). 
Tab.7: Überlebensanalyse bezogen auf die Kieferregionen, Überlebenszeit: T(m), Überlebenswahrscheinlichkeit: \%

\begin{tabular}{|c|c|c|}
\hline \multirow{2}{*}{ Überlebenszeit } & \multicolumn{2}{|c|}{ Überlebens- } \\
& wahrscheinlichkeit \% \\
\cline { 2 - 3 } & Unterkiefer & Oberkiefer \\
\hline 13,31 & & 98,11 \\
\hline 15,48 & & 96,23 \\
\hline 19,51 & 96,78 & \\
\hline 20,69 & & 94,22 \\
\hline 47,93 & & 90,13 \\
\hline
\end{tabular}

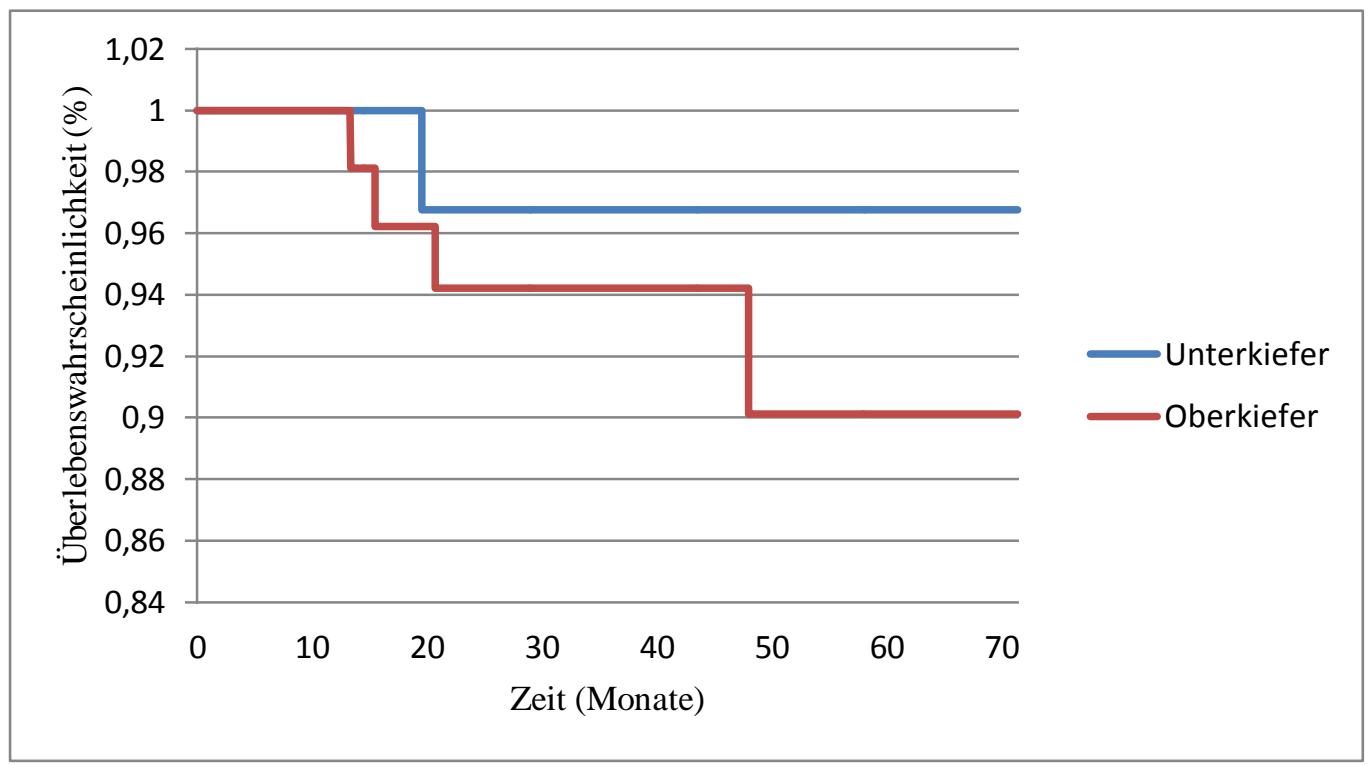

Abb.13: Überlebensanalyse bezogen auf die Kieferregionen in Abhängigkeit von der Zeit

\subsubsection{Schätzung der Überlebensrate}

Die Schätzung der Überlebensrate sowie die weiteren Schätzungen Kaplan-MeierAnalyse sind univariat. Hierbei werden die Konfidenzintervalle betrachtet.

Das Konfidenzintervall beschreibt die Präzision der Lageschätzung eines Parameters. Das Vertrauensintervall schließt einen Bereich um den geschätzten Wert des Parameters ein, der mit einer zuvor festgelegten Wahrscheinlichkeit die wahre Lage des Parameters trifft. 
Den Werten der Tabelle 8 kann man entnehmen, dass sowohl die Schätzungen als auch die Konfidenzintervallgrenzen im Bezug auf Zement und Region in einem ähnlichen Bereich liegen.

Nach 36 Monaten Beobachtungszeit liegen die Werte der Schätzung z. B. für Variolink bei 93,88\% und für Calibra bei 96,88\%. Gleiches gilt für die Regionen. Hier liegt die Schätzung für den Unterkiefer bei 96,77\% und für den Oberkiefer bei 94,22\%. Ähnlich ist es auch bei den Konfidenzintervallgrenzen. Hier unterscheiden sich vor allem die Zemente deutlicher. Für Calibra liegen die Grenzen zwischen 0,92 und 1 und für Variolink zwischen 0,85 und 1. Die Grenzen für die Regionen liegen in einem ähnlichen Bereich von etwa 0,87 und 1. Der Overall-Verlust liegt in einem Grenzintervall von 0,88 und 1 bei 95,12\%.

Tab.8: Schätzung der Überlebenswahrscheinlichkeit in Prozent (\%)

\begin{tabular}{|c|c|c|}
\hline & Schätzung \% & $\begin{array}{c}95 \% \text {-Konfidenz- } \\
\text { intervallgrenzen }\end{array}$ \\
\hline Zement (Calibra) & 96,88 & $0.92-1$ \\
\hline Zement(Variolink) & 93,88 & $0.85-1$ \\
\hline Region (Unterkiefer) & 96,77 & $0.88-1$ \\
\hline Region (Oberkiefer) & 94,22 & $0.87-1$ \\
\hline Overall-Verlust & 95,12 & $0,88-1$ \\
\hline
\end{tabular}

\subsubsection{Erfolgsrate}

Die Erfolgsrate beschreibt die relative Häufigkeit, mit der ein bestimmtes Ereignis, wie z.B. das Rezementieren oder eine Wurzelkanalbehandlung, aber auch die Anzahl an Verlusten der Restaurationen, bei einer Gruppe von Patienten beobachtet wird. In der Graphik (s. Abb.15) sind alle Ereignisse, d.h. Rezementierungen, Extraktionen, Verluste und Wurzelkanalbehandlungen (Endo) dargestellt.

Im Beobachtungszeitraum von 36 Monaten wurde einmal nach 1,77 und zweimal nach 61,08 Monaten ein Veneer rezementiert. In diesen Fällen wurde Variolink als Zement benutzt. Eine endodontische Behandlung (s. Abb. 14) war nach 2,07 Monaten nötig. 


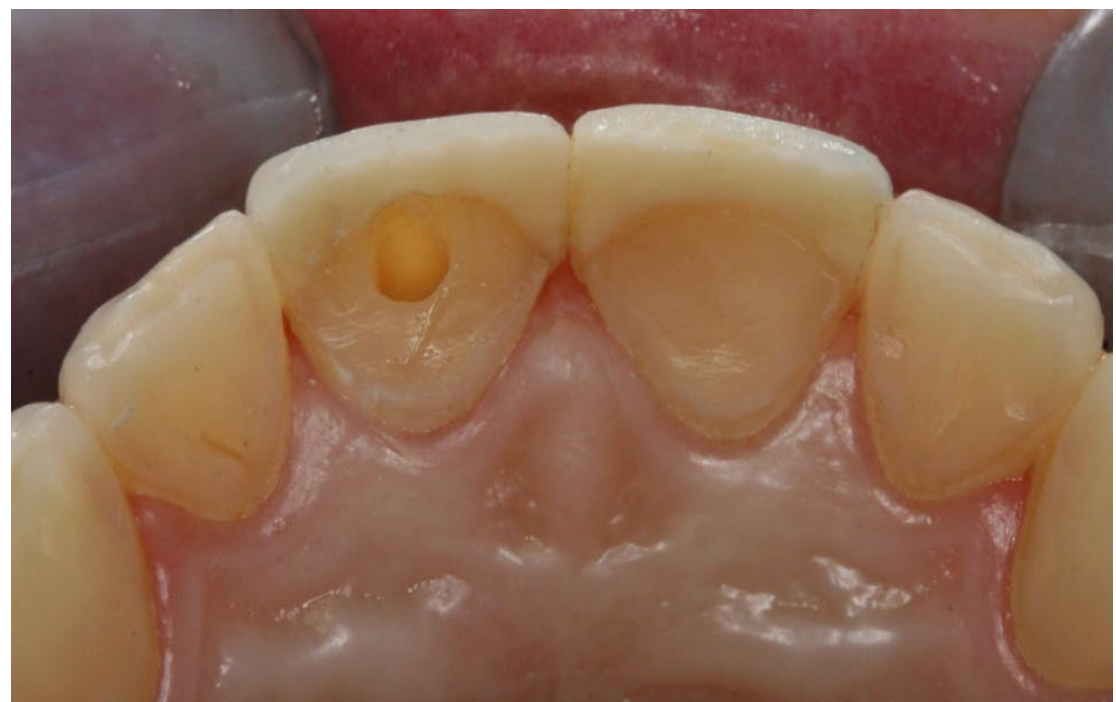

Abb. 14.: endodontische Behandlung bei einem mit einem Veneer versorgten Zahn (11)

Diese Ereignisse erklären den Abfall der Erfolgsrate zunächst von 100\% auf 99,23\%, anschließend auf 98,46\%. . Ein weiteres Ereignis trat nach 7,84 Monaten auf. Hier musste eine Wurzelkanalbehandlung eines mit einem Veneer versorgten Zahns durchgeführt werden. Dies führt zu einem Abfall der Kurve auf 97,6\%. Hierauf folgen die bereits beschriebenen fünf Verluste nach 13,31, 15,48, 19,51, 20,69 und 47,93 Monaten. In diesem Zeitraum sinkt die Erfolgswahrscheinlichkeit von 96,5\% auf 88,74\%. Nach 61,08 Monaten kommt es zum stärksten Einbruch um knapp 20\% auf 69,02\% aufgrund von zwei weiteren Rezementierungen (s. Tab.9).

Tab.9: Erfolgsanalyse, Erfolgszeit T(m), Erfolgswahrscheinlichkeit: \%, Art der Ereignisse

\begin{tabular}{|c|c|c|}
\hline \multirow{2}{*}{ Erfolgszeit } & $\begin{array}{c}\text { Erfolgs- } \\
\text { wahrscheinlichkeit \% }\end{array}$ & Art der Ereignisse \\
\hline 1,77 & 99,23 & Rezementierung \\
\hline 2,07 & 98,46 & Endo \\
\hline 7,84 & 97,6 & Endo \\
\hline 13,31 & 96,5 & Fraktur \\
\hline 15,48 & 95,32 & Extraktion \\
\hline 19,51 & 94,1 & Fraktur \\
\hline 20,69 & 92,78 & Fraktur \\
\hline 47,93 & 88,74 & Fraktur \\
\hline 61,08 & 69,02 & Rezementierungen \\
\hline
\end{tabular}




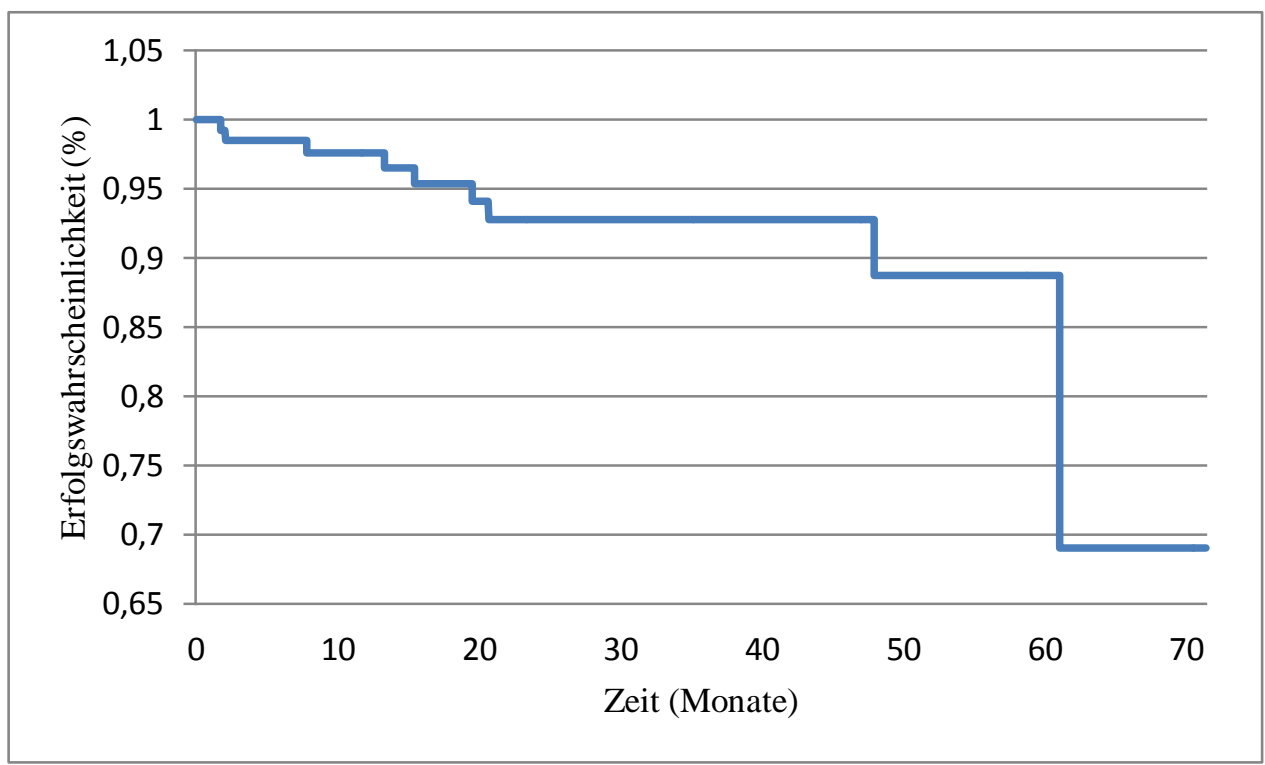

Abb.15: Erfolgsanalyse in Abhängigkeit von der Zeit

\subsubsection{Faktor Zement}

Das höher geschätzte Risiko bei den mit Variolink befestigten Veneers, vgl. Kapitel 4.3.1.1, spiegelt sich auch beim Betrachten der Erfolgsrate nach 36 Monaten wider. Das Risiko, dass bei einem mit Variolink befestigten Veneer ein Ereignis eintritt, liegt nach 36 Monaten bei 1,18. Der p-Wert von 0,78 deutet jedoch wiederum darauf hin, dass keine Signifikanz vorliegt.

In Abbildung 16 wird deutlich, dass sich die Kurven der beiden Zemente in den ersten vier Jahren in einem ähnlichen Bereich bewegen. Erst ab dem Monat 47,93 beginnt eine deutlichere Differenzierung zwischen den mit Variolink und den mit Calibra befestigten Veneers.

Die Anzahl der mit Calibra zementierten Versorgungen bleibt nach drei kleinen Einbrüchen stabil. Nach 2,07 und nach 7,84 Monaten wurde jeweils eine Wurzelkanalbehandlung bei einem mit einem Veneer versorgten Zahn notwendig. Daraus ergibt sich, dass die Erfolgswahrscheinlichkeit zunächst nach 2,07 Monaten auf 98,59\%, dann nach 7,84 Monaten auf 96,86\% und schließlich nach 19,51 Monaten aufgrund eines Verlusts auf 93,83\% sinkt.

Bei den mit Variolink befestigten Veneers kommt es neben den aufgetretenen Verlusten nach 36 Monaten zu drei weiteren Ereignissen, eins nach 1,77 Monaten und zwei nach 61,08 Monaten. Nach 1,77 Monaten sinkt die Erfolgswahrscheinlichkeit aufgrund der Notwendigkeit einer Rezementierung von 100\% auf 98,31\%. Darauf folgen vier Verluste wie sie in Kapitel 4.3.1.1 erwähnt sind. Somit kommt es nach 
13,31 Monaten zu einem Abfall der Kurve auf 96,26\%, nach 15,48 Monaten auf 94,21\%. Nach 20,69 Monaten sinkt sie von 94,21\% auf 92,16\%, nach 47,93 Monaten auf 87,97\% und schließlich nach 61,08 Monaten um knapp 20\% auf 68,42\% (s. Tab.10). Dieser starke Abfall wird von den beiden nach 61,08 Monaten aufgetretenen Rezementierungen ausgelöst.

Tab.10: Erfolgsanalyse bezogen auf die Befestigungszemente, Erfolgsszeit: T(m), Erfolgswahrscheinlichkeit: \%

\begin{tabular}{|c|c|c|}
\hline \multirow{2}{*}{ Erfolgszeit } & \multicolumn{2}{|c|}{ Erfolgs- } \\
\cline { 2 - 3 } & Cahrscheinlichkeit \% \\
\hline 1,77 & & Variolink \\
\hline 2,07 & 98,59 & \\
\hline 7,84 & 96,86 & \\
\hline 13,31 & & 96,26 \\
\hline 15,48 & & 94,21 \\
\hline 19,51 & 93,83 & \\
\hline 20,69 & & 92,16 \\
\hline 47,93 & & 87,97 \\
\hline 61,08 & & 68,42 \\
\hline
\end{tabular}

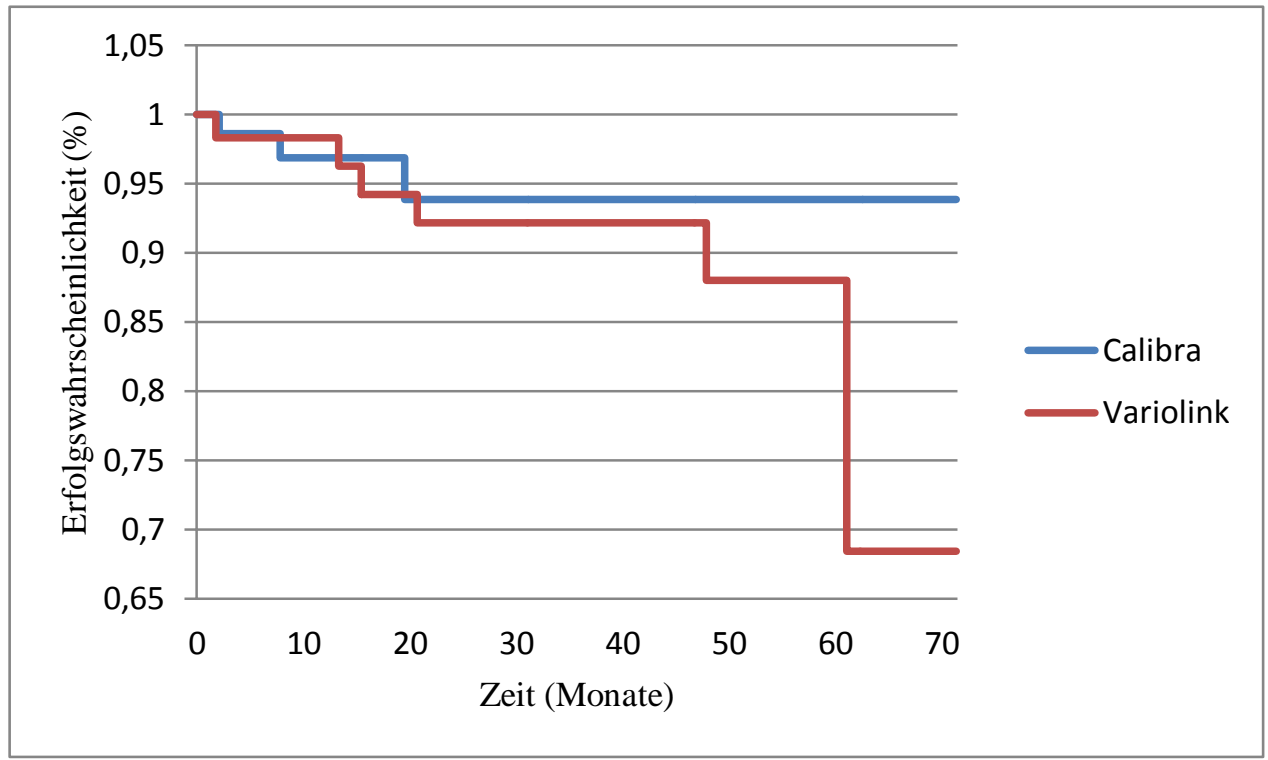

Abb.16: Erfolgsanalyse bezogen auf die Befestigungszemente in Abhängigkeit von der Zeit 


\subsubsection{Faktor Region}

Im Bezug auf die Erfolgsrate liegt das geschätzte Risiko nach einem Beobachtungszeitraum von 36 Monaten für den Oberkiefer um den Faktor 1,64 (Kehrwert von 0,609) höher als für den Unterkiefer. Jedoch besteht keine Signifikanz, da der pWert bei 0,4 liegt.

Der Unterkiefer weist zwei Ereignisse auf. Nach 1,77 Monaten kommt es zu der bereits beschriebenen Rezementierung, die für einen Abfall von 100\% auf 98,15\% verantwortlich ist. Nach 19,51 Monaten folgt der Verlust eines weiteren Veneers. Es sinkt die Anzahl der intakten Veneers von 98,15\% auf 94,88\%.

Im Oberkiefer sind acht Ereignisse zu nennen - je eins nach 2,07, 7,84, 13,31, 15,48, 20,69, 47,93 und zwei nach 61,08 Monaten. Es kommt zu den Abfällen von 100\% auf 98,68\%, dann auf 97,07\%, anschließend auf 95,23\%, danach auf 93,4\%, dann auf 91,46\%, hierauf folgend auf $87,48 \%$ und letztendlich auf $68,04 \%$.

Auch hier verläuft die Kurve (s. Abb.17), die die Erfolgswahrscheinlichkeit der Veneers im Oberkiefer darstellt, bis zum etwa 48. Monat in einem ähnlichen Bereich wie die der Unterkieferveneers. Erst nach knapp vier Jahren kommt es zum stärkeren Abfall der Werte für den Oberkiefer bis auf 68,04\% nach 61,08 Monaten (s. Tab.11).

Tab.11: Erfolgsanalyse bezogen auf die Kieferregionen, Erfolgszeit: T(m),

Erfolgswahrscheinlichkeit: \%

\begin{tabular}{|c|c|c|}
\hline \multirow{2}{*}{ Erfolgszeit } & \multicolumn{2}{|c|}{$\begin{array}{c}\text { Erfolgs- } \\
\text { wahrscheinlichkeit \% }\end{array}$} \\
\cline { 2 - 3 } & Unterkiefer & Oberkiefer \\
\hline 1,77 & 98,15 & \\
\hline 2,07 & & 98,68 \\
\hline 7,84 & & 97,07 \\
\hline 13,31 & & 95,23 \\
\hline 15,48 & & 93,4 \\
\hline 19,51 & 94,88 & \\
\hline 20,69 & & 91,46 \\
\hline 47,93 & & 87,48 \\
\hline 61,08 & & 68,04 \\
\hline
\end{tabular}




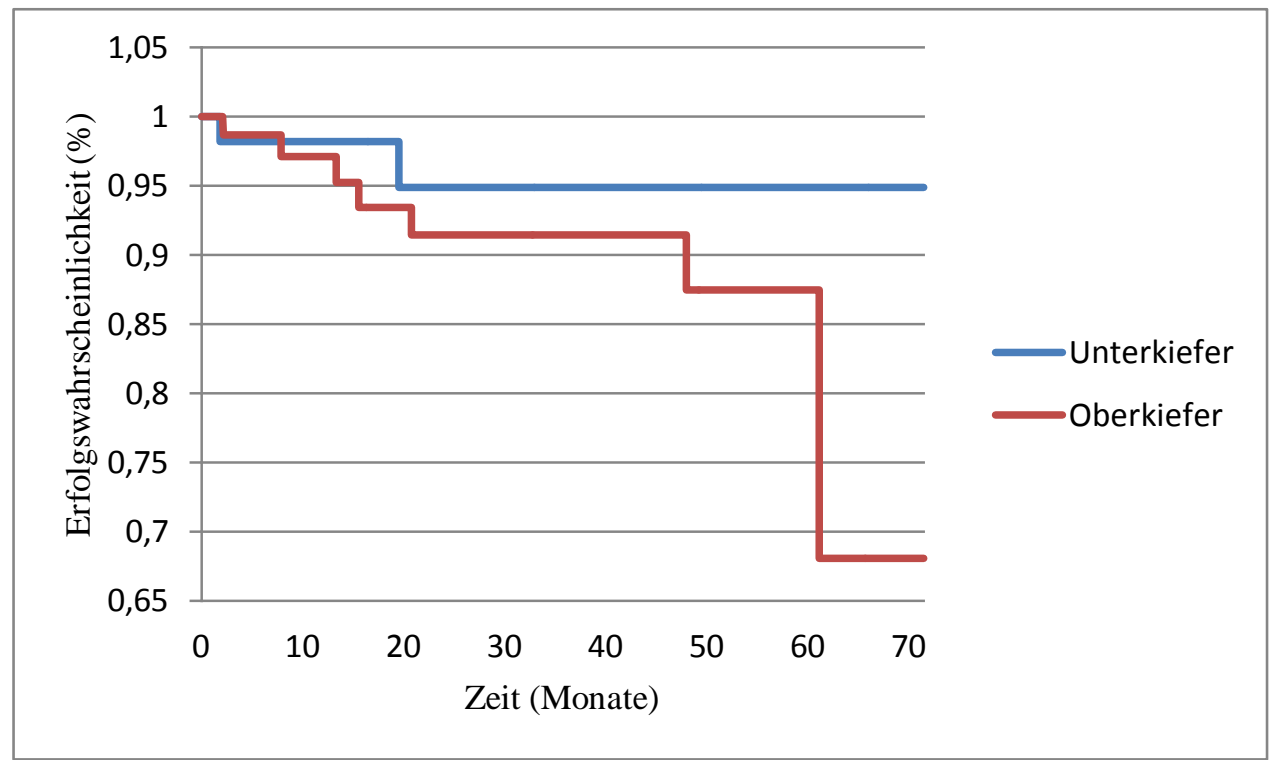

Abb.17: Erfolgsanalyse bezogen auf die Kieferregionen in Abhängigkeit von der Zeit

\subsubsection{Schätzung der Erfolgsrate}

Die Schätzung der Erfolgsrate ist ebenfalls univariat - entsprechend der KaplanMeier-Analyse.

Die Werte hinsichtlich der Schätzungen weisen keine großen Differenzen auf, weder im Bezug auf den Zement noch hinsichtlich der Region. Diese liegen nach 36 Monaten sowohl für die beiden Zemente als auch für die beiden Regionen in einem ähnlichen Bereich von 91-95\%. Anders stellt es sich bei den Konfidenzintervallgrenzen dar. Hier variieren die Grenzen zwischen 0,87-1 (Calibra) und 0,62-1 (Unterkiefer). Der Overall-Verlust liegt bei 92,78\% mit den Intervallgrenzen von 0,86 bis 1 (s. Tab.12).

Tab.12: Schätzung der Erfolgswahrscheinlichkeit in Prozent (\%)

\begin{tabular}{|c|c|c|}
\hline & Schätzung \% & $\begin{array}{c}95 \%- \\
\text { Konfidenzintervallgrenzen }\end{array}$ \\
\hline Zement (Calibra) & 93,83 & $0.87-1$ \\
\hline Zement(Variolink) & 92,16 & $0.82-1$ \\
\hline Region (Unterkiefer) & 94,88 & $0.62-1$ \\
\hline Region (Oberkiefer) & 91,46 & $0.83-1$ \\
\hline Overall-Verlust & 92,78 & $0,86-1$ \\
\hline
\end{tabular}




\subsubsection{Endergebnisse}

Die durchschnittliche Überlebensrate lag nach 36 Monaten bei ca. 97,3\%, die Erfolgsrate nach drei Jahren bei ca. 95\%. Insgesamt kam es zu zehn Ereignissen, wobei es sich bei fünf dieser Ereignisse um Verluste, im Sinne von Frakturen oder biologischem Misserfolg handelte, und bei fünf weiteren um Rezementierungen oder Wurzelkanalbehandlungen (s. Tab. 13). Demnach kam es bei 3,85\% der Patienten zu einem Verlust und bei 7,69\% der Patienten zu einem der aufgeführten Ereignisse.

Tab. 13: Gründe für Verluste und Ereignisse

\begin{tabular}{|c|c|c|}
\hline Anzahl & $\begin{array}{c}\text { Verhältnis zu } \\
\text { Gesamtzahl der } \\
\text { untersuchten Zähne }\end{array}$ \\
\hline Verluste insgesamt & 5 & $3,85 \%$ \\
\hline Keramikfrakturen & 4 & $3,08 \%$ \\
\hline Extraktionen & 1 & $0,77 \%$ \\
\hline nötige Interventionen & 5 & $3,85 \%$ \\
\hline Rezementierungen & 3 & $2,31 \%$ \\
\hline $\begin{array}{c}\text { endodontische } \\
\text { Behandlung }\end{array}$ & 2 & $1,54 \%$ \\
\hline Ereignisse insgesamt & 10 & $7,69 \%$ \\
\hline
\end{tabular}

Statistisch gesehen ist der Unterschied zwischen den Versorgungen in beiden Kiefern nicht signifikant, weder für die Überlebensrate $(p=0,3)$ (s. Tab. 14) noch für die Erfolgsrate $(p=0,4)$ (s. Tab. 15). Ähnliches gilt auch für die Wahl des Befestigungszements. Hier ist p für die Überlebensrate 0,2 und für die Erfolgsrate 0,78.

Tab. 14: Hazard-Ratio und Signifikanz der Zemente und Regionen im Bezug auf die Verlustrate

\begin{tabular}{|c|c|c|}
\hline Zement & Hazard-Ratio & $\begin{array}{c}\text { Signifikanz } \\
\text { (p-Wert) }\end{array}$ \\
\cline { 1 - 2 } Variolink & 2,13 & \multirow{2}{*}{0,2} \\
\cline { 1 - 2 } Calibra & 0,47 & Signifikanz \\
Region & Hazard-Ratio & \multirow{2}{*}{0,3} \\
\hline Oberkiefer & 1,82 & \\
\hline Unterkiefer & 0,55 & \\
\hline
\end{tabular}


Tab. 15: Hazard-Ratio und Signifikanz der Zemente und Regionen im Bezug auf die Erfolgsrate

\begin{tabular}{|c|c|c|}
\hline Zement & Hazard-Ratio & $\begin{array}{c}\text { Signifikanz } \\
\text { (p-Wert) }\end{array}$ \\
\hline Variolink & 1,18 & \multirow{2}{*}{0,78} \\
\hline Calibra & 0,85 & Signifikanz \\
(p-Wert)
\end{tabular}




\section{Diskussion}

\subsection{Material}

\subsubsection{Probandenauswahl}

Die Probanden dieser Studie waren allesamt Patienten der Zahnarztpraxis Dr. med. dent. S. Rinke \& S. Schmidt. Hierdurch entfielen praxisspezifische Besonderheiten als Einflussgrößen auf die Untersuchungsergebnisse. Oft ist es ein Problem, die Datenerfassung so zu gestalten, dass vergleichbare Ergebnisse herauskommen. Dies ist hier kein mit einfließender Faktor, da alle Veneers mit einem standardisierten Verfahren eingesetzt und in regelmäßige Abständen befundet wurden. Daraus lässt sich schließen, dass die vorliegenden Daten repräsentativ und miteinander vergleichbar sind.

Ein limitierender Faktor retrospektiver Studien ist die zuverlässige Teilnahme der Patienten/Probanden an der gesamten Studie, d.h. regelmäßige Wahrnehmung der Kontrolltermine. Somit können die aussagekräftigen Daten nur in Abhängigkeit von der Konstanz des Patientenstamms erfasst werden. Von ursprünglich 41 Patienten hatten sich 37 der Behandelten dazu bereit erklärt, an der Langzeituntersuchung teilzunehmen. Dementsprechend lag die Rate bei 90,2\%. Hierdurch konnte die Unzuverlässigkeit und somit die Unzufriedenheit der Patienten, an der Studie teilzunehmen, minimiert werden.

Voraussetzung für die Einbeziehung der Patienten in die Untersuchung waren die Anforderungen, dass das gesamte Gebiss kariesfrei und suffizient versorgt war. Die Zähne, die mit einem Veneer zu restaurieren waren, durften lediglich zwei Kompositfüllungen aufweisen. Als Indikation für die Versorgung mit einem Keramikveneer kamen Diastema, Verfärbungen, Hypoplasie und Zahnfehlstellung in Frage.

Für die Vergleichbarkeit der Ergebnisse war es neben der Kariesfreiheit wichtig, nur Patienten in die Studie einzubeziehen, die ein oder mehrere Veneers in der Oberoder Unterkieferfront aufwiesen, deren behandelte Zähne vital und funktionsdiagnostisch unauffällig waren. Lagen bei Patienten Funktionsstörungen, Infektionskrankheiten, große keilförmige Defekte etc. (s. Kapitel 3.2.1.2) vor, so wurden diese aus der Studie ausgeschlossen. 
Die hohen Anforderungen an die Probanden führten zu einem Missverhältnis zwischen männlichen und weiblichen Patienten, einer ungleichen Altersverteilung der Patienten und den bereits angesprochenen geringen Stichprobenumfängen.

\subsubsection{Bewertung der Präparation, des Adhäsivsystems und der Zemente}

Alle 130 in die Untersuchung einbezogenen Veneerpräpartionen wurden von ein und demselben Zahnarzt, Dr. med. dent. Rinke, durchgeführt. Aufgrund seiner jahrelangen Erfahrung ist von einer Konstanz in der Präparationstechnik und -sorgfalt auszugehen. Als Präparationsset wurde das ERGO 4395 von Komet benutzt. Da auch der Ort der Behandlung immer der gleiche war, sind Fehler wie ungenügende Wasserkühlung bei unzureichender Wasserzufuhr oder verstopfte Düsen am Winkelstück, abgenutzte Diamanten, abweichende Rotationsgeschwindigkeiten der Diamanten etc., weitgehend auszuschließen. Somit kann davon ausgegangen werden, dass ein Vitalitätsverlust durch unsachgemäße Präparation und Überhitzung nicht eintrat.

Bei allen in dieser Studie festgestellten Frakturen wurde zuvor bis in das Dentin hinein präpariert. Diese Dentinexposition ist bei insgesamt kleinen Zähnen, vor allem in der Unterkieferfront oftmals nicht zu vermeiden. Somit scheint die Tiefe der Präparation eine Rolle hinsichtlich des Frakturrisikos zu spielen

Als Design wurde in allen Fällen eine Overlap-Präparation gewählt. Diese Form zeichnet sich durch gute ästhetische Ergebnisse, aber auch durch die Möglichkeit einer okklusalen Korrektur aus (DUMFAHRT und SCHAFFER 2000).

Bei allen untersuchten Veneers dieser Studie wurde Optibond FL als Adhäsivsystem verwendet, so dass deren Überlebens- und Erfolgsraten diesbezüglich nicht beeinflusst waren.

Keramik weist gegenüber dem Zahn eine verringerte Biegefestigkeit auf. Dies macht einen vollständigen Klebeverbund zwischen Restauration und Zahnhartsubstanz erforderlich, um die Abscherkräfte abzufangen und die Langlebigkeit des Veneers zu garantieren.

In dieser Studie wurden, wie in der Literatur empfohlen, die Veneeroberflächen geätzt und silanisiert (SIMONSEN und CALAMIA 1983, CALAMIA 1985, FRIEDMAN 1998, HAHN et al. 2000). Die Zemente ermöglichen aufgrund ihrer hohen Anteile an Füllkörpern eine hohe Festigkeit bei geringer Polymerisationsschrumpfung und zudem eine geringe thermische Expansion (SIM et al. 1994). Dies führt 
dazu, dass der Randspalt kleiner und somit die Gefahr einer Sekundärkaries minimiert wird (PEUMANS et al. 2000).

In der Studie kamen zwei verschiedene Systeme an Befestigungszementen zum Einsatz. Die Verteilung der Zemente auf die Patienten war willkürlich und folgte dem Zufallsprinzip. 59 (45\%) der Veneers wurden mit Variolink (Ivoclar Vivadent, Schaan/Liechtenstein) und 71 (55\%) Veneers mit Calibra (Dentsply, Konstanz/Deutschland) befestigt.

Beide Zemente entsprechen den hohen ästhetischen Anforderungen der Restauration mit einem Keramikveneer. Da das Veneer zum Einen sehr dünn und zum Anderen fast transparent ist, ist Transparenz des Zements Voraussetzung für den objektiven, aber auch für den subjektiven, d.h. vom Patienten empfundenen Erfolg. Dieser sogenannte Chamäleoneffekt ermöglicht somit die Farbangleichung der Restauration an die Nachbarzähne.

Klinisch haben sich beide Zemente sowohl in der Anwendung als auch in der Verträglichkeit bewährt, wobei laut WANG et al. (2007) für Variolink eine geringere Haftfestigkeit vorliegt als für Calibra.

\subsubsection{Bewertung der verwendeten Presskeramik}

In dieser Studie wurden alle 130 untersuchten Veneers aus Presskeramik (Cergo) hergestellt. Cergo (DeguDent GmbH, Hanau/Deutschland) zeichnet sich dadurch aus, dass es sehr gut für ästhetische Zwecke, wie hier Veneers, geeignet ist. Doch auch die mechanischen Eigenschaften sind als gut zu bezeichnen. So liegt z.B. laut Herstellerangaben eine naturnahe Oberfläche vor, was dazu führt, dass das Abrasionsverhalten dem eines natürlichen Zahnes ähnelt. Bei Okklusion wird somit weder bei der Restauration, noch bei der Zahnhartsubstanz eine übermäßige Abrasion provoziert.

Allgemein hat sich die Presskeramik durch ihre gute Biokompatibilität und ihre mechanischen Eigenschaften klinisch bewährt (YILMAZ et al. 2007).

Da alle in dieser Studie untersuchten Veneers aus Cergo-Presskeramik bestehen, ist neben den herstellungsbedingten Fehlerquellen kein Einfluss auf die Überlebens- und Erfolgsrate abzuleiten. 


\subsection{Methodik}

\subsubsection{Problematik von in-vivo-Studien}

In-vivo-Studien unterscheiden sich von in-vitro-Studien dadurch, dass die Untersuchungen am lebenden Objekt stattfinden. Dies hat bestimmte Vor- aber auch Nachteile. Die Problematik liegt hierbei zum Einen darin, dass, aufgrund der individuellen Mundverhältnisse bei jedem Patienten, keine gleichmäßig über die gesamte Probandenanzahl verteilten mechanischen Belastungen vorliegen. DELLA BONA und KELLY (2010) haben hierzu bestimmte Faktoren beschrieben, die die Überlebensbzw. Erfolgswahrscheinlichkeit beeinflussen. So wurde festgestellt, dass es im Vergleich der Geschlechter einen Unterschied gibt, denn hier ist die Überlebenszeit der Veneers bei männlichen Patienten deutlich geringer, als die bei weiblichen Personen $(\mathrm{p}=0,01)$. Auch das Alter scheint eine Rolle zu spielen, denn ab dem Alter von 60 Jahren nimmt die Überlebenszeit der Restauration signifikant ab $(\mathrm{p}=0,003)$. Diese Beobachtungen lassen sich in dieser Studie nicht bestätigen, da die Verteilung in den einzelnen Altersgruppen zu ungleichmäßig war, um aussagekräftige Ergebnisse zu formulieren. Eine weitere Einflussgröße ergibt sich aus dem Umstand, wie oft der Patient den behandelnden Zahnarzt gewechselt hat, denn je öfter dies vorkommt, desto häufiger kommt es zum Verlust eines Veneers $(\mathrm{p}=0,001)$. Dies lässt sich in dieser Studie ausschließen, weil alle Veneers von ein und demselben Behandler eingesetzt und, bis auf die für diese Studie notwendigen Untersuchungstermine, kontrolliert wurden.

Ein weiterer, für die Auswertungen wichtiger Punkt, ist die Verteilung der Veneers auf die Zähne des Ober- bzw. Unterkiefers. Bei in-vitro-Studien hat man die Möglichkeit, vor Studienbeginn die Verteilung zu beeinflussen, indem man gleichmäßig viele Zähne für jeden Kiefer sammelt und anschließend untersucht. Dies ist bei invivo-Studien nicht möglich, da sich die Verteilung der Veneers nach der jeweiligen Indikation richtet. In dieser Untersuchungsreihe ist es jedoch gelungen, einigermaßen gleichmäßig viele Veneers im Ober- $(n=76)$ bzw. Unterkiefer $(n=54)$ einzubeziehen. Des Weiteren ist die Zuverlässigkeit des Patienten ein wichtiger Einflussfaktor. Um nach Abschluss der Untersuchungen aussagekräftige Ergebnisse zu erzielen, ist es wichtig, dass über die gesamte Studie hinweg die Patienten in zueinander gleichmässigen Abständen untersucht werden. Dies war hier gegeben. Indem vor Beginn eine 
Einverständniserklärung vom Patienten unterschrieben wurde, konnte man die Patienten, die nicht an der Studie über einen längeren Zeitraum teilnehmen wollten, aussortieren und somit das Risiko eines Nichterscheinens zum abgesprochenen Termin minimieren.

Außerdem war es wichtig, die Patientengewohnheiten in der Anamnese und Diagnostik so gut wie möglich zu analysieren, um Risikofaktoren wie Pressen und Knirschen zu erkennen und den Patienten aus der Studie auszuschließen.

\subsubsection{Beurteilung der Untersuchungsmethodik}

Die nach 36 Monaten durchgeführten Untersuchungen wurden alle von ein und demselben Zahnarzt durchgeführt.

Nachdem die Zähne und die Veneers einer zahnärztlichen Standarduntersuchung mit Sonde, Spiegel und Zahnseide unterzogen worden waren, wurden intraorale Fotoaufnahmen angefertigt. Des Weiteren wurden die Restaurationen hinsichtlich Randdichtigkeit, Randverfärbung, Sekundärkaries etc. beurteilt und der Erfolg bzw. Misserfolg bestimmt.

In dieser Studie war es lediglich eine Ja- oder Nein-Entscheidung, ob der jeweilige Fall aufgetreten war oder nicht. Der ursprüngliche USPHS unterscheidet in jeder Kategorie verschiedene Ausprägungen der Fehler (s. Anhang Tab. 16). Diese Tatsachenentscheidung hat die Schwäche einer absoluten Entscheidung zwischen Erfolg und Misserfolg. So ist auch eine kleine Komplikation, die leicht behoben werden könnte, als Misserfolg eingestuft worden. Kleine Abstufungen ermöglichen hingegen eine feiner abgestimmte Entscheidung zu treffen, um so die Rate an Misserfolgen zu minimieren.

Außerdem ist der Druck der Sonde, als taktiles Verfahren bei Verwendung einer Standard-Sonde, nicht immer mit der gleichen Kraft anzuwenden. Diese Abweichungen führen dann zu Ungenauigkeiten bei der Bestimmung der objektiven Kriterien wie Randspalt oder Sekundärkaries.

Zur Beurteilung der Randschlussqualität könnte man Abformungen und daraus wiederum Modelle anfertigen, die sich unter einem Raster-Elektronen-Mikroskop beurteilen lassen. Dies war zunächst eine Überlegung in der Planung dieser Studie, jedoch fehlten hierzu Ursprungsbilder, die einen Vergleich in Abhängigkeit von der Zeit erlaubt hätten. 
Die Bewertung der farblichen Anpassung, der anatomischen Veneerform sowie der Verfärbung sind dagegen subjektiv, was in der Studie von MEIJERING et al. (1997) belegt wurde. Hier wird gezeigt, dass das, was der Zahnarzt als ästhetisch empfindet, nicht immer auch beim Patienten zu Zufriedenheit führt und umgekehrt. Die Zufriedenheit nimmt mit zunehmender Zeit ab, da sich der Patient an den neuen Zustand gewöhnt hat und somit die ästhetischen Ansprüche gestiegen sind. Dieser Unterschied ist bei Keramikveneers am geringsten.

Des Weitern stellt sich die Frage, ob die Aussagen über die Überlebens- und Erfolgsraten aussagekräftiger wären, wenn mehrere Zahnärzte die vorhandenen Veneers mit der selben Methode untersucht hätten, um so die subjektiven Faktoren in Ihrem Einfluss zu minimieren.

Ein weiterer Faktor, der die Aussagekraft der in dieser Studie ermittelten Daten minimiert, ist die kurze Reevaluationszeit von drei Jahren. Hier ist sicherlich eine weitere Untersuchung nach fünf, zehn bzw. 15 Jahren wie in anderen Studien notwendig, um die Ergebnisse zu bekräftigen. Danach wird der Einfluss der Position des Veneers bzw. des Befestigungszements auf die Überlebens- bzw. Erfolgsrate voraussichtlich noch deutlicher.

\subsection{Ergebnisse}

In der vorliegenden Studie lag die durchschnittliche Überlebensrate nach 36 Monaten bei ca. 97,3\%. Dieses Ergebnis unterscheidet sich um etwa 5\% von dem von KREULEN et al. (1998) beschriebenen Wert von 92\% nach drei Jahren Beobachtungszeit. Bei Betrachtung der Überlebenswahrscheinlichkeiten aller Keramiksysteme liegen die Fehlerquoten zwischen 5\% und 10\%. Nur für gepresste Keramik-Veneers wurde bisher eine Überlebenswahrscheinlichkeit von 96,5-98,8\% nach viereinhalb bis sechs Jahren Beobachtung festgestellt (FRADEANI 1998, ARISTIDIS und DIMITRA 2002, GUESS und STAPPERT 2008).

Die Fraktur der Keramik war in vier von fünf Fällen der Grund für einen Verlust und stellte somit die Hauptursache dar. Insgesamt lag die Frakturrate bei 3\%. In anderen Studien wurden Raten zwischen 0-3\% festgestellt, wobei ebenfalls die Verluste auf Frakturen zurückzuführen waren und eine Behandlung notwendig machten (MAGNE und DOUGLAS 2000, PEUMANS et al. 2004, DUMFAHRT und SCHAFFER 2000, 
GUESS und STAPPERT 2008, MURPHY et al. 2005, FRIEDMAN 1998, LAYTON und WALTON 2007).

Auffallend an dieser Studie ist die Beobachtung, dass drei der vier Frakturen bei ein und demselben Patienten auftraten. Auf Befragung an den Kontrollterminen erklärte dieser Patient, dass er nach Einsetzen der Veneers privat unter starkem Stress litt (Scheidung und finanzielle Probleme) und bei sich selbst parafunktionelle Aktivitäten, wie Knirschen und Pressen, beobachtete. Diese Aspekte müssen bei der Interpretation der Ergebnisse beachtet werden. Sie bestärken die verbreitete Ansicht, dass patientenbezogene Faktoren wie durch Stress ausgelöste Parafunktionen einen großen Einfluss auf die Fehlerquote und somit die Überlebenswahrscheinlichkeit von Keramikrestaurationen haben. Bestätigt wird diese Auffassung durch mehrere Studien, in denen die Überlebensrate der Veneers im Zusammenhang mit stressprovoziertem Knirschen und Pressen stand und sich bei diesen Patienten das Risiko erhöhte, dass die Veneers frakturierten (DUMFAHRT und SCHAFFER 2000, FRIEDMAN 1998).

Die Erfolgsrate lag in der vorliegenden Untersuchung nach drei Jahren bei ca. 95\%. Im Vergleich zu anderen Arbeiten ist dieser Wert im besseren Bereich angesiedelt. Dies unterstreicht z.B. ein Vergleich mit der von GUESS und STAPPERT (2008) durchgeführten Studie, bei der ebenfalls eine gepresste Keramik (IPS Empress) verwandt wurde und die Erfolgsraten nach fünf Jahren zwischen 72\% und 85\% lagen. Die Präparationen der einzelnen Zähne waren jedoch bei ihnen verschieden.

Um die Funktion des Veneers und somit des Zahns zu erhalten, musste in dieser Untersuchungsreihe in fünf Fällen eingegriffen werden. Zweimal waren eine Wurzelkanalbehandlung und dreimal eine Rezementierung nötig. Dementsprechend lag die Rate der sich ablösenden Veneers bei 2,3\%. Es konnten jedoch alle Veneers durch eine Rezementierung in ihrer Funktion erhalten werden. In allen Eingriffsfällen, bei denen sich das Veneer von der Zahnoberfläche gelöst hatte, ragten die Präparationen bis tief in das Dentin hinein. Dieser Zusammenhang wurde auch schon in früheren Studien beschrieben, in denen die Rezementierungsrate bis zu 11\% erreichte (MURPHY et al. 2005, DUNNE und MILLAR 1993, FRIEDMAN 1998). Hauptursachen waren entweder ein unpräparierter Schmelz oder das Kleben der Veneers auf große Kompositfüllungen. Nach Untersuchungen von SHAINI et al. (1997) wird zudem das Risiko erhöht, wenn vor der Zementierung die Restauration nicht mit Fluoressigsäure behandelt wird. 
Die in dieser Studie verhältnismäßig geringe Rate an abgelösten Veneers ist vor allem darauf zurückzuführen, dass alle Zähne präpariert, alle Veneers mit Flusssäure behandelt und alle Restaurationsränder auf gesunder Zahnhartsubstanz zementiert wurden. Nicht unerwähnt bleiben soll aber, dass in dieser Studie Veneers auch bei starker, bis in das Dentin hinein reichender Präparation zementiert wurden. Dies gilt vor allem für Zähne mit schon bestehenden Füllungen. Hier war die Schmelzsubstanzdicke oft minimal oder im Falle von mesialen und/oder distalen Defekten ganz entfernt worden, was zu einer im Dentin befestigten Restauration führte. Dies könnte eine Erklärung für die vereinzelt notwendigen Rezementierungen sein. Auch andere Studien bestätigen, dass die Notwendigkeit von Rezementierungen bei im Dentin befestigten Veneers steigt (DUMFAHRT und SCHAFFER 2000, FRIEDMAN 1998, PEUMANS et al. 2000). Bemerkenswert ist ferner, dass keines der abgelösten Veneers frakturiert war und somit ohne Komplikationen rezementiert werden konnte. Dies kann durch die stark verbesserten mechanischen Eigenschaften der gepressten Keramiken im Vergleich zur Feldspatkeramik erklärt werden.

Während des gesamten Beobachtungszeitraums verloren zwei von 130 Zähnen (1,5\%) ihre Vitalität und machten so eine endodontische Behandlung notwendig, um den Erhalt des Zahns, aber auch der Funktion zu sichern. Vergleicht man diesen Wert mit denen einer Vollkrone, so ist der Wert als niedrig zu betrachten. Bestätigung findet dies auch in anderen Studien, die von einer niedrigen Rate von devitalisierten bzw. Wurzelkanal zu behandelnden Zähnen während einer Beobachtungszeit zwischen viereinhalb und zehn Jahren berichten (DUMFAHRT und SCHAFFER 2000, MAGNE und DOUGLAS 2000, GUESS und STAPPERT 2008, PIPPIN et al. 1995). Weder im Ober- noch im Unterkiefer kam es bei den eingesetzten Veneers zu Sekundärkaries, was die Aussage früherer Studien bestätigt (DUMFAHRT und SCHAFFER 2000, FRADEANI 1998, PIPPIN et al. 1995, GUESS und STAPPERT 2008, CHEN et al. 2005). Sie zeigen vielmehr, dass das Verlust- bzw. Ereignisrisiko für Veneers in einem Zusammenhang mit der Beschaffenheit der Klebeauflage steht. Danach ist das Risiko für diejenigen Restaurationen erhöht, deren Ränder auf bereits bestehende Füllungen aufgeklebt wurden, während es bei Klebungen auf gesunder Zahnhartsubstanz geringer ist (SHAINI et al. 1997, DUMFAHRT und SCHAFFER 2000). Besonders erwähnenswert ist in diesem Zusammenhang die Untersuchung von PEUMANS et al. (2004). Bei ihr zeigte sich, dass die Veneers, deren Ränder im 
Bereich von Füllungen befestigt wurden, eine Sekundärkariesquote von 10\% nach zehn Jahren Beobachtungszeit aufwiesen.

Alle in der vorliegenden Untersuchung beobachteten Veneers wurden auf gesunde Zahnhartsubstanz fixiert. Es besteht Grund zu der Annahme, dass die richtige Wahl der Lage des Restaurationsrands für das Vorbeugen von Sekundärkaries und somit den Erfolg von Keramikveneers entscheidend ist. Statistisch ließ sich dieser Befund nicht absichern, weil die Anzahl der ausgefallenen Veneers nicht ausreichte, um einen Vergleich zwischen den Ausfällen zu ermöglichen.

Das Ziel dieser Studie war in erster Linie die Beurteilung des Einflusses des Befestigungszements und der Position der Veneers, auf die Überlebens- sowie die Erfolgsrate der Keramikveneers.

In der Literatur findet man nur wenige Studien, die Unterkieferveneers untersucht haben. In den meisten publizierten Arbeiten wurden nur wenige Unterkieferrestaurationen oder gar keine mit einbezogen ((WALTER und RAIGRODSKI 2008, GUESS und STAPPERT 2008, BURKE und LUCAROTTI 2009, LAYTON und WALTON 2007, PEUMANS et al. 2000, MURPHY et al. 2005, AYKOR und OZEL 2009).

In der vorliegenden Untersuchung war die Verteilung der Veneers auf Ober- und Unterkiefer zwar nicht genau gleich, jedoch waren die Verhältnisse mit 41,5\% aller Veneers im Unterkiefer annähernd ausgeglichen. Dies war auch eine Voraussetzung für die statistische Analyse in Form eines Co-Regressions-Modells, zur Beurteilung des Einflusses der Position auf die Überlebens- und Erfolgsraten von Veneerrestaurationen.

Statistisch lassen sich keine Unterschiede zwischen den Versorgungen in beiden Kiefern absichern. Dies gilt sowohl für die Überlebensrate $(p=0,3)$, als auch für die Erfolgsrate $(p=0,4)$. Somit muss davon ausgegangen werden, dass keine Beziehung zwischen Kieferposition und Erfolg bzw. Überleben besteht. Dementsprechend haben Unterkieferversorgungen in Form von Keramikveneers eine ähnliche Prognose wie die Restaurationen im Oberkiefer.

Im Hinblick auf die niedrige Rate an biologischen Komplikationen, in Form von Karies und Vitalitätsverlust, sind Keramikveneers durchaus eine gute Alternative zu Vollkronen, bei Beachtung der jeweiligen Indikationen. Dies gilt auch für den Unterkiefer-Frontzahnbereich. 
Des Weiteren zeigten die statistischen Analysen keinen Einfluss der Wahl des Befestigungszements auf die Überlebens- und Erfolgsrate von Keramikveneers. Daraus lässt sich schließen, dass bei der Wahl eines Etch \& Rinse-Adhäsivsystems die Wahl des Befestigungszements einen zu vernachlässigenden Einfluss auf die klinische Beständigkeit der Restauration hat.

Somit lässt sich die Arbeitshypothese, dass sowohl die Position des Veneers als auch die Wahl des Befestigungszements einen Einfluss auf die Überlebens- und Erfolgswahrscheinlichkeit von gepressten Keramikveneers auf Overlappräparationen haben, nicht bestätigen.

\subsection{Schlussfolgerung}

- Weder in der Verlustrate noch in der Erfolgsquote lässt sich ein signifikanter Einfluss der Kovariablen Region und Zement nachweisen.

- Nach 36 Monaten Beobachtungszeit wird deutlich, dass Frontzahnveneers, die aus Presskeramik gefertigt sind, eine hohe Überlebensrate und eine geringe technische und biologische Fehlerquote zeigen.

- Hauptursache für Komplikationen war die Fraktur der Keramik.

- Im Oberkiefer befestigte Frontzahn-Veneers sind ähnlich erfolgreich, wie die im Unterkiefer zementierten.

- Der Faktor Befestigungszement ist bei klinisch korrekter Anwendung ohne wesentlichen Einfluss auf den Erfolg der Restauration. 


\section{Zusammenfassung}

Die vorliegende Studie nimmt eine retrospektive Bewertung von Frontzahnveneerrestaurationen aus Presskeramik nach 36 Monaten vor. Es wird der Einfluss der Position des Veneers und des Befestigungszements auf den Erfolg bzw. die Überlebensrate untersucht. Sämtliche Behandlungen waren von ein und derselben Person durchgeführt worden.

37 funktionell unauffällige Patienten (weiblich: 21, männlich: 16) im Alter von 20 bis 73 Jahren mit kariesfreiem, suffizient versorgtem Gebiss wurden für die Versorgung mit Veneerrestaurationen in der Ober- und Unterkieferfront (13-23 und 33-43) ausgewählt. Die zu versorgenden Zähne waren füllungsfrei oder wiesen minimale Kompositrestaurationen (max. 2-flächig) auf. Insgesamt wurden 130 Zähne mit Veneerrestaurationen aus Preekeramik Cergo versorgt. Die Verteilung der Veneers war im Oberkiefer ( $n=76): 13: n=10,12: n=13,11: n=16,21: n=17,22: n=14,23: n=6$ und im Unterkiefer $(n=54): 33: n=8,32: n=9,31: n=9,41: n=9,42: n=10,43: n=9$. Die Präparationsform der zu versorgenden Zähne stellte die Overlap-Präparation dar. Als Adhäsiv wurde Optibond FL (Kerr) und als Zement zu 45\% (n=59) Variolink (Ivoclar Vivadent) und zu 55\% (n=71) Calibra (Dentsply) verwendet. Die Patienten wurden in regelmäßigen Abständen einbestellt und es wurden eine zahnärztliche Untersuchung der Veneers mit Sonde, Spiegel und Zahnseide durchgeführt.

Die statistische Auswertung erfolgte zusammen mit der Abteilung Medizinische Statistik der Universitätsmedizin Göttingen (Direktor: Prof. Dr. rer. nat. Brunner).

Die Überlebensrate nach 36 Monaten Tragezeit betrug 97,3\%. Verlustgründe waren vier Frakturen der Keramik und ein biologischer Misserfolg. Negative Ereignisse unter Erhalt der Restauration waren drei Rezementierungen nach Restaurationsverlust sowie zwei endodontische Behandlungen. 95\% waren ereignisfreie Restaurationen.

Es lässt sich festhalten, dass Frontzahnveneerrestaurationen aus Preekeramik Cergo eine hohe Überlebens- und Erfolgsrate nach 36 Monaten Tragezeit aufweisen. Der Befestigungszement spielte bei den vorliegenden klinischen Ergebnissen ebenso keine Rolle wie die Kieferposition des Veneers. 


\section{Literaturverzeichnis}

Andreasen FM, Daugaard-Jensen J, Munksgaard EC (1991): Reinforcement of bonded crown fractured incisors with porcelain veneers. Endod Dent Traumatol $\underline{7}$ : 78-83.

Andreasen FM, Flügge E, Daugaard-Jensen J, Munksgaard EC (1992): Treatment of crown fractured incisors with laminate veneer restorations: an experimental study. Endod Dent Traumatol 8: 30-35.

Aristidis GA, Dimitra B (2002): Five-year clinical performance laminate veneers. Quintessence Int 33:185-9.

Aykor A, Ozel E (2009): Five-year clinical evaluation of 300 teeth restored with porcelain laminate veneers using total-etch and a modified self-etch adhesive system. Oper Dent 2009 34(5):516-23.

Barreiro MM, Riesgo O, Vicento EE (1989): Phase identification in dental porcelains for ceramo-metallic restorations. Dent Mater $\underline{5}$ : 51-57.

Belser UC: Esthetics checklist for the fixed prosthesis. Part II: Biscuit-bake try-in. In: Schärer P., Rinn L. A., Kopp F. R. (eds). Esthetic Guidelines for Restorative Dentistry. Quintessenz, Chicago 1982, 188-192.

Belser U, Magne P, Magne M (1993): Natural and restorative oral esthetics. Part II: Esthetic treatment modalities. J Esthet Dent 5: 239-246.

Belser UC, Magne P, Magne M (1997): Ceramic laminate veneers: continuous evolution of indications. J Esthet Dent 9: 197-207.

Bertolotti RL (1989): Laboratory laminate veneers - a viable alternative. Oral Health; Cosmetic Dent 79(3): 11-16.

Bowen HK : Moderne keramische Werkstoffe. Spektrum Wiss 1986/10: 140-149.

Bowen RL, Chandler HH, Wyckoff HOJ, Misra DN (1978): Metal-filled resin composites II. J Dent Res 57:213-220. 
Boyer DB, Chalkley Y (1982): Bonding between acrylic laminates and composit resin. J Dent Res 61:489-492.

Breustedt A, Lenz E: Stomatologische Werkstoffkunde. Johann Ambrosius Barth Verlag, Leipzig 1978.

Brostow W: Einstieg in die moderne Werkstoffwissenschaft. Hanser, München 1984.

Buonocore MG (1955): A simple method of increasing the adhesion of acrylic filling materials to enamel surfaces. J Dent Res 34: 849.

Burke FJ, Lucarotti PS (2009): Ten-year outcome of porcelain laminate veneers placed within the general dental services in England and Wales. J Dent 37(1):31-8.

Calamia JR (1983): Etched porcelain facial veneers: a new treatment modality based on scientific and clinical evidence. N Y J Dent 53:255-259.

Calamia JR (1985): Etched porcelain veneers: the current state of the art. Quintessence Int $\underline{16}: 5-12$.

Calamia JR (1988): The etched porcelain veneer technique. N Y J Dent 54(7):48-50

Calamia JR (1989): Clinical evaluation of etched porcelain veneer. Am J Dent 2(1): 9-15.

Calamia JR (1993): The current status of etched porcelain veneer restorations. J Indiana Dent Assoc 72:10-15.

Calamia JR, Simonsen RJ (1984): Effect of coupling agents on bond strength of etched porcelain. J Dent Res 63:162-362.

Campbell SD (1989): A comparative strength study of metal ceramic and all-ceramic esthetic materials: Modulus of rupture. J Prosthet Dent 62:476-479.

Castelnuovo J, Tjan AH, Phillips K, Nicholls JI, Kois JC (2000): Fracture load and mode of failure of ceramic veneers with different preparations. J Prosthet Dent 83: $171-180$.

Chan C, Weber $H$ (1986): Plaque retention on teeth restored with full-ceramic crowns: A comparative study. J Prosthet Dent $\underline{\text { 56:666. }}$ 
Chen JH, Shi CX, Wang M, Zhao SJ, Wang H (2005): Clinical evaluation of 546 tetracycline-stained teeth treated with porcelain laminate veneers. J Dent 33(1):3-8.

Christensen GJ, Christensen RP (1991): Clincial observations of porcelain veneers: a three year report. J Esthet Dent $\underline{3}: 174-179$.

Claus $H$ (1980): Werkstoffkundliche Grundlagen der Dentalkeramik. Dent Labor 78:1743-1750.

Claus H (1990): Das Gefüge und Mikrogefüge der Dentalkeramik in Abhängigkeit von den Brennbedingungen. Quintessenz Zahntech 16:1479-1495.

Clyde JS, Gilmour A (1988): Porcelain veneers: a preliminary review. Br Dent J 164:9-14.

Council on Dental materials, instruments and equipment (1984): Statusreport on enamel bonding of composite, performed laminate, and laboratory fabricated resin veneers. J Am Dent Assoc 109(5):762-764.

Cvar J, Ryge G: Criteria for the clinical evaluation of dental restoration materials. (US Public Health Service Publication, No. 790) US: Government Printing Office, San Francisco 1971.

Della Bona A, Kelly JR (2008): The clinical success of all-ceramic restorations. J Am Dent Assoc 139:8-13.

Della Bona A, Kelly JR (2010): A variety of patient factors may influence porcelain veneer survival over a 10-year period. J Evid Based Dent Pract 10(1):35-6.

Della Bona A, Northeast SE (1994): Shear bond strength of resin bonded ceramic after different try-in procedures. J Dent 22:103-107.

Diedrich P: Kieferorthopädie, Ästhetik in der Zahn-, Mund,- Kieferheilkunde, PdZspezial, Urban \& Schwarzenberg, München-Wien-Baltimore1995, 95.

Dumfahrt H, Schaffer H (2000): Porcelain laminate veneers. A retrospective evaluation after 1 to 10 years of service: Part II-Clinical results. Int J Prosthodont 13:9-18. 
Dunne SM, Millar BJ (1993): A longitudinal study of the clinical performance of porcelain veneers. Br Dent J 175:317-321.

Edelhoff D, Özcan $M$ (2007): To what extent does the longevity of fixed dental prostheses depend on the function of the cement? Working Group 4 materials: cementation. Clin Oral Implants Res 18(3):193-204.

Eichner K: Zahnärztliche Werkstoffe und ihre Verarbeitung. Dr. Alfred Hüthig Verlag, Heidelberg 1985.

Evans DB, O'Brien WJ (1999): Fracture strength of glass infiltraded-magnesia core porcelain. Int J Prosthodont 12:38-44.

Faunce FR, Myers DR (1976): Laminate veneer restoration of permanent incisors. J Am Dent Assoc 93:790-792.

Ferrari M, Patroni S, Balleri P (1992): Measurement of enamel thickness in relation to reduction for etched laminate veneers. Int J Periodontics Restorative Dent 23:407-413.

Fradeani M (1998): Six-year follow-up with Empress veneers. Int J Periodontics Restorative Dent 18:216-225.

Fradeani M, Barducci G (1996): Versatility of IPS Empress restorations. Part II: Veneers, inlays, and onlays. J Esthet Dent $\underline{8}: 170-176$.

Freisberg K: Der Einfluß von Feuchtigkeit auf den Elastizitätsmodul und die Biegefestigkeit verschiedener metallkeramischer Massen. Med. Dent. Diss., Köln 1985.

Friedman MJ (1998): A 15-year review of porcelain veneer failure- a clinician's observations. Compend Contin Educ Dent 19:625-628, 630, 632, 638.

Garber DA (1989): Direkte Composite-Verblendung und geätzte Porzellanfacetten. Dental-Report $\underline{4}: 140-144$.

Garber DA, Goldstein RE, Feinman RA: Porcelain laminate veneers. Quintessenz, Chicago 1989. 
Gerds TA, Qvist V, Strub JR, Pipper CB, Scheike TH, Keiding N: Failure time analysis. In: Lesaffre E, Feine J, LeRoux B (eds.): Statistical and methodological aspects of oral health research, John Wiley \& Sons, West Sussex, UK 2009.

Gilmour AJ, Stone DC (1993): Porcelain laminate veneers. a clinical success? Dent Update 20:167-169.

Goldstein RE: Dein neues Lächeln., Quintessenz, Berlin 1990.

Gordan VV, Vargas MA, Denehy GE (1998): Interfacial ultrastructure of the resinenamel region of three adhesive systems. Am J Dent 11:13-16.

Gremminger A: Die Verwendung von Dentalkeramik als Restaurationsmaterial für die Zahnerhaltung im Seitenzahnbereich. Med. Dent. Diss., München 1997.

Guess PC, Stappert CF (2008): Midterm results of a 5-year prospective clinical investigation of extended ceramic veneers. Dent Mater. 24(6):804-13.

Gwinnett AJ (1993): Quantitative contribution of resin infiltration/hybridization to dentin bonding. Am J Dent $\underline{6}: 7-9$.

Hahn P, Gustav M, Hellwig E (2000): An in vitro assessment of the strength of porcelain veneers dependent on tooth preparation. J Oral Rehabil 27:1024-1029.

Helkimo E, Carlsson, GE Helkimo M (1977): Bite force and state of dentition. Acta Odontol Scand 35:297-303.

Highton R, Caputo AA, Matyas J (1987): A photoelastic study of stresses on porcelain laminate preparations. J Prosthet Dent 토:157-161.

Hölsch W, Kappert HF (1992): Festigkeitsprüfungen von vollkeramischem Einzelzahnersatz für den Front- und Seitenzahnbereich. Dtsch Zahnärztl Z 47:621-623.

Holtan JR, Lua MJ, Belvedere PL (1995): Evaluating the effect of glove coating on the shear bonding strength of porcelain laminate veneers. J Am Dent Assoc 126:611616.

Hondrum SO (1992): A review of the strength properties of dental ceramics. J Prosthet Dent 67:859-865. 
Horn HR (1983A): Porcelain laminate veneers bonded to etched enamel. Dent Clin North Am 27:671-684.

Horn HR (1983B): A new lamination: porcelain bonded to enamel. NY Sat Dent J 49:401-403.

Hui KK, Williams B, Davis EH, Holt RD (1991): A comparative assessment of the strengths of porcelain veneers for incisor teeth dependent on their design characteristics. Br Dent J 171:51-55.

Jäger K, Wirz J (1992): Laminates. Quintessenz 43:703-712.

Jäger, K, Stern, M, Wirz, J (1995): Laminates - reif für die Praxis? Quintessenz 46: $1221-1230$.

Jardel V, Degrange M, Picard B, Derrien G (1999): Surface energy of etched ceramic. Int J Prosthodont 12:415-418.

Jordan RF, Boksman I (1984): Conservative vital bleaching treatment of the discolored dentition. Compend Cont Educ Dent $\underline{5}: 803-808$.

Kappert HF, Knode H, Schultheiss $R$ (1991): Festigkeitsverhalten der In-CeramKeramik bei mechanischer und thermischer Wechsellast im Kunstspeichel. Dtsch Zahnärztl Z 46:129-131.

Kelly JR, Nishimura I, Campbell SD (1996): Ceramics in dentistry: Historical roots and current perspectives. J Prosthet Dent 75:18-32.

Kihn PW, Barnes DM (1998): The clinical evaluation of porcelain veneers: a 48month clinical evaluation. J Am Dent Assoc 129:747-752.

Kon M, O`Brien WE, Rasmussen ST, Asaoka K (2001): Mechanical Properties of Glass - only Porcelains Prepared by the Use of Two Feldspatic Frits with Different Thermal Properties. J Dent Res 80:1758-1763.

Kourkouta S, Walsh TT, Davis LG (1994): The effect of porcelain laminate veneers on gingival health and bacterial plaque characteristics. J Clin Periodontol 21: 638640. 
Kreulen CM, Creugers NH, Meijering AC (1998): Meta-analysis of anterior veneer restorations in clinical studies. J Dent 26(4):345-53.

Lampert F: Die Adhäsivtechnik in der Zahnerhaltungskunde. Klinische und experimentelle Untersuchungen zu dem Problem der Schmelzoberflächenverankerung von Füllungsmaterialien. Med. Habilitationsschr., Mainz 1976.

Lampert F, van Gogswaardt DC: Laminierung und Verblendschalen, Ästhetik in der Zahn-, Mund,- Kieferheilkunde, PdZspezial, Urban \& Schwarzenberg, MünchenWien-Baltimore 1995, 166.

Layton D, Walton T (2007): An up to 16-year prospective study of 304 porcelain veneers. Int J Prosthodont 20:389-396.

Levin $\boldsymbol{R}$ (1988): The future of porcelain laminate veneers. Esteth Dent $\underline{1}(2)$ : 45-46.

Lindemann W (2000).: Dentalkeramik - mineralogisch betrachtet. ZMK ㅁ:280-285.

Linden JJ, Swift EJ, Boyer DB, Davis BK (1991): Photo-activation of resin cements through porcelain veneers. J Dent Res $\underline{70}$ :154-157.

Lopes GC, Baratieri LN, de Andrada MA, Vieira LC (2002): Dental adhesion: present state of the art and future. Quintessence Int 33:213-224.

Ludwig D (1991): Untersuchung zur Bruchfestigkeit von vollkeramischen Kronen. Dent Labor 39:647-651.

Luthardt RG, Herold V, Sandkuhl O, Reitz B, Knaak JP, Lenz E (1998): Kronen

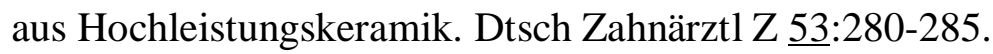

Mackert JR, Williams AL (1996): Microcracks in Dental Porcelain and their Behavior during Multiple Firing. J Dent Res 포:1484-1490.

Magne P, Belser U: Bonded porcelain restorations in the anterior dentition. A biomimetic approach. Quintessenz, Chicago 2002.

Magne P, Belser $\boldsymbol{U}$ : Adhäsiv befestigte Keramikrestaurationen - Biomimetische Sanierungen im Frontzahnbereich. Quintessenz Bibliothek, Berlin 2004, 58, 130, 164-166. 
Magne P, Douglas WH (1999A): Additive contour of porcelain veneers: A key element in enamel preservation, adhesion and esthetic for the aging dentition. J Adhes Dent 1:81-91.

Magne P, Douglas WH (1999B): Optimisation des concepts mécanique en medicine

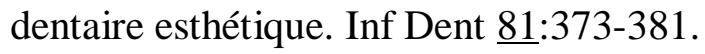

Magne P, Douglas WH (1999C): Design optimization and evolution of bonded ceramics for the anterior dentition: a finite-element analysis. Quintessence Int 30:661672.

Magne P, Douglas WH (1999D): Porcelain veneers: dentin bonding optimization and biomimetic recovery of the crown. Int J Prosthodont 12:111-121.

Magne P, Douglas WH (2000): Interdental design of porcelain veneers in the presence of composite fillings: finite element analysis of composite shrinkage and thermal stresses. Int J Prosthodont 13(2):117-24.

Magne P, Holz J (1994): Restauration des dents antérieures. Principe, indications et limites des techniques de traitement. Rev Mens Suisse Odontostomatol 104:12471254.

Magne P, Magne M, Belser U (1993): Natural and oral esthetics. PartI: Rationale and basic strategies for successful esthetic rehabilotations. J Esthet Dent $\underline{5}: 161-173$.

Magne P, Magne M, Belser U (1996): The diagnostic template: Key element of a comprehensive esthetic treatment concept. Int J Periodontics Restorative Dent 16(6):561-569.

Magne P, Kwon KR, Belser UC, Hodges JS, Douglas WH (1999): Crack propensity of porcelain laminate veneers: A simulated operatory evaluation. J Prosthet Dent 81:327-334.

Magne P, Perroud R, Hodges JS, Belser UC (2000): Clinical performance of noveldesign porcelain veneers for the recovery of coronal volume and length. Int J Periodontics Restorative Dent 20:441-457.

Marx R (1993): Moderne keramische Werkstoffe für ästhetische RestaurationenVerstärkung und Bruchzähigkeit. Dtsch Zahnärztl Z 4ㄹ:229-236. 
Masuhara E (1969): Über die Chemie eines neuen haftfähigen Kusstofffüllungsmaterials. Dtsch Zahnärztl Z 24:620.

McLaughlin G (1988): Porcelain fused to tooth - the state of the art. Rest Dent 4(4):90-94.

Meijering AC, Roeters FJ, Mulder J, Creugers NH (1997): Patients' satisfaction with different types of veneer restorations. J Dent 25:493-497

Meijering AC, Creugers NH, Roeters FJ (1998): Survival of three types of veneer restoration in a clinical trial: a 2.5-year interim evaluation. J Dent 26:563-568.

Morena R, Lockwood PE, Fairhurst CW (1986): Fracture toughness of commercial dental porcelains. Dent Mater 2:58-62.

Mouradian WF, Graham D, Fernald L (1976): A new approach to treatment of tetracycline stained teeth:Report of a case. J Dent Child 43:103.

Mouradian WF, Graham D, Fernald L (1978): A new approach to treating tetracycline stained teeth: a follow-up report. J Dent Child $\underline{45}: 473$.

Murphy E, Ziada HM, Allen PF (2005): Retrospective study on the performance of porcelain laminate veneers delivered by undergraduate dental students. Eur J Prosthodont Restorative Dent 13:38-43.

Nattress BR, Youngson CC, Patterson C, Martin DM, Ralph JP (1995): An in vitro assessment of tooth preparation for procelain veneer restorations. J Dent 23:165-170.

Nicholls JI (1988): Tensile bond of resin cements to porcelain veneers. J Prosthet Dent 60:443-447.

Nixon RL: Porzellan-Verblendungen, eine ästhetische Möglichkeit der Behandlung. In: Rufenacht, C.: Ästhetik in der Zahnheilkunde, Quintessenz, Berlin 1990, 329368.

Nordbo H, Rygh-Thoresen N, Henaug T (1994): Clinical performance of porcelain laminate veneers without incisal overlapping: 3-year results. J Dent 22:342-345. 
Palmer DS, Barco MT, Billy EJ (1992): Temperature extremes produced orally by hot and cold liquids. J Prosthet Dent 67:325-327.

Pape FW, Kerschbaum T (1992): Veneers - ein Überblick. Zahnärztl Mitt 2:18-24.

Permann R, Kuderna F, Parsche E, Moser F, Bratschko RO, Riedl R (1989): Die geklebte Porzellanschale - Eine Alternative zur Porzellanfrontzahnkrone, Z Stomatol 86:197-206.

Peumans M, Van Meerbeek B, Lambrechts P, Vuylsteke-Wauters M, Vanherle G (1998): Five-year clinical performance of porcelain veneers. Quintessence Int 29:211-221.

Peumans M, Van Meerbeek B, Yoshida Y, Lambrechts P, Vanherle G (1999): Porcelain veneers bonded to tooth structure: an ultra-morphological FE-SEM examination of the adhesive interface. Dent Mater 15:105-119.

Peumans M, Van Meerbeek B, Lambrechts P, Vanherle G (2000): Porcelain veneers: a review of the literature. J Dent 28:163-177.

Peumans M, De Munck J, Fieuws S, Lambrechts P, Vanherle G, Van Meerbeek $\boldsymbol{B A}$ (2004): prospective ten-year clinical trial of porcelain veneers. J Adhesive Dent $\underline{6}: 65-76$.

Pincus CR (1938): Building mouth personality. J South California Dent Ass 14:125129.

Pippin DJ, Mixson JM, Soldan-EIs AP (1995): Clinical evaluation of restored maxillary incisors: veneers vs. PFM crowns. J Am Dent Assoc 126(11):1523-9.

Poß P: Biege- und Spaltzugfestigkeitsprüfungen metall-keramischer Massen in Abhängigkeit von der Anzahl der Vakuumbrände. Med. Dent. Diss., Köln 1983.

Pröbster L: Klinische Erfahrung mit vollkeramischem Zahnersatz - Ein Rückblick. In: Vollkeramik: Werkstoffkunde - Zahntechnik - Klinische Erfahrung. HF Kappert editor. Quintessenz, Berlin 1996, 103-116.

Ritter JE (1995): Predicting lifetimes of materials and material structures. Dent Mater 11:142-146. 
Rochette AL (1975): A ceramic restoration bonded by etched enamel and resin for fractured incisors. J Prosthet Dent 33:287-293.

Rosenblum MA, Schulman A (1997): A Review of All-Ceramic Restorations. J Am Dent Assoc 128:297-307.

Roulet JF, Söderholm KJM, Longmate $J$ (1995): Effects of treatment and storage conditions on ceramic/ composite bond strength. J Dent Res 74:381-387.

Rucker LM, Richter W, MacEntee M, Richardson A (1990): Porcelain and resin veneers clinically evaluated: 2-year results. J Am Dent Assoc 121:594-596.

Salmang H, Scholze H: Keramik, Teil 1 und 2. Springer-Verlag Berlin, Heidelberg 1983.

Schmalz G, Geurtsen W, Haller B, Federlin M (2007): Zahnfarbene Restaurationen aus Keramik: Inlays, Teilkronen und Veneers , Gemeinsame Stellungnahme der Deutschen Gesellschaft für Zahnerhaltung/DGZ und der Deutschen Gesellschaft für Zahn-, Mund- und Kieferheilkunde/DGZMKm (September 2007).

Schmid M, Fischer J, Salk M, Strub J (1992): Mikrogefüge Leuzit-verstärkter Glaskeramiken. Schweiz Monatsschr Zahnmed 102:1046-1052.

Schnapp JD, Köhler G: Technische Keramiken - eine Übersicht. In: Bearbeitung, Fügen und Prüfen von Keramik. DVS Verlag, Jena 1990.

Schneider PM, Messerm LB, Douglas WH (1981): The effect of enamel reduction in vitro on the bonding of composite resin to permanent human enamel. J Dent Res 60:895-900.

Schultheiss R: Biegefestigkeit verschiedener Dentalkeramiken nach Thermolastwechsel und Lagerung in Kunstspeichel. Med. Dent. Diss., Freiburg 1992.

Shaini FJ, Shortall AC, Marquis PM (1997): Clinical performance of porcelain laminate veneers. A retrospective evaluation over a period of 6.5 years. J Oral Rehabil 24:553-559.

Sheets CG, Taniguchi T (1990): Advantages and limitations in the use of porcelain veneer restorations. J Prosthet Dent 64:406-411. 
Sheth J, Jensen M, Tolliver D (1988): Effect of surface treatment on etched porcelain bond strength to enamel. Dent Mater 4:328-337.

Sim C, Neo J, Chua EK, Tan BY (1994): The effect of dentin bonding agents on the microleakage of porcelain veneers. Dent Mater 10:278-281.

Simonsen E, Calamia JR (1983): Tensile bond strength of etched porcelain. J Dent Res Abstr 1154, 62:297.

Sonnenburg M, Fethke K, Riedel S, Voelker H (1978): The load carrying capacity of the teeth of the human jaw. Zahn Mund Kieferheilkd Zentralbl 66:125-132.

Stacey GD (1993): A shear stress analysis of the bonding of porcelain veneers to enamel. J Prosthet Dent 70:395-402.

Stangel I, Nathanson D, Hsu CS (1987): Shear strength of the composite bond to etched porcelain. J Dent Res $\underline{66}: 1460-1465$.

Stokes AN, Hood JAA (1993): Impact fracture characteristics of intact crown human central incisors. J Oral Rehabil 20:89-95.

Strassler HE, Nathanson D (1989): Clinical evaluation of etched porcelain veneers over a period of 18 to 42 months. J Esthet Dent $\underline{1}$ :21-28.

Strassler HE, Weiner S (1995): Seven to ten year clinical evaluation of etched porcelain veneers. J Dent Res 74:176.

Strub JR (1992): Vollkeramische Systeme. Dtsch Zahnärztl Z 47:566-571.

Swift EJ Jr, Friedman MJ (2006): Critical appraisal. Porcelain veneer outcomes, part I. J Esthet Restor Dent 18(1):54-7.

Swift EJ Jr., Perdigao J, Heymann HO (1995): Bonding to enamel and dentin: a brief history and state of the art. Quintessence Int 26:95-110.

Tietz HD: Technische Keramik. VDI-Verlag, Düsseldorf 1994.

Touati B, Bersay L (1987): Enameling of teeth using glass ceramic veneers. Cah Prothese 15:167-189. 
Touati B, Pissis P, Miara P (1985): Bonded single restorations and the concept of pellicular preparations. Cah Prothese 13:95-130.

Touati B, Plissart-Vanackere A (1990): Ceramic bonded veneers. Toward a minimal prosthesis. Real Clin 1: $51-66$

Troedson M, Derand T (1998): Shear stresses in the adhesive layer under porcelain veneers. A finite element method study. Acta Odontol Scand 56:257-262.

Troedson M, Derand T (1999): Effect of margin design, cement polymerization, and angle of loading on stress in porcelain veneers. J Prosthet Dent 82:518-524.

Van der Zel J (1988): Werkstoffkundliche Aspekte für die Porzellan-Inlay/OnlayTechnik. Dent Labor 36:865.

Van Gogswaardt DC (1989): Schichtkeramikfacetten zur kosmetischen Frontzahn-

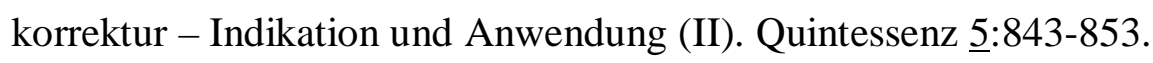

Van Thoor W: Klinische Standfestigkeit keramischer Veneers, eine 7jährige Retrospektive, Shaker-Verlag Aachen 1998, 31.

Wall JG, Reisbick MH, Espeleta KG (1992): Cement luting thickness beneath porcelain veneers made on platinum foil. J Prosthet Dent 68:448-450.

Walls AW (1995A): The use of adhesively retained al-porcelain veneers during the management of fractured and worn anterior teeth: Part I. Clinical technique. Br Dent $\mathrm{J} \underline{178}$ :333-336.

Walls AW (1995B): The use of adhesively retained al-porcelain veneers during the management of fractured and worn anterior teeth: Part II. Clinical results after 5 years of follow-up. Br Dent J 178:337-340.

Walls AW, Steele JG, Wassell RW (2002): Crowns and other extra-coronal restorations: porcelain laminate veneers. Br Dent J 193:73-76, 79-82.

Walter RD, Raigrodski AJ (2008): Critical appraisal: clinical considerations for restoring mandibular incisors with porcelain laminate veneers. J Esthet Restor Dent 20(4):276-81. 
Wang Y, Raffaelli O, Zhang L, Chen J, Ferrari M (2007): Effect of different bonding procedures on micro-tensile bond strength between a fiber post and resin-based luting agents. J Oral Sci $\underline{49}(2): 155-60$.

Watanabe I, Nakabayashi N, Pashley DH (1994): Bonding to ground dentin by a phenyl-P self-etching primer. J Dent Res $\underline{73}$ :1212-1220.

Yilmaz H, Aydin C, Gul BE (2007): Flexural strength and fracture toughness of den-

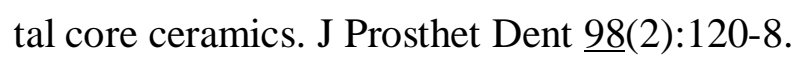

Yoshiyama M, Matsuo T, Ebisu S, Pashley D (1998): Regional bond strength of self-etching/ self-priming adhesive systems. J Dent 26:609-610. 


\section{Anhang}

\subsection{USPHS-Kriterien}

Tab. 16: Bewertung der Veneers anhand der Ryge-USPHS-Kriterien (CVAR und RYGE 1971)

\begin{tabular}{|c|c|c|}
\hline Characteristics & Rating & Criteria \\
\hline \multirow{2}{*}{$\begin{array}{l}\text { Secondary } \\
\text { caries }\end{array}$} & Alpha & No evidence of caries contiguous with the margin of the restoration \\
\hline & Bravo & Caries evident contiguous with the margin of the restoration \\
\hline \multirow{3}{*}{$\begin{array}{l}\text { Marginal } \\
\text { adaptation }\end{array}$} & Alpha & $\begin{array}{l}\text { No visible evidence of crevice along margin; no catch or penetration of } \\
\text { explorer }\end{array}$ \\
\hline & Bravo & $\begin{array}{l}\text { Visible evidence of crevice and/or catch of explorer; no penetration of } \\
\text { explorer }\end{array}$ \\
\hline & Charlie & Visible evidence of crevice; penetration of explorer \\
\hline \multirow{3}{*}{$\begin{array}{c}\text { Marginal } \\
\text { discoloration }\end{array}$} & Alpha & $\begin{array}{l}\text { No discoloration on the margin between the restoration and the tooth } \\
\text { structure }\end{array}$ \\
\hline & Bravo & $\begin{array}{l}\text { Superficial discoloration on the margin between the restoration and the } \\
\text { tooth structure; does not penetrate in pulpal direction }\end{array}$ \\
\hline & Charlie & $\begin{array}{l}\text { Discoloration has penetrated along the margin of the restorative } \\
\text { material in pulpal direction }\end{array}$ \\
\hline \multirow{3}{*}{ Color match } & Alpha & $\begin{array}{l}\text { No mismatch in color, shade and/or translucency between restoration } \\
\text { and adjacent tooth }\end{array}$ \\
\hline & Bravo & $\begin{array}{l}\text { Mismatch between restoration and tooth structure within the normal } \\
\text { range of color, shade and/or translucency ( }<1 \text { shade off; Vita shade } \\
\text { guide) }\end{array}$ \\
\hline & Charlie & $\begin{array}{l}\text { Mismatch between restoration and tooth structure outside the normal } \\
\text { range of color, shade and/or translucency ( }>1 \text { shade off; Vita shade } \\
\text { guide) }\end{array}$ \\
\hline \multirow{3}{*}{ Anatomic form } & Alpha & The restoration is continuous with tooth anatomy \\
\hline & Bravo & $\begin{array}{l}\text { The restoration is not continuous with tooth anatomy. The restoration } \\
\text { is slightly under- or over-contoured }\end{array}$ \\
\hline & Charlie & $\begin{array}{l}\text { The restoration is not continuous with tooth anatomy. Restoration } \\
\text { material is missing; a surface concavity is ascertainable }\end{array}$ \\
\hline
\end{tabular}




\subsection{Genehmigung des Ethikantrags}

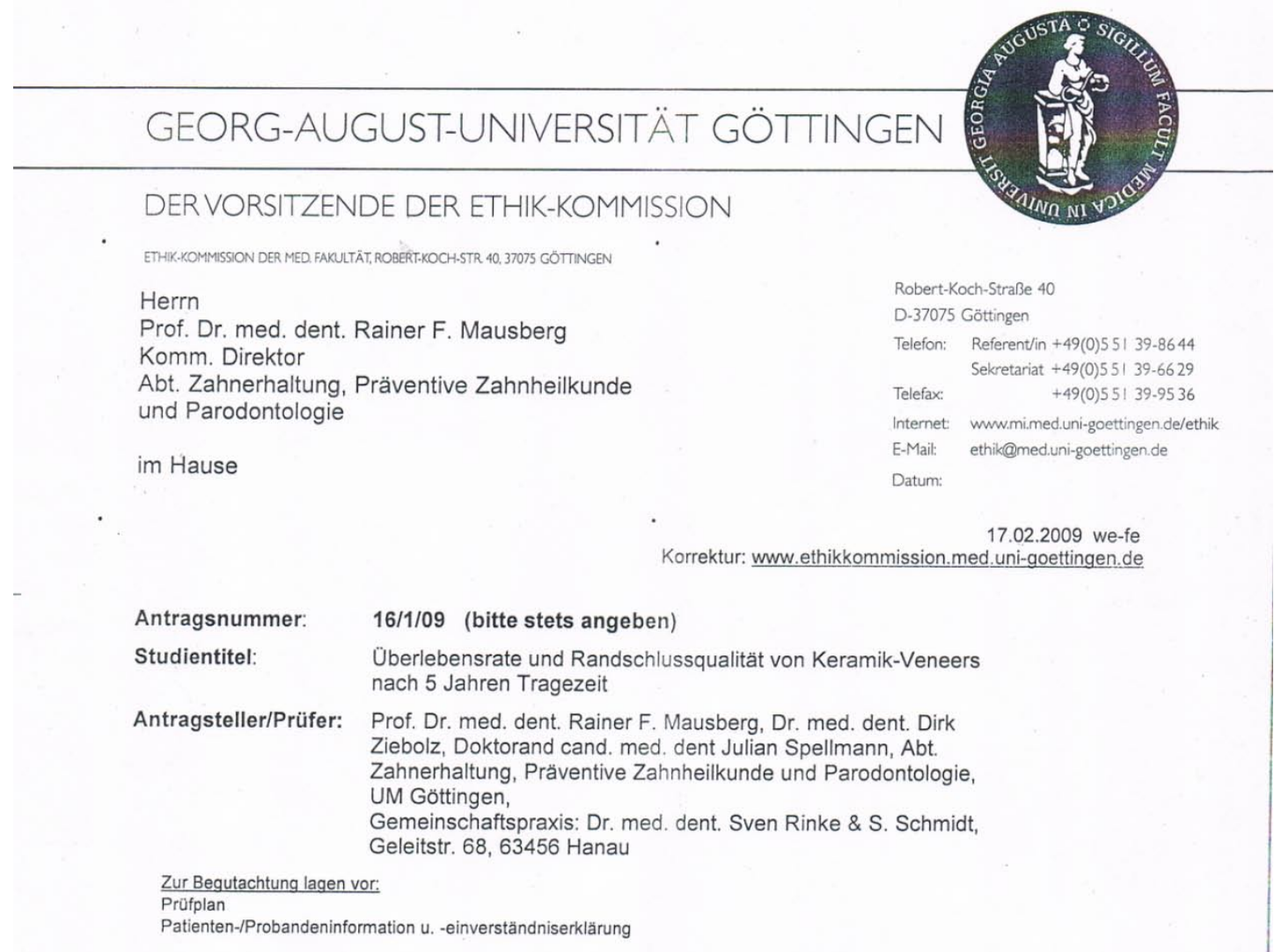

Sehr geehrter Herr Kollege Mausberg,

wir bestätigen den Eingang Ihres Schreibens vom 14.02.2009 am 16.02.2009.

Nach Ergänzung der vorliegenden Dokumente (folgende Unterlagen wurden vorgelegt: geändertes Studienprotokoll, geänderte Patienteninformation) und Beantwortung der im vorläufigen Votum aufgeführten Fragen bestehen nunmehr keine ethischen und rechtlichen Bedenken gegen die Durchführung des oben genannten Forschungsvorhabens.

Wir wünschen viel Erfolg bei der Durchführung Ihres Projektes.

Die Ethik-Kommission weist darauf hin, dass die ärztliche und juristische Verantwortung bei den jeweiligen Prüfärzten verbleibt. Auf die Einhaltung einschlägiger Gesetze und Rechtsvorschriften wird hingewiesen. Die nach Rechtslage notwendigen Unterrichtungen ( $u$. A. Prüfplanänderungen, entsprechende Zwischenfallsereignisse, neue Datenlage, Nachmeldung von Prüfzentren, Abschlussbericht) sind der Ethik-Kommission unverzüglich vorzulegen. Die EthikKommission bestätigt, dass sie auf Grundlage nationaler Gesetze, Vorschriften sowie der GCP/ICH-Richtlinie arbeitet.

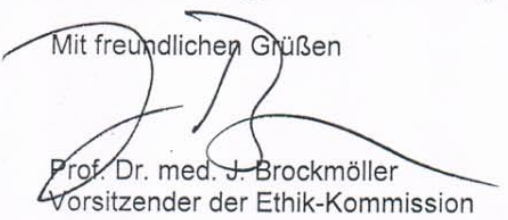




\subsection{Untersuchungsbogen}

Studie:

„Erfolgsrate/Beständigkeit von Keramik-Veneers nach 5 Jahren Tragezeit“

\section{Studien-Nummer:}

\section{Untersucher:}

Patient LFD-Nr.Patienteninitialen:

Geschlecht:

$\square$ männlich $\square$ weiblich

Geburtsdatum:

$$
\text { t t m m j j }
$$

Leiter der Studie:

Prof. Dr. med. dent. Rainer F. Mausberg

Universitätsklinikum Göttingen

Zentrum der Zahn- Mund- und Kieferheilkunde,

Zahnerhaltung, Präventive Zahnheilkunde und Parodontologie

Robert-Koch-Straße 40

37075 Göttingen

Tel.: 0551-39-2878

Fax: $39-8368$

Email: rainer.mausberg@med.uni-goettingen.de

Ort

Datum

Unterschrift des Untersuchers 
KLINISCHE BEFUNDE

Datum:_______

Pat. \#:

Patienteninitialen:

\begin{tabular}{|c|c|c|c|c|c|c|c|}
\hline \multirow[t]{2}{*}{ Veneer } & \multirow{2}{*}{$\begin{array}{l}\text { Zem-Dat. } \\
\text { Datum }\end{array}$} & $\begin{array}{c}\text { Befestigungs- } \\
\text { zement }\end{array}$ & $\begin{array}{c}\text { Kontroll- } \\
\text { Dat. }\end{array}$ & \multicolumn{2}{|c|}{ in-situ } & \multirow{2}{*}{$\begin{array}{l}\text { Verlust-Dat. } \\
\text { Datum }\end{array}$} & \multirow{2}{*}{$\begin{array}{c}\text { Intervenierungs- } \\
\text { Dat. } \\
\text { Datum }\end{array}$} \\
\hline & & & Datum & ja & nein & & \\
\hline 13 & & & & & & & \\
\hline 12 & & & & & & & \\
\hline 11 & & & & & & & \\
\hline 21 & & & & & & & \\
\hline 22 & & & & & & & \\
\hline 23 & & & & & & & \\
\hline 33 & & & & & & & \\
\hline 32 & & & & & & & \\
\hline 31 & & & & & & & \\
\hline 41 & & & & & & & \\
\hline 42 & & & & & & & \\
\hline 43 & & & & & & & \\
\hline
\end{tabular}


Bei Verlust: Verlustursache

\begin{tabular}{|c|c|c|c|c|c|c|c|c|c|c|}
\hline \multirow[t]{3}{*}{ Veneer } & \multicolumn{4}{|c|}{ Fraktur } & \multicolumn{6}{|c|}{ Biologische Gründe (Extraktion) } \\
\hline & \multicolumn{2}{|c|}{ In d. Keramik } & \multicolumn{2}{|c|}{ an d. Grenzfläche } & \multicolumn{2}{|c|}{ Karies } & \multicolumn{2}{|c|}{ PAR } & \multicolumn{2}{|c|}{ Endo } \\
\hline & ja & nein & ja & nein & ja & nein & ja & Nein & ja & nein \\
\hline 13 & & & & & & & & & & \\
\hline 12 & & & & & & & & & & \\
\hline 11 & & & & & & & & & & \\
\hline 21 & & & & & & & & & & \\
\hline 22 & & & & & & & & & & \\
\hline 23 & & & & & & & & & & \\
\hline 33 & & & & & & & & & & \\
\hline 32 & & & & & & & & & & \\
\hline 31 & & & & & & & & & & \\
\hline 41 & & & & & & & & & & \\
\hline 42 & & & & & & & & & & \\
\hline 43 & & & & & & & & & & \\
\hline
\end{tabular}


Bei Intervention: Ursache/Art der Intervention

\begin{tabular}{|c|c|c|c|c|c|c|c|c|c|c|}
\hline \multirow[t]{2}{*}{ Veneer } & \multicolumn{2}{|c|}{ Randverfärbung } & \multicolumn{2}{|c|}{ Rezementierung } & \multicolumn{2}{|c|}{$\begin{array}{l}\text { Randschluss } \\
\text { insuffizient }\end{array}$} & \multicolumn{2}{|c|}{ Wurzelbehandlung } & \multicolumn{2}{|c|}{ Sekundärkaries } \\
\hline & ja & nein & ja & nein & ja & nein & ja & nein & ja & nein \\
\hline 13 & & & & & & & & & & \\
\hline 12 & & & & & & & & & & \\
\hline 11 & & & & & & & & & & \\
\hline 21 & & & & & & & & & & \\
\hline 22 & & & & & & & & & & \\
\hline 23 & & & & & & & & & & \\
\hline 33 & & & & & & & & & & \\
\hline 32 & & & & & & & & & & \\
\hline 31 & & & & & & & & & & \\
\hline 41 & & & & & & & & & & \\
\hline 42 & & & & & & & & & & \\
\hline 43 & & & & & & & & & & \\
\hline
\end{tabular}

Ort 


\section{Danksagung}

Hiermit möchte ich mich bei allen Beteiligten bedanken, die dazu beigetragen haben, dass meine Arbeit in dieser Form abgeschlossen werden konnte.

In erster Linie möchte ich meinem Doktorvater Prof. Dr. med. dent. Rainer F. Mausberg danken mir dieses Thema überlassen zu haben. Ebenso möchte ich Dr. med. dent. Dirk Ziebolz für die ständige Betreuung und die zahlreichen Anregungen und Verbesserungsvorschläge danken. Außerdem danke ich Dr. Rinke und seinem gesamten Team für die Hilfe bei der Umsetzung des praktischen Teils.

Für die Zusammenstellung und Auswertung der statistischen Daten möchte ich mich neben den Betreuern in der Statistik-Sprechstunde vor allem bei Katharina Lange bedanken. 


\section{Lebenslauf}

Am 17.09.1984 wurde ich als zweites Kind meiner Eltern, Prof. Dr. Hermann Spellmann und Dr. Genoveffa Spellmann, geb. Renna, in Göttingen geboren. Von 1991 bis 1995 besuchte ich die katholische Godehard-Grundschule in Göttingen. Darauf folgten zwei Jahre an der katholischen Bonifatiusschule. Von 1997 bis 2004 war ich Schüler am Felix-Klein-Gymnasium in Göttingen, das ich mit Erlangen der allgemeinen Hochschulreife abschloss.

Am 01.07.2004 trat ich meinen Zivildienst im evangelischen Krankenhaus Weende an.

Zum Sommersemester 2005 nahm ich mein Studium der Zahnheilkunde in Göttingen auf. Die naturwissenschaftliche Vorprüfung absolvierte ich im März 2006. Im September 2007 legte ich die zahnärztliche Vorprüfung ab. Im Juli 2010 schloss ich das Staatsexamen der Zahnmedizin ab.

Meine Approbation als Zahnarzt erhielt ich am 05.07.2010.

Seit dem 01.11.2010 arbeite ich als Vorbereitungsassistent in der Zahnarztpraxis Dres. Brauner in Dessau-Rosslau. 